\title{
DE91 DO058I
}

\section{AFDM:}

An Adoanced Fluid-Dymamics Model

Volume II: Topologies, Flow Regimes, and Interfacial Areas

D. Wilhelm*

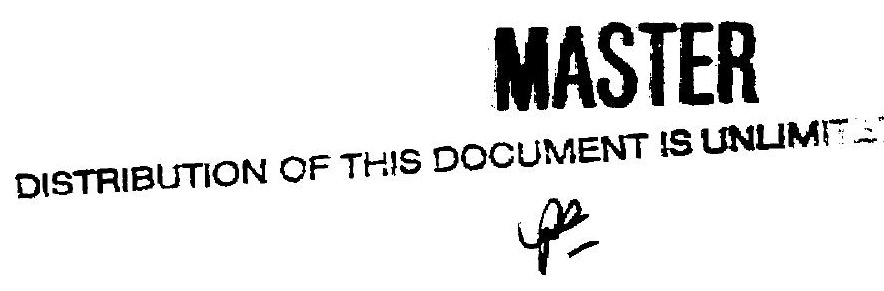


ABSTRACT ............................... vi

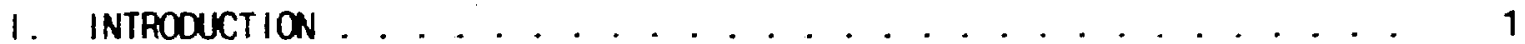

11. CONTIMUOUS PHASE AND TOPOLOGY . . . . . . . . . . . . . . . . . . 5

A. Cont inuous Phase . . . . . . . . . . . . . . . . . . . . . . 5

B. Continuous Liquid. . . . . . . . . . . . . . . . . . . . . . 13

C. Topology . . . . . . . . . . . . . . . . 13

D. Flow Regimes in Unrestricted Two-Phase Flow. . . . . . . . . . 15

1. Nucleat ing Regime. . . . . . . . . . . . . . . . . . 16

2. Coalescing Regime.................... . 16

3. Churn-Turbulent Regime ................. 19

4. Cellular Regime................. . . . 20

5. Droplet Regime . . . . . . . . . . . . . . . 20

III. SURFACE AREA SOURCE TERM AND UPDATE . . . . . . . . . . . . . 21

A. Update Due to Hydrodynamic Models . . . . . . . . . . . . . . . 22

B. Surface Area Source Terms . . . . . . . . . . . . . . . . . . . 26

1. Bubble Nucleation ................ . . 27

2. Dynanic Forces for Bubbles . . . . . . . . . . . . . . . 20

3. Dynanic Forces for Droplets .................. . 31

4. Buoyancy-Driven Turbulence Breakup . . . . . . . . . . . . 34

5. Flashing of Droplets .................. 38

6. Coalescence..................... 40

C. Special Cases ........... . . . . . . . . . . . 42

D. Update Due to Mass Transfer ... . . . . . . . . . . . . 43

IV. SURFACE AREA SUBDIVISION ...................... . . . . 15

A. Structure Contact . . . . . . . . . . . . . . . . . . . . 46

B. Liquid 1 - Solid Particulate Contact . . . . . . . . . . 47

C. Collision Contact................... 54

D. Persisting Contact . . . . . . . . . . . . . . 55

E. Combination of Persisting and Collision Contact . . . . . . . 60

F. Correction Due to Nucleation . . . . . . . . . . . . . . . 61

G. Surface Area Mltiplier . . . . . . . . . . . . . . . . . . 62

H. Interface Temperature . . . . . . . . . . . . . . . . 63

K. Subdivisions .................. . . 63

1. Topology 2 Subdivision ................ 64

2. Topology 4 and 7 Subdivision .............. . 64

3. Topology 5 and 6 Subdivision . . . . . . . . . . . . 65

4. Topology 8 and 9 Subdivision ............. . . 67

5. Topology 10 Subdivision . . . . . . . . . . . . . 68

6. Topology 11 Subdivision ................ . 72

7. Topology 12 Subdivision . . . . . . . . . . . . . . 75 
v. SURFACE AREA CONVECTION ................ 82

A. Area Convection Model 1............... 82

B. Area Convection Model 2................ 86

1. Outflow Terms ................. 87

2. Inflow Terms ................... 89

VI. INITIAL CONDITIONS AND USER INPUT ................ 91

A. Initial Conditions . . . . . . . . . . . . . . . . . 91

B. User Input ................... 92

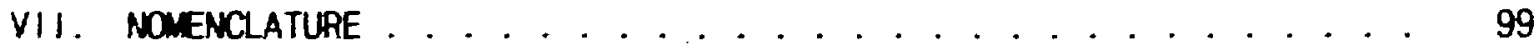

REFERENCES . . . . . . . . . . . . . . . . 122 


\begin{abstract}
MBSTRACT
This volume describes the Advanced Fluid-Dynamics Model (AFDM) for topologies, flow regimes, and interfacial areas. The objective of these models is to provide values for the interfacial areas between all components existing in a computational cell. The interfacial areas are then used to evaluate the mass, energy, and momentum transfer between the components. A new approach has been undertaken in the development of a model to convect the interfacial areas of the discontinuous velocity fields in the three-velocity-field enviromment of AFDM. These interfacial areas are called convectible surface areas. The continuous and discontinuous components are chosen using volume fraction and levitation criteria. This establishes socalled topologies for wich the convectible surface areas can be determined. These areas are functions of space and time. Sol id particulates that are limited to being discont inuous within the bulk fluid are assumed to have a constant size. The convectible surface areas are subdivided to model contacts between two discontinuous components or discont inuous components and the structure. The models have been written for the flow inside of large pools. Therefore, the structure is tracked only as a boundary to the fluid volume without having a direct influence on velocity or volume fraction distribution by means of flow regimes or boundary layer models. This volume refers to AFDM Vols. I, III, VI, and VII.
\end{abstract}




\title{
AFD: N AONAMCE FLUID-DMNMICS MODEL \\ Volume 11: Topologies, Flow Regiwes, and interfacial Areas
}

\author{
by \\ D. Wilhelm \\ Contributors
}

J. Berthier, W. R. Bohl, S. Kondo, and F. Ruel

\section{InTmonction}

The objective of this volume is to provide the values of all interfacial areas in a given Advanced Fluid-dynamics Model (AFDM) computational cell. These values will subsequently be used in calculating the momentum-exchange coefficients and the heat and mass transfer.

In Fig. 1, the relevant parts of the code are listed together with a short description of the tasks. Eventually, the calculation of the instantaneous interfacial areas at the end of subroutine INIIFA is the final step for the calculational procedure. These interfacial areas are then used in the center box of Step 1 in Fig. 1. All other tasks in the rest of the boxes provide the necessary data to calculate the instantaneous interfacial areas. In three of the boxes, there are two parallel vertical dotted lines of the two compile options, ARCV1 and ARCV2, describing the area convection model 1 and model 2, respectively. A detailed description will be given in Sec. $V$. Here, we concentrate on the main objectives to outline the computational procedure. The instantaneous interfacial areas are subdivisions of the convectible surface areas that describe the exclusive contact between the discontinuous velocity fields and the cont inuous velocity field.

For component 1 , the solid particulates are not accounted for because they are assumed to have a constant radius. The convectible surface areas oo not 


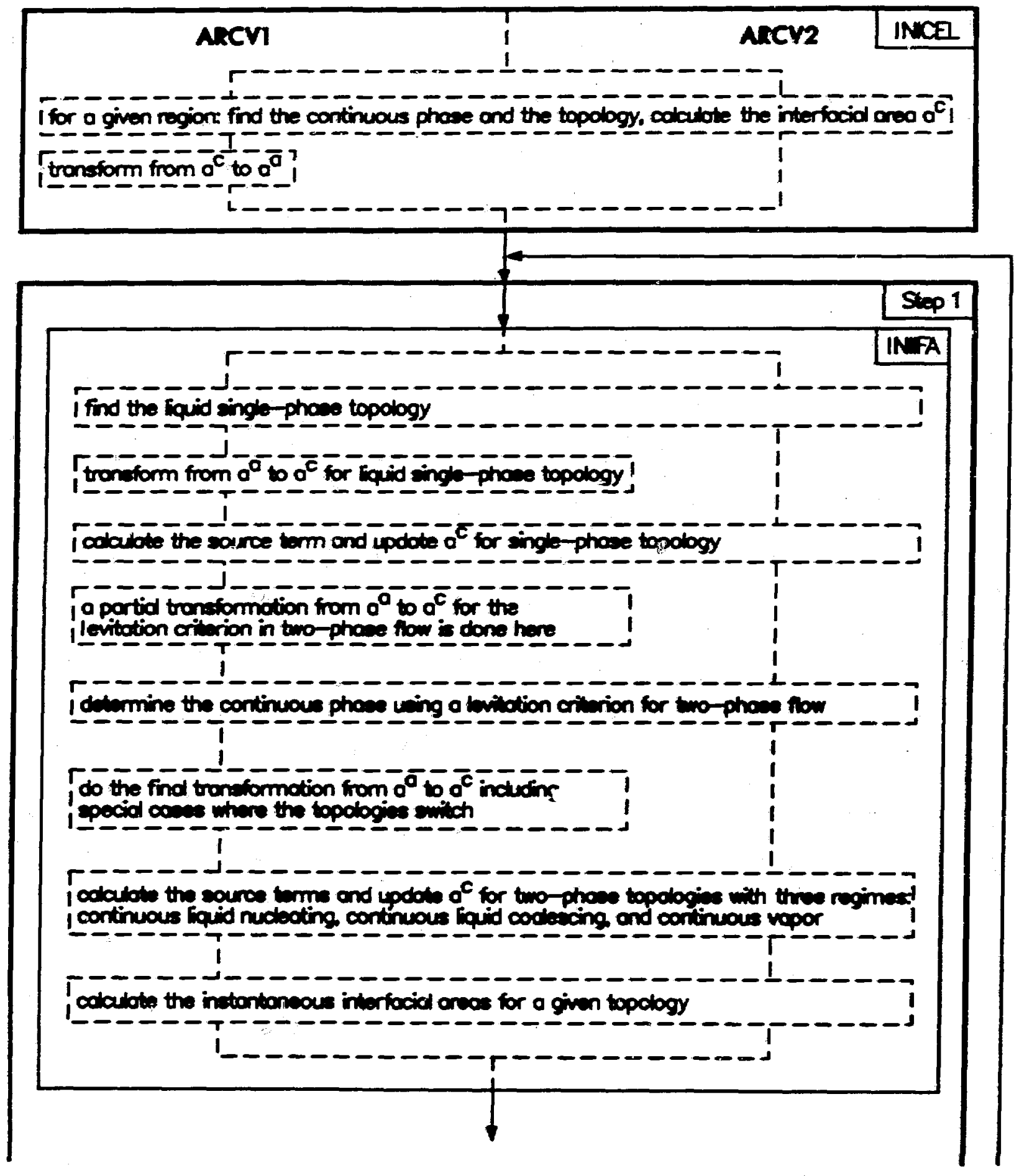

Fig. 1 .

AFDM computational flow for interfacial areas. 


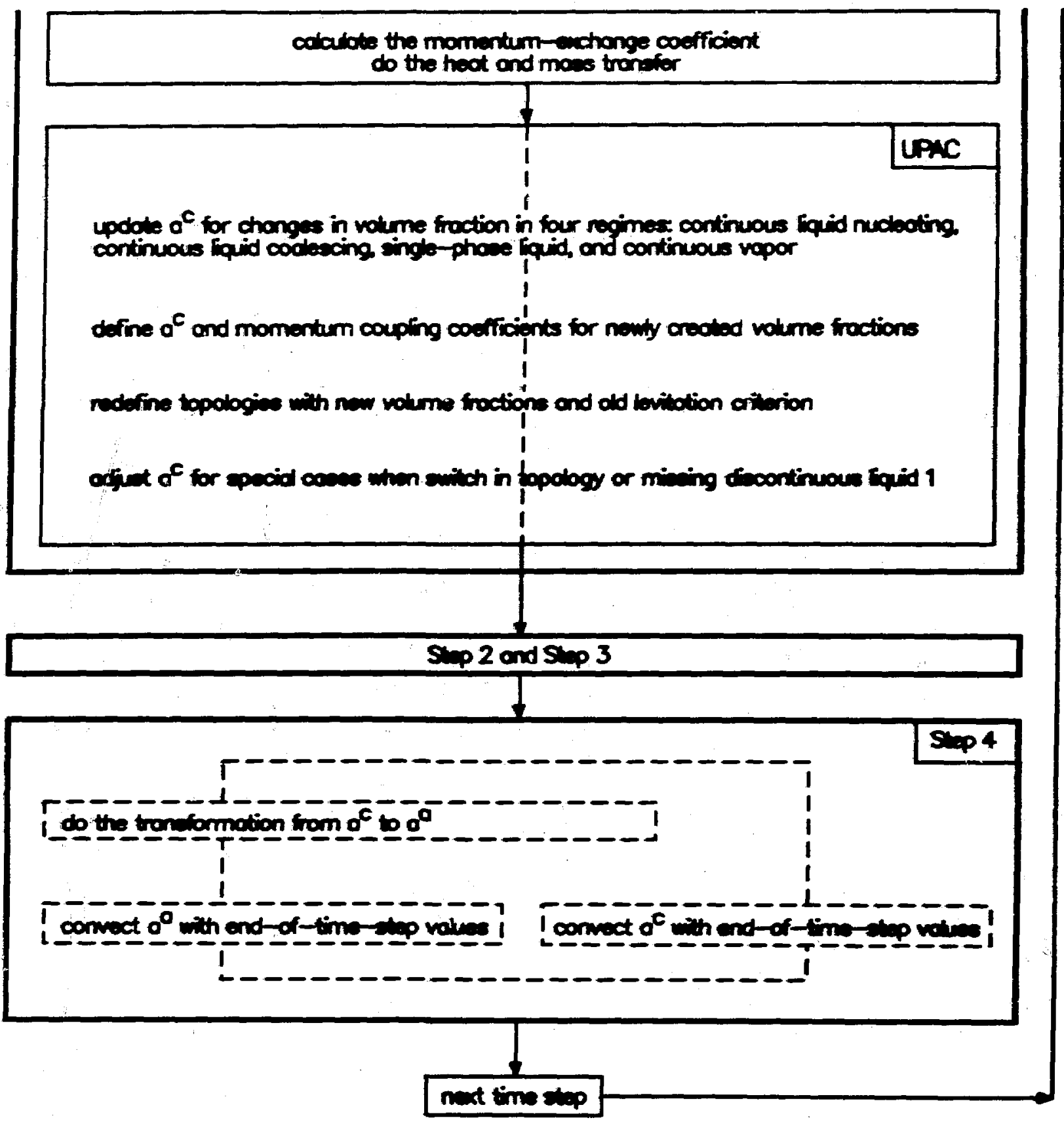

Fig. 1. (cont.) 
describe any contact between two discontinuous velocity fields, between any velocity field and the structure, or between two phases of the same velocity field. Before the start of the transient calculation, the convectible surface areas, $\mathrm{a}^{\mathrm{C}}$, have to be given initial values. This is done in subroutine INICEL (Fig. 1) by using the input values of the radii, $r$, of the equivalent sphere an the known volume fractions, $\alpha$, and

$$
a^{c}=\frac{3 \alpha}{r},
$$

where $\mathrm{a}^{\mathrm{c}}$ is an area concentration or interfacial area per unit volume, given in $\mathrm{m}^{2} / \mathrm{m}^{3}$. There are three values possible for $\mathrm{a}^{\mathrm{c}}$ between the three velocity fields, $a_{L 1 L 2}^{c}, a_{L 1 G}^{c}$, and $a_{L 2 G}^{c}$, wich are the surface areas between the two liquids, between liquid 1 and vapor, and between liquid 2 and vapor, respectively. At least one of the values is zero in a given cell because only the interfacial areas between any velocity field and the continuous velocity field are accounted for. Therefore, the evaluation of $\mathrm{a}^{\mathrm{c}}$ through Eq. (1) is dependent upon the choice of the continuous phase that is done by defining a topology, as described in Sec. 11.

During the transient calculation that involves the boxes Step 1 through Step 4 of Fig. 1, the main tasks of calculational procedures are to provide an update for the convectible surface areas by calculating topologies, source terms, changes in volume fractions, and surface area convection between a given cell and its neighbors. With all convectible surface areas and the cell variables known, the instantaneous interfacial areas are calculated for a given topology.

Mathematically, the procedure to update the convectible surface areas is explicit. This can have a substantial influence on the stability of the calculations. Presently, time-step controls due to these updates have not been introduced because of the lack of experience with the new model approach.

This volume is subdivided so that it follows mainly the computational procedure listed in Fig. 1. First, it describes the topologies and the model for choosing the continuous phase, second, the surface area updates, third, the surface area subdivision into instantaneous interfacial areas, and fourth, the convection of the convectible surface areas. 


\section{Comtimious muse and tomolegr}

Within a computational cell of the Eulerian mesh of AFDM, a maximum of three velocity fields can be present. Twelve topologies are introduced to define the geometrical configuration of fluids and the continuous phase in a given cell. Except for the choice of the continuous phase, the topology selection is made on the presence of velocity fields rather than fluid flow parameters. We use the notation topology because the notation fiow regime includes nore detailed information about the physics of the flow. The subdivision into topologies and groups of topologies helps to set up an efficient logic in the vectorized code.

\section{A. Cont inuous Phase}

The choice whether liquid or vapor forms the cont inuous phase is made by defining the limit at wich the continuous phase changes. This change is allowed to occur within a given range of vapor volume fractions, the lower limit of wich is given by the user-defined constant, acc, and the higher limit by ${ }^{\infty} \mathrm{CD}$. Within this range, a levitation criterion describes the criterion for the transition to vapor continuous flow. Neglecting heat and mass transfer, the momentum exchange with the structure, and convection, the momentum equation for the vapor phase is

$$
\frac{\partial}{\partial t}\left(\bar{\rho}_{G} \vec{v}_{G}\right)=\bar{\rho}_{G} \vec{g}-\alpha_{G} \nabla p+\sum_{m} K_{G, L m}\left(\vec{v}_{L m}-\vec{v}_{G}\right),
$$

where $\bar{p}_{G}$ is the macroscopic vapor density, $\vec{v}_{G}$ and $\vec{v}_{L M}$ are the velocity vectors of the vapor and liquid velocity fields, and $K_{G, L m}$ is the momentum-exchange coefficient, as defined in Sec. III of Vol. III. If droplets are levitated in a vapor flow, and this condition is maintained, the left-hand side (LHS) of Eq. (2) goes to zero. Neglecting the gravitational term against the expectedly large friction term, levitation occurs if the pressure gradient exceeds

$$
\left|\nabla_{p}\right|>p_{T R}^{\prime}=\frac{1}{\alpha_{G}} \sum_{m} k_{G, L m}\left|\vec{v}_{L m}-\vec{v}_{G}\right| .
$$


If the vectors of this equation are not aligned, the angle between the vectors might influence the criterion. The present procedure, however, is only based on absolute values.

The momentum-exchange coefficient is given in Vol. 111 through Eq. (133). If the viscous term is neglected,

$$
K_{G, L m}=\frac{a_{G, L m}}{2} \frac{C_{D}}{4}\left|\vec{v}_{L m}-\vec{v}_{G}\right| \frac{\bar{p}_{G}\left(1-\alpha_{S}\right)}{\alpha_{G}^{2}},
$$

because the dynamic force will dominate the viscous force at the expected velocities. The individual velocity differences $\left|\vec{v}_{L m}-\vec{v}_{G}\right|$ will be associated to the terminal velocities, VDLm, of a single drop of component $m$ in an infinite gas volume

$$
\left|\vec{v}_{L m}-\vec{v}_{G}\right|=v_{D L m} \frac{\alpha_{G}}{1-\alpha_{S}}
$$

This equation, as given in Ref. 1, has been verified for liquid-liquid extractions in spray colums. Using a two-component liquid-vapor system, an expression for voln can be formed. The two steady-state momentum equations are

$$
0=\bar{p}_{G} \vec{g}-\alpha_{G} \nabla p+k_{G, L}\left(\vec{v}_{L}-\vec{v}_{G}\right)
$$

and

$$
0=\bar{p}_{L} \vec{g}-\alpha_{L} \nabla p+K_{G, L}\left(\vec{v}_{G}-\vec{v}_{L}\right)
$$

where $\nabla p$ can be extracted by adding the equation

$$
\nabla_{p}=\frac{\bar{p}_{L}+\bar{p}_{G}}{1-\alpha_{S}}
$$


and substituted into Eq. (6) using Eq (4) together with Eq. (5). With 1 - as $=\alpha_{G}+\alpha_{L}$ and after some manipulations,

$$
G\left(\frac{\bar{\rho}_{L}}{\alpha_{L}}-\frac{\bar{\rho}_{G}}{\alpha_{G}}\right)=\frac{a_{G L} C_{D} \bar{\rho}_{G}}{8 \alpha_{L} \alpha_{G}} v_{D L}^{2}
$$

Using Eq. (1) to replace $30_{L} / a_{G}$ and defining the droplet radius, $r_{d}$, with the Weber number, We $e_{C r i t}=2 r_{d} P_{G} v_{D L /}^{2} \sigma_{L}$, yields

$$
v_{D L}=\left(\frac{4 e_{c r i t} \sigma_{L}\left(p_{L}-\rho_{G}\right) G}{3 C_{D} \rho_{G}^{2}}\right)^{k /} .
$$

Before droplets can break up by reaching the critical Weber number, they are distorted. Fortunately, the terminal velocity of a distorted drop is almost independent of size, as given in Ref. 1 , and is equal to a value calculated by Eq. (10) when using

$$
\frac{{ }_{C r i t}}{C_{D}}=3
$$

This value has been found for fully developed turbulent flow and is expected to be valid at levitation inception when gas or vapor is the continuous phase.

However, Ref. 2 states that the dispersed flow transition proposed by Dukler is consistent with the observed stability of churn-turbulent flom at high velocities. According to Dukler, transition occurs at gas velocities, $v_{G}$, as

$$
\frac{\alpha_{G} v_{G}\left(p_{G}\right)^{\not / z}}{\left[G \sigma_{L}\left(p_{L}-p_{G}\right)\right]^{\not /}}=1.23
$$


if $\alpha_{G}=0.4$. Here, the liquid velocity is snall compared with the gas velocity. Comparing Eq. (12) with Eqs. (10) and (11) by using Eq. (5), the gas velocities differ by a factor of 5.4 with $\alpha_{G}=0.4$.

Reference 3 gives the levitation limit in a tube independent of the tube diameter at

$$
v_{G}=3.1\left[\frac{G \sigma_{L}\left(p_{L}-p_{G}\right)}{\rho_{G}^{2}}\right]^{*}
$$

that is consistent with Eq. (12) at $a_{G}=0.4$. Therefore, it is suggested to use a compromise set of parameters, and take

$$
\frac{m_{\text {crit }}}{C_{D}}=48
$$

in Eq. (10) that would double the terminal velocity. Assuming a constant $C_{D}$ value of 0.45 for fully turbulent flow, the critical Weber number is 22 . The term $C_{D}$ is given that constant value to simplify the choice of user-defined constants, even if the droplet may be distorted and the value of $C_{D}$ higher than 0.45 .

Because Eq. (5) is being confirmed by experiments, the assumptions for deriving this equation sem to be consistent, especially the form of Eq. (4) and hence that of Eq. (136) in Vol. 111, Sec. 111 .

For solid particles, the radius used in defining the Weber numer is given as an input. Now

$$
v_{D L}=\left[\frac{8 r\left(P_{L}-p_{G}\right) G}{3 C_{D} P_{G}}\right]^{1 / 2} .
$$

Substituting Eq. (15) into Eqs. (4) and (3), 


$$
\frac{K_{G, P}}{a_{G}}\left|\vec{v}_{p}-\vec{v}_{G}\right|=\frac{a_{G, P} r\left(\rho_{p}-\rho_{G}\right) \cdot G}{3\left(1-x_{S}\right)} \text {. }
$$

with ag,p $=3$ oxp/r

$$
\frac{K_{G, P}}{\alpha_{G}}\left|\vec{v}_{p}-\vec{v}_{G}\right|=\frac{\alpha_{p}}{1-\alpha_{S}}\left(p_{p}-p_{G}\right) G
$$

that is simply the hydrostatic head of the particles multiplied by their volume fraction. Substituting Eqs. (4), (5), (10), and (17) into Eq. (3),

$$
p_{T H}^{\prime}=\frac{\alpha_{P}\left(\rho_{p}-\rho_{G}\right) G}{1-\alpha_{S}}+\sum_{m=1}^{2} \frac{a_{G, L m}}{4\left(1-\alpha_{S}\right)}\left[\frac{w_{c r i t} C_{D} o_{L m}}{3}\left(p_{L m}-\rho_{G}\right) G\right]^{\frac{1}{2}} .
$$

The value of $p_{T R}^{\prime}$ will be compared to $|\nabla p|$; see Eq. (3). The value of $|\nabla p|$ is calculated in Eq. (22).

Regardless of the levitation criterion, droplet flow will be formed if the vapor-volune fraction is large enough. As the vapor bubbles grow closer, the liquid bridges between them grow thinner. Eventually, the bridges will collapse, and droplets will form for

$$
\frac{\alpha_{G}}{1-\alpha_{S}} \geqq \alpha_{\infty}
$$

where ${ }^{C C D}$ is a user-defined constant. Liquid-continuous flow will always exist for

$$
\frac{\alpha_{G}}{1-\alpha_{S}}<\alpha_{C C},
$$


where acc is a user-defined constant.

The comparison of $p_{T R}^{\prime}$ of Eq. (18) to the absolute pressure gradient is obtained by introducing a relative scale ith an absolute value, $p_{T R}^{+}$, as

$$
p_{T R}^{+}=|\nabla p|-p_{T R}^{\prime},
$$

so that whener $p_{T R}^{+}$is positive, the levitation criterion is met and vaporcontinuous rlow exists. The driving pressure gradient is

$$
\begin{aligned}
|\nabla p|_{i j}= & \frac{1}{2}\left[\left(\frac{p_{i+1, j}-p_{i j}}{\Delta r_{i+z /}}+\frac{p_{i j}-p_{i-1, j}}{\Delta r_{i-z / z}}\right)^{2}\right. \\
& +\left(\frac{p_{i, j+1}-p_{i j}}{\Delta z j+/ z}+\frac{p_{i j}-p_{i, j-1}}{\Delta z j-z / 2}\right)^{2 / / 2} .
\end{aligned}
$$

At the transition between liquid-contimuous and vapor-continuous flow, the calculational procedure for the convectible surface areas and their source terms changes. As described in Sec. III of Vol. 111, the momentum coupling changes substantialty. In consistency with the hysteresis for the momentum coupling coefficients, the choice of the continuous phase is made using a hysteresis on top of Eq. (21). To incorporate the limits of Eqs. (19) and (20), $p_{T R}^{+}$will be forced to be equal to the hysteresis swing of $\Delta p_{T Z}^{\prime}$ by introducing the relative pressure gradient, $p_{T R}^{\star}$, such that

$$
p_{T R}^{*}=p_{T R}^{+} \alpha_{\phi 1}-\Delta p_{T Z}^{\prime}\left(1-\alpha_{\phi 1}\right) \text {, }
$$

where $\alpha_{\phi 1}=\min \left[1,50\left(\frac{\alpha_{G}}{1-\alpha_{S}}-\alpha_{C C}\right)\right]$

both for $\alpha_{C C} \leqq \frac{\alpha_{G}}{1-\alpha_{S}}<0.5\left(\alpha_{C C}+\alpha_{C D}\right)$, 


$$
p_{T R}^{*}=p_{T R}^{+} \alpha_{\phi 2}+\Delta p_{T Z}^{1}\left(1-\alpha_{\phi 2}\right) \text {, }
$$

where $\alpha_{\phi 2}=\min \left[1,50\left(\alpha_{C D}-\frac{\alpha_{G}}{1-\alpha_{S}}\right)\right]$

both for $0.5\left(\alpha_{C C}+\alpha_{C D}\right) \leqq \frac{\alpha_{G}}{1-\alpha_{S}} \leqq \alpha_{C D}$

The hysteresis swing is defined by $\Delta \dot{p}_{\mathrm{TZ}}$, a value given through

$$
\Delta P_{T Z}^{\prime}=\Delta P_{H Y} \cdot G
$$

where $G$ is the invariant gravitational acceleration Isee the discussion following Eq. (44)] and $\Delta$ pHY is a user-defined constant specifying a density difference. If $\Delta$ ptry is set to zero, the hysteresis is turned off. Equations (23) and (24) require that

$$
\alpha_{C D} \geq \alpha_{C C}+0.04
$$

The logic of the hysteresis depends on the values of Eqs. (23) and (24), as well as the previous continuous phase. If liquid is the continuous phase, the phase indicator, 1\%, is zero; if vapor is continuous, the phase indicator is unity. For the present time step, $n+1$, as a function of the previous time step, $n$,

$$
\begin{array}{ll}
i_{\phi}^{n+1}=0 & \text { for } p_{T R}^{*} \leqq-\Delta p_{T Z}^{\prime} \text { or } \\
& \text { for } \Delta p_{T Z}^{\prime} \geq 0 \text { and } i_{\phi}^{n}=0 \text { and }-\Delta p_{T Z}^{\prime}<p_{T R}^{*}<+\Delta p_{T Z}^{\prime} \\
i_{\phi}^{n+1}=1 \quad \text { for } p_{T R}^{*} \geqq+\Delta p_{T Z}^{\prime} \text { or } \\
& \text { for } \Delta p_{T Z}^{\prime}>0 \text { and } i_{\phi}^{n}=1 \text { and }-\Delta p_{T Z}^{\prime}<p_{T R}^{*}<\Delta x_{T Z}^{\prime} .
\end{array}
$$


When solid particulates are more abundant than the liquids, the liquid velocity fields are likely to form the discont inuous phase; see also Sec. 111.B.7. Therefore, the levitation criterion is overridden by

$$
\begin{aligned}
& 1^{n+1}=1 \quad \text { for } \alpha_{L 2}=0 \text { and } \alpha_{p}>C_{P M} \alpha_{L 1} \text { or } \\
& \quad \text { for } \alpha_{L 2}>0 \text { and } \alpha_{P}>\alpha_{L 1}+\alpha_{L 2} \text { and } \frac{\alpha_{G}}{1-\alpha_{S}}>\alpha_{C C},
\end{aligned}
$$

where $C_{P M}$ is a user-defined constant; see Eq. (107).

Figure 2 shows the phase indicator $1 \%$ as a function of the relative pressure gradient, $p_{T R}^{*}$. The solid lines indicate regions where the hysteresis does not apply. The term it is zero for continuous liquid flow, and $1 \phi$ is unity for continuous vapor flow. In the region of the dotted lines, the lower part is taken when moving from left to right with prevailing continuous liquid flow and

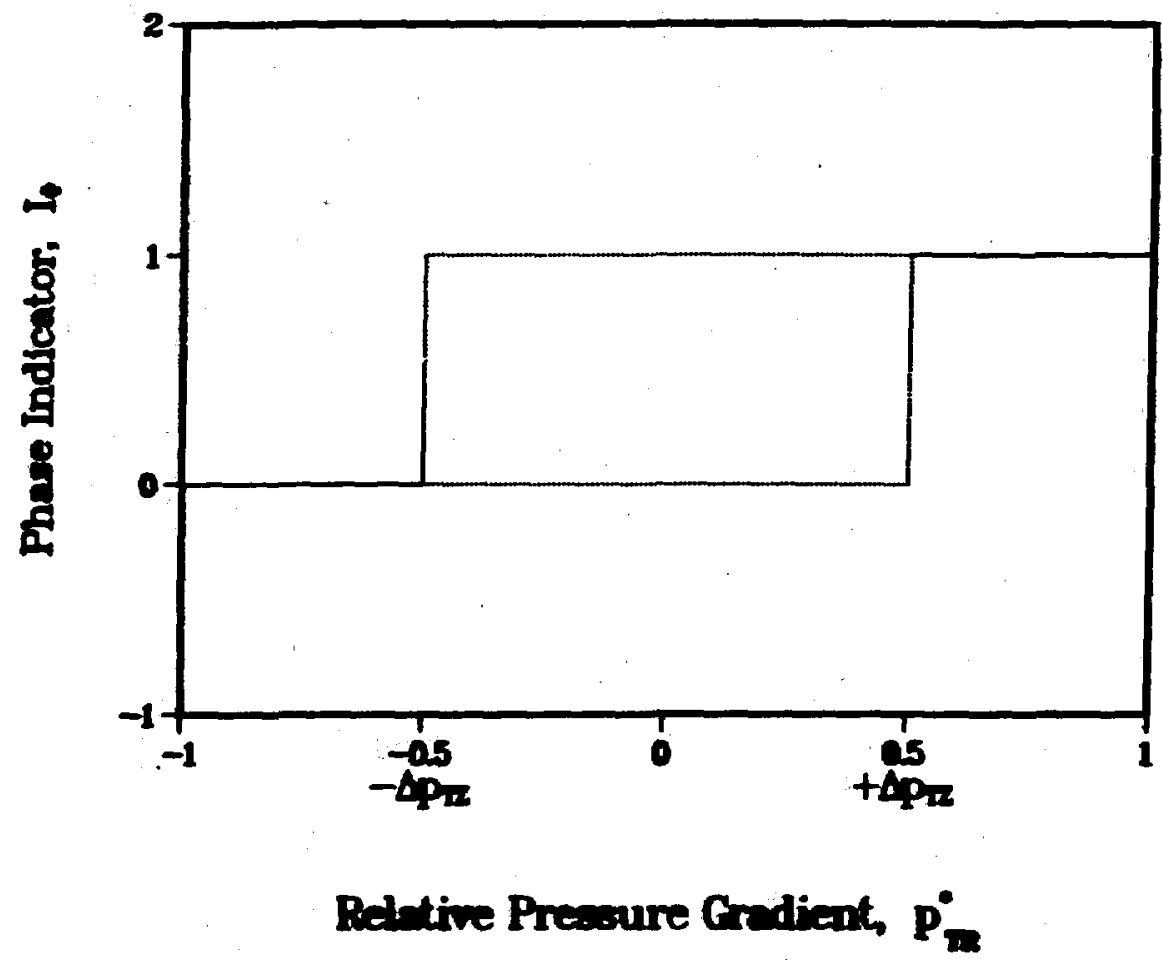

Fig. 2.

Hysteresis outline for the phase indicator. 
increasing $p_{T R}^{*}$ up to the transition at $+\Delta p_{T Z}$. The upper part of the hysteresis is taken when moving from right to left with prevailing continuous vapor flow and decreasing $p_{T R}^{*}$ up to the transition at $-\Delta p_{T Z}^{\prime}$.

\section{B. Cont inuous Liquid}

For all continuous liquid flows with two liquids present in a given cell, the continuous and the discontinuous liquids have to be chosen. No flow pattern information will be used here because the uncertainties connected with available data do not justify a general approach. which is necessary to describe prototypic conditions of reactor materials. Therefore, the choice is simply made on volume fractions of the materials present. The continuous liquid indicator, $\mathrm{I}_{\mathrm{CL}}$, is one if liquid 1 is the cont inuous $\mathrm{liquid}$, and two if liquid 2 is cont inuous:

$$
I_{C L}=1, \quad \text { for } \alpha_{L 2} \leqq\left(1+\alpha_{P F} \alpha_{p}\right) \alpha_{L 1}
$$

and

$$
l_{a}=2, \quad \text { for } \alpha_{12}>\left(1+\alpha_{p F} \alpha_{p}\right) \alpha_{11},
$$

where apf is a user-defined constant that is used as a multiplier of ap to define the influence of the permanently discontinuous solid particulate on the liquid 1-particulate velocity field. The user-defined constant can be used to let liquid 2 become the continuous phase only if the volume fraction of liquid 1 is considerably smaller. At the liquid 1-particulate interface, perfect wetting is assumed so that particulates, if dominant, will be covered by liquid 1. The choice of I $\mathrm{CL}$ will be used in the code to identify the dominant liquid if both liquids are discontinwous.

\section{Topology}

The information about the phase indicator, $1 \$$, and the continuous liquid indicator, I $\mathrm{Cl}$, together with additional volume fractions is used to identify twelve topologies. Additionally, the topology indicator, $I_{T}$, is set to zero 
for no-flow or blocked-flow cells, with anf as a user-defined constant. The topologies are

$$
\begin{aligned}
& I_{T}=0, \text { for } \alpha S \geqq \text { aff } . \\
& I_{T}=1, \text { for } \alpha_{G}>0 \text { and } \alpha_{p}=\alpha_{L 1}=\alpha_{L}=0 \text {, } \\
& I_{T}=2, \text { for } \alpha p+\alpha_{L 1}>0 \text { and } \alpha_{L 2}=\alpha_{G}=0 \text {, } \\
& I_{T}=3, \quad \text { for } \alpha_{L 2}>0 \text { and } \alpha_{p}=\alpha_{L 1}=\alpha_{G}=0, \\
& I_{T}=4, \text { for } \alpha_{p}+\alpha_{L_{1}}>0 \text { and } \alpha_{L 2}>0 \text { and } \alpha_{G}=0 \text { and } I_{C L}=1 \text {, } \\
& I_{T}=5, \text { for } \alpha p+\alpha_{L_{1}}>0 \text { and } \alpha_{L 2}>0 \text { and } \alpha_{G}=0 \text { and } I_{C L}=2 \text {, } \\
& I_{T}=6, \text { for } \alpha p+\alpha_{L 1}>0 \text { and } \alpha_{G}>0 \text { and } \alpha_{L 2}=0 \text { and } I_{\phi}=1 \text {; } \\
& I_{T}=7 \text {, for } \alpha p+\alpha_{L}>0 \text { and } \alpha_{G}>0 \text { and } \alpha_{L 2}=0 \text { and } I_{\phi}=0 \text {, } \\
& I_{T}=8, \quad \text { for } \alpha_{L 2}>0 \text { and } \alpha_{G}>0 \text { and } \alpha_{p}+\alpha_{L 1}=0 \text { and } I_{\uparrow}=1 \text {, } \\
& I_{T}=9, \text { for } \alpha_{L 2}>0 \text { and } \alpha_{G}>0 \text { and } \alpha_{p}+\alpha_{L 1}=0 \text { and } I_{\phi}=0 \text {. } \\
& I_{T}=10, \text { for } \alpha_{p}+\alpha_{L 1}>0 \text { and } \alpha_{L 2}>0 \alpha_{G}>0 \text { and } l_{\phi}=1 \text {, }
\end{aligned}
$$




$$
\begin{aligned}
& I_{T}=11, \text { for } \alpha p+\alpha_{L 1}>0 \text { and } \alpha_{L 2}>0 \text { and } \alpha_{G}>0 \text { and } I_{\phi}=0 \text { and } \\
& I_{C L}=1, \\
& I_{T}=12, \quad \text { for } \alpha p+\alpha_{L 1}>0 \text { and } \alpha_{L 2}>0 \text { and } \alpha_{G}>0 \text { and } I_{\phi}=0 \text { and } \\
& I_{C L}=2 .
\end{aligned}
$$

There are three subsets regarding how many velocity fields are present. For topology 1 through 3 . one velocity field exists, for topology 4 through 9, two velocity fields, and for topology 10 through 12 , three velocity fields. All topologies 6 to 12 represent two-phase flows and the others single-phase flows.

Figure 3 shows the 12 topologies with the discont inuous phases or compo nents represented by spheres. If solid particulates and liquid 1 are discon$t$ inuous, they both share a comon surface and both have an interfacial area connecting them with the continuous phase. The structure volume fraction is added to all topologies indicating the possible contacts, although it has no influence on the selection procedure. For all three-velocity-field topologies, double arrows indicate possible contacts between discontinuous components.

\section{Flow Regimes in Unrestricted Two-Phase Flow}

With the choice of the continuous phase, the dominant flow-regime selec$t$ ion has been made. For a few models, a further subdivision is necessary. However, flow regimes in AFDM are not used to exclusively select models or correlations that have a limited validity. Once the continuous phase is known, most AFDM models apply for the whole range of flow parameters because they are either functions of those parameters or very generalized. In any case, flowregime data in the literature are mostly given for channel flow, like in Ref. 4. The pool geometry of AFD with its unrestricted flow implies that the walls have a negligible influence on most computational cells, and that flow regimes such as annular flow cannot exist. Therefore, we restrict the subdivision to five regimes, the influence of wich on the models is very limited. 
1. Nucleating Regime. For liquid-cont inuous flows at low vapor-volume fractions, the coalescence of bubbles is very limited. If the bubbles are small enough, they can resist coalescence even if they happen to collide. Apparently, surface tension is dominant and the perfectly spherical bubbles act like rigid particles. Therefore, all interfacial source terms are reduced tc nucleation only. According to Ref. 5, small bubbles resist coalescence as long as their radius is smaller than a critical value, rNL, with

$$
{ }_{\mathrm{NL}}=\left(\frac{\mathrm{C}_{\mathrm{NL}}{ }_{\mathrm{CL}}}{\mathrm{Gmax}\left(\Delta \rho_{\min },\left|\rho_{\mathrm{CL}}-\rho_{\mathrm{G}}\right|\right)}\right)^{1 / 2},
$$

where $\sigma_{C L}$ is the surface tension of the continuous liquid against the vapor phase, $C_{N L}$ is a user-defined constant, $\Delta p_{\min }$ is a user-defined minimum density difference, and $G$ is an invariant gravitational acceleration of $9.8 \mathrm{~m} / \mathrm{s}^{2}$. Equation (44), as well as many others in this volume, has been derived from bubble column experiments where the earth's gravity is the body force. In AFDM, the input body force can be set to zero. However, there is no apparent substitute for $\mathrm{G}$ in Eq. (44) and this invariant constant has been introduced to comply with data given in the literature. If $C_{N L}$ is set to 0.1 , the value of the Eortvös number would be 0.4 . With rN known, the limits for the nucleating regime are

$$
\frac{3 \alpha_{G}}{a_{G}}<r_{N L} \text { and } 0<\frac{\alpha_{G}}{1-\alpha_{S}}<\alpha_{N C} \text {, }
$$

where anc is a user-defined volume fraction.

2. Coalescing Regime. For liquid-cont inuous flows with larger vaporvolume fractions and larger bubbles, all interfacial source terms as there are nucleation, turbulence, dynamic forces, and coalescence can be active. Eventually, these source terms are used for the remainder of liquid-continuous flow regimes. As the vapor-volume fraction increases, the number of bubble collisions increases and the influence of one bubble on its neighbors becomes more 


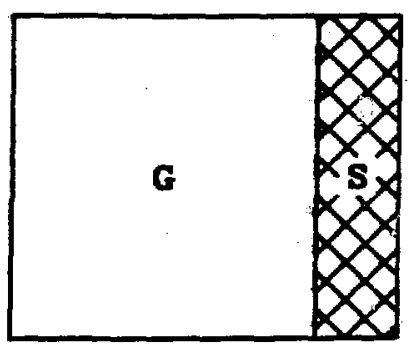

TOPOLOGY 1

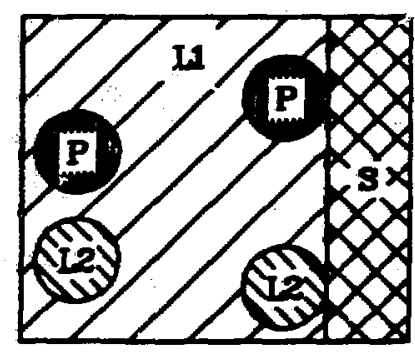

TOPOLOGY 4

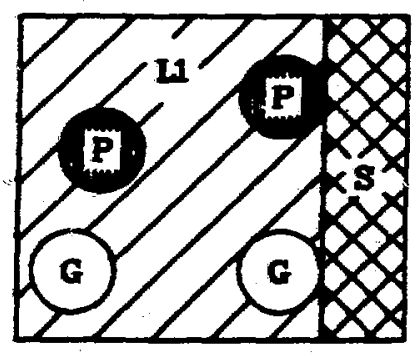

TOPOLOGY ?

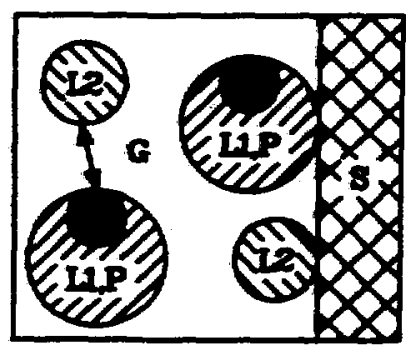

TOPOLOGY 10

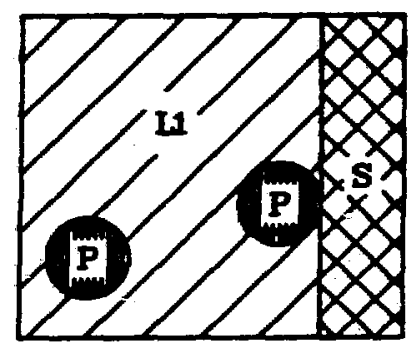

TOPOLOGI 2

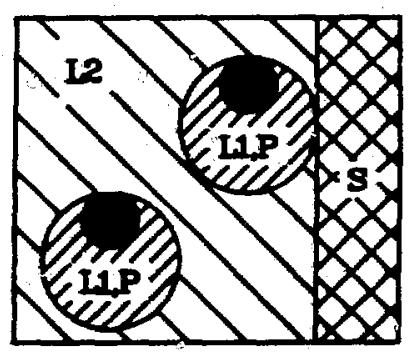

TOPOLOGY 5

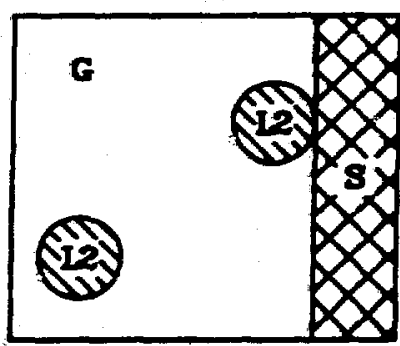

TOPOLOGY $B$

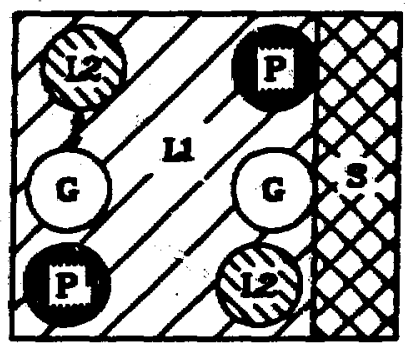

TOPOLOGY 11

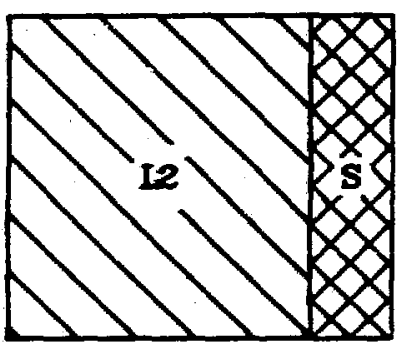

TOPOLOGY 3

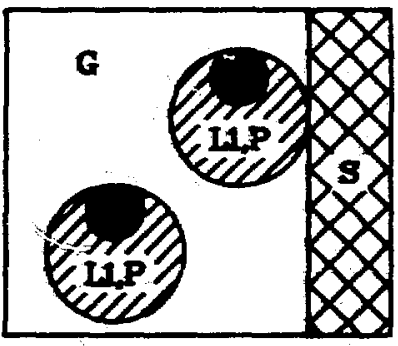

TOPOLOGY 6

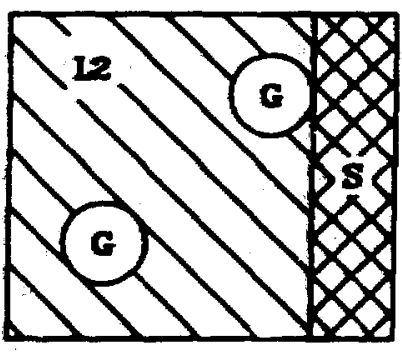

TOPOLOGY 8

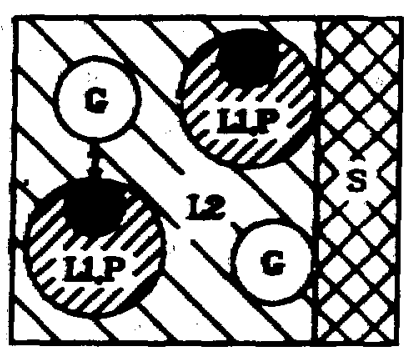

TOPOLOGY 12

I2 liquid 1

G varticles

S structure

Fig. 3.

Topologies represented in AFDM. 
effective. At a vapor-volume fraction of aBFR, the transition to churnturbulent flow is assumed. The threshold value, aBFR, is made a function of the solid particulate volume fraction, with

$$
\alpha_{B F R}=0.3-c_{B C} \frac{\alpha_{P}}{1-\alpha_{S}} \max \left(0.3, \xi_{\sigma}\right),
$$

where $C_{B C}$ is a user-defined constant, and

$$
\begin{array}{ll}
\xi_{\sigma}=\min \left(1, \frac{\sigma_{P}-\sigma_{P L 2}}{\sigma_{L 2}}\right) & \text { for } I_{T}=12 \\
\xi_{\sigma}=1 & \text { for } I_{T} \neq 12 .
\end{array}
$$

where $o_{p}$ is the surface tension of the solid particulates against the vapor phase, oL2 of liquid 2 against vapor, and OPL2 between the particulates and liquid 2. In Ref. 6, the transition to churn-turbulent flaw has been observed to start at a lower void fraction when solid particles are present. In addition, surface tension criteria are supposed to have an effect on the threshold value. The idea is that, for topology 11, perfect wetting is assumed at all times between the continuous liquid 1 and the solid particulates. The lack of solid particulate contact with the other dispersed phases is similar to potential lack of bubble-droplet contact based on surface tensions as discussed in Sec. IV.D. For topology 12, the configuration between solid particulates and bubbles can change with changing surface tensions. As stated in SeC. IV.D., fuel particles and vapor bubbles for prototypic materials are predicted to be in contact below $2822 \mathrm{~K}$ but to largely separate above $2822 \mathrm{~K}$. Therefore, at temperatures close to the $\mathrm{UO}_{2}$ melting point, $\xi_{\sigma}$ is close to unity. As solid particulate-bubble contacts due to surface tensions are increasing with decreasing temperatures, the influence of the particulate on the promotion of churn-turbulent flow is supposed to decrease. Figure 4 shows the values of as a function of the temperature and the particle volume fraction, $x_{p}$, for prototypic materials. The dotted lines represent the volumes for $\xi_{\sigma}=1$ 


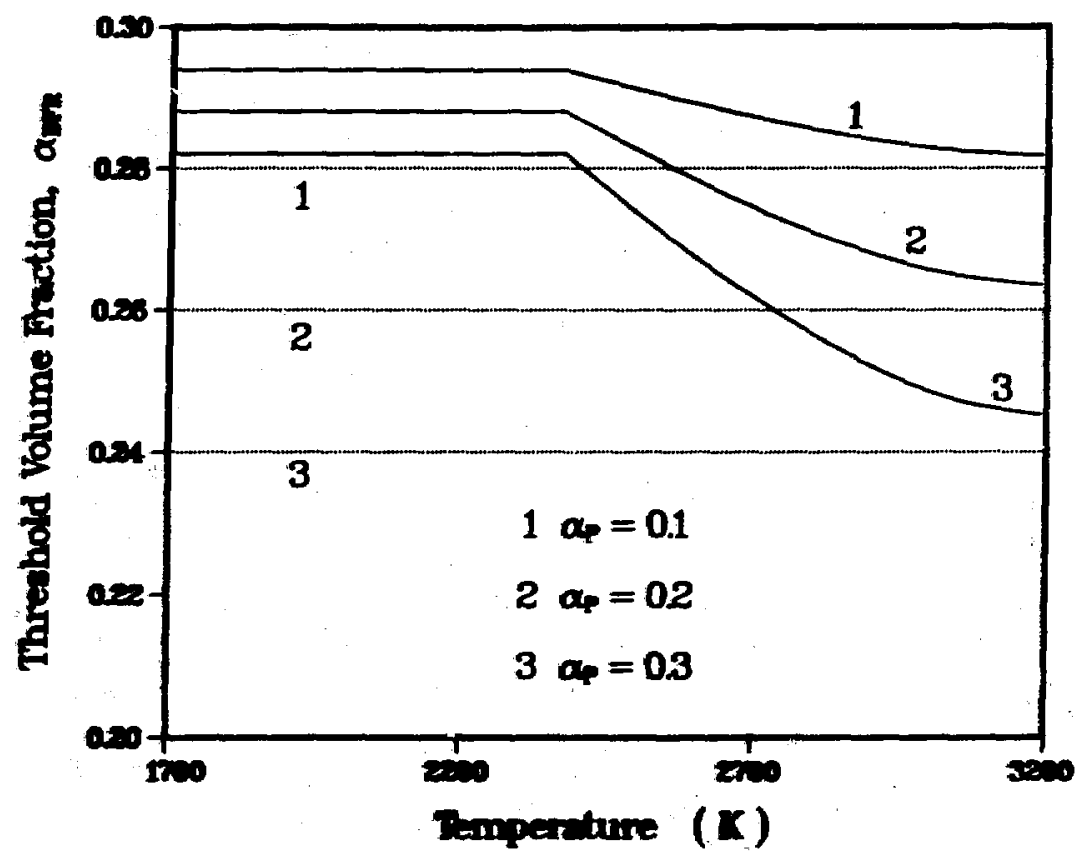

Fig. 4.

The bubbly/churn-turbulent transition threshold.

of topology 11, the solid lines show the variation with temperatures for topology 12. Thermal equilibrium is assumed for all components present in a given cell. Finally, the coalescing regime prevails for

$$
\frac{3 \alpha_{G}}{a_{G}} \geqq r_{M} \quad \text { or } \quad \alpha_{M C} \leqq \frac{\alpha_{G}}{1-\alpha_{S}} \leqq \alpha_{B F R}
$$

3. Churn-Turbulent Regime. For 1 iquid-cont inuous flows and high vaporvolume fractions, the flow pattern becomes highly irregular. Models based on the analysis of single bubbles usually fail.

In Ref. 3, churn flow has been described as an entry region phenomenon wich can only evolve into slug flow after the flow is fully developed. If the velocity is too high, this transition will not take place. However, if the vapor velocity is high enough to lift droplets, droplet flow can be sustained. In the present model, we only deal with unrestricted flow. Therefore, churn flow might extend into cellular flow regime, if vapor velocities are low 
enough. The transition between churn and cellular flow is not very effective in terms of change of physical quantities, such as interfacial area or momentum coupling coefficient. For AFDM, the limit is placed at the user-defined constant, acc. Hence, churn-turbulent regime prevails for

$$
\alpha_{B F R}<\frac{\alpha_{G}}{1-\alpha_{S}} \leqq \alpha_{C C} .
$$

4. Cellular Regine. For liquid-continuous flows with vapor-volume fractions higher than $\alpha_{C C}$, the levitation criterion is turned on and the continuous phase is determined based on the magnitude of the pressure gradients. If the cell pressure gradient is low, levitation does not occur. In the literature, the continuing liquid-continuous flow is described as being churn turbulent rather than cellular. For the AFDM logic, the cellular regine prevails for

$$
\alpha_{C D}>\frac{\alpha_{G}}{1-\alpha_{S}}>\alpha_{C C} \text { and } l_{1}=0
$$

5. Droplet Regime. For vapor-continuous flows, there is only one regime defined. The interfacial source terms for this regime are flashing, dyramic forces, and coalescence. Droplet flow prevails for

$$
\begin{aligned}
& \frac{\alpha_{G}}{1-\alpha_{S}} \geq \alpha_{C D} \text { or } \\
& \alpha_{C D}>\frac{\alpha_{G}}{1-\alpha_{S}}>\alpha_{C C} \text { and } l_{1}=1 .
\end{aligned}
$$




\section{III.}

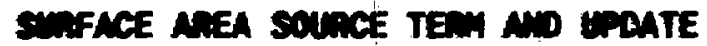

Interfacial surface areas in AFDM are functions of time and space. We therefore call them convectible surface areas. Although they cannot be conserved, a convection equation can be written with the general form

$$
\frac{\partial a_{f}^{c}}{\partial t}+\nabla \cdot\left(a_{f}^{c} \vec{v}_{q}\right)=\sum_{A} S_{A, f}+\sum_{B} S_{B, f}+s_{C, f}
$$

where $a^{c}$ is the convectible surface area per unit volume with the subscript, $f$, standing for $L 1 L 2, G L_{1}$, and $G_{2}, \vec{v}_{q}$ is the velocity of momentum field $q$ to be used to convect $a_{f}^{C}, S_{A}, f$ is the source term for contincous liquid flow, $S_{B, f}$ for continuous vapor flow, and $S_{C}, f$ for change in volume fraction due to mass transfer within a given cell. Equation (52) is solved in three steps. First, the second term of the LHS is set to zero and the equation is solved with all source terms $S_{A, f}$ and $S_{B}, f$, wich are defined in subrout ine INIIFA. Second, after having done the heat- and mass-transfer operations, the same equation is solved with $S_{C}, f$ knom from the volume fraction updates. The source terms for these two steps are described in this section. Third, the source terms are se: to zero and the surface areas are convected with end-of-time-step velocities. This part is described in Sec. $V$.

The right-hand side (RHS) of Eq. (52) is written as the sum of independent source terms. By this we anticipate that the physical processes leading to the source terms are independent of each otrer. By introducing a radius, $r$, of the equivalent sphere in Eq. (1), the radius, the volume fraction, and the convectible surface area are interdependent and all processes have to be described with the reference to a representative sfoterical discont inuous fluid particle. Not only has the radius, $r$, to represent an average over all fluid particles present in a given cell but also it has to take-into account the change in surface area if the fluid particles are nonspherical. ust source term models presented here refer to an equilibrium radius that is likely to exist under the present cell conditions, and a time constant to reach this equilibrium. This method is consistent with the use of independent source terms since it allows superposition of different physical processes. Consequently, any new source 
term wodel identified as being independent for a given flow problem can simply be added. For liquid-continuous flow, two source terms are assumed not to be independent of each other. These are dynamic forces for bubbles and buoyancydriven turbulence breakup. A common equilibrium radius and a comen time constant have to be provided for both processes.

The convectible surface area $a_{i}^{c}$ standing for $a_{L 1 L 2}^{c}, a_{L 1 G}^{c}$, and $a_{L 2 G}^{c}$ is defined for the interfaces between the discontinuous components and the cont inuous phase only. If liquid 1 is discont inuous, the solid particulates do not contribute to the convectible surface area. If a component is missing in a cell, the convectible surface area of its contact to the cont inuous phase is zero. Table I lists the convectible surface areas with non-zero values for the twelve topologies.

\section{A. Update Due to Hydrodynamic Models}

The first step of updating the convectible surface areas involves the calculation of the source terms $S_{A}, f$ and $S_{B}, f$ for continuous liquid and cont inuous vapor flows, respectively, and the explicit solution of $\mathrm{Eq}$. (52) with the convection term and $\mathrm{S}_{\mathrm{C}}, \mathrm{f}$ set to zero.

$$
\frac{d a_{f}^{c}}{d t}=\sum_{A} s_{A, f}+\sum_{B} s_{B, f}
$$

There are three different sets of regimes, for wich the of Eq. (53) stays the same. Wefer to the flow regimes of Sec. H.D. First, for the nucleating regime, all $S_{B}, f$ are zero, and only one source term is active for $S_{A}, f$. Second, for coalesing, churn-turbulent, and cellular regimes, all $S_{B}, f$ are zero, and $S_{A}, f$ include nucleation, splitting, and coalescence. Third, for the droplet regime, all $S_{A}, f$ are zero, and $S_{B}, f$ include splitting and coalescence. Two models for splitting process are presently used.

To specify $S_{A}, f$ and $S_{B}, f$, physical models have to provide the constitutive relationships. The first model includes the calculation of an equilibriun surface area based on the cell variables and a time constant with which the equilibrium is approached. In the second model, the equilibrium surface area 


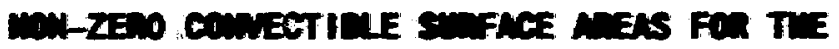

12 AFIn TOMLLSIES

\begin{tabular}{|c|c|c|c|c|c|}
\hline Topology & $\begin{array}{l}\text { Cont inuous } \\
\text { Momentum } \\
\text { Fields } \\
\end{array}$ & $\begin{array}{c}\text { Discont inuous } \\
\text { Momentuin } \\
\text { Fields } \\
\end{array}$ & $\begin{array}{c}\text { Solid } \\
\text { Particulate } \\
\text { Possible } \\
\end{array}$ & $\begin{array}{c}\text { Convect ible } \\
\text { Sur face } \\
\text { Areas } \\
\end{array}$ & $\begin{array}{c}\text { Film } \\
\text { Boiling } \\
\text { Possible }\end{array}$ \\
\hline 1 & G & & & & \\
\hline 2 & L1 & & $x$ & & \\
\hline 3 & L2 & & & & \\
\hline 4 & L1 & 12 & $x$ & $a_{L 1 L 2}^{c}$ & \\
\hline 5 & $\mathrm{~L} 2$ & L1 & $x$ & $a_{11 L 2}^{c}$ & \\
\hline 6 & $\mathbf{G}$ & L1 & $x$ & $a_{G}^{c}$ & \\
\hline 7 & L1 & $G$ & $x$ & $a_{Q 1}^{c}$ & \\
\hline 8 & G & L2 & & $a_{G 2}^{c}$ & \\
\hline 9 & $\mathrm{~L} 2$, & $G$ & & $a_{G 2}^{c}$ & \\
\hline 10 & G & $L 1, L 2$ & $x$ & $a_{G 1,}^{c}, a_{G 2}^{c}$ & \\
\hline 11 & L1 & $12, G$ & $x$ & $a_{11,12}^{c}, a_{G 1}^{c}$ & $x$ \\
\hline 12 & 12 & $L 1, G$ & $x$ & $a_{L 1, L 2}^{c}, a_{G, 2}^{c}$ & $x$ \\
\hline
\end{tabular}

is not available, but a coalescence rate is available. Writing the futs of Eq. (53) in the form of the two model contributions yields

$$
\frac{d a_{f}}{d t}=\sum_{k} \frac{a, k^{-a_{f}}}{\tau_{k}}-a_{f}^{2} r_{c} .
$$

where $a$ is a simplified form of writing $\mathrm{a}^{\mathrm{C}}$, a $\mathrm{a}_{e}$ is the equilibrium surface area, $\tau$ is the time constant, and $\Gamma_{C}$ is the coalescence rate. The number of models for the first term of the PAS is $k=1$ for the nucleating regine where $\mathrm{r}_{\mathrm{c}}=0, k=2$ for coalescing, churn-turbulent, and cellular regime, and $k=2$ for the droplet regime. Using the approximation $\left(a_{f}\right)^{2}=a_{f}^{n+1} a_{f}^{n}$ in Eq. (54) and solving for a given time increment, $\Delta t$, would yield 


$$
a_{f}^{n+1}=\frac{\sum_{k} a_{e, k} \frac{\Delta t}{\tau_{k}}+a_{f}^{n}}{1+\Delta t\left(\sum_{k} \frac{1}{\tau_{k}}+a_{f}^{n} r_{c}\right)},
$$

where the superscript $n$ and $n+1$ denote the last and present time steps, respectively. The explicit solution yields

$$
a_{f}^{n+1}=a_{f}^{n}\left[1-\left(\sum_{k} \frac{1}{\tau_{k}}+a_{f}^{n} r_{c}\right) \Delta t\right]+\sum \frac{\Delta t}{\tau_{k}} a_{e, k} .
$$

The explicit solution will be used. However, to prevent over shooting, the time constants and $r_{C}$ have to be limited. For small $\tau$ and large $I_{C}$, the implicit solution yields

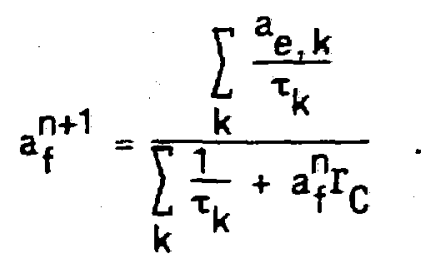

Combining Eqs. (57) and (56) and solving for $\Delta t$.

$$
\Delta t=\frac{1}{\sum_{k} \frac{1}{\tau_{k}}+a_{f}^{n} \Gamma_{C}} .
$$

This is the maximum time-step size that can be used with the explicit solution. Eq. (56), without overshooting the equilibrium interfacial area. For large $t$ ine steps when

$$
\Delta t\left[\left[\frac{1}{\tau_{k}}+a_{f}^{n} r_{c}\right]>T\right.
$$


to use Eq. (56), $\tau_{k}$ should be increased and/or $I_{c}$ decreased so that Eq. (58) is satisfied. Consequently, the limits are given by

$$
\frac{1}{\tau_{k}}=\min \left(\frac{1}{\tau_{k}}, \frac{1}{\left(\sum_{i} \frac{1}{\tau_{i}}+a_{f}^{n} \Gamma_{C}\right) \tau_{k} \Delta t}\right)
$$

and

$$
I_{C}=\min \left(I_{C} \cdot \frac{r_{C}}{\left(\sum_{i} \frac{1}{\tau_{i}}+a_{f}^{n} r_{C}\right) \Delta t}\right) .
$$

If the limits of Eqs. (60) and (61) are reached, the updated area will be equivalent to the equilibrium area for the given cell variables. If more than one source term model affects the convectible surface area, the equilibrium is a function of the individual equilibrium areas, the time constants, and the coalescence rate. Usually, the time constants are indirectly proportional to the convectible surface areas so that the product af $\tau$ is constant. Therefore, the equilibrium convectible surface area for a given cell can be formed by setting the time derivative of Eq. (54) to zero. For given constant rates $r_{\tau}=1 /$ (afr), the equilibrium surface area, $a_{e}, f$, yields

$$
a_{e, f}=\frac{\sum_{k, k} r_{e, k}}{\sum_{k} r_{\tau, k}+r_{C}} .
$$

Equation (62) can be used to easily assess the magnitude of the equilibrium value. However, the relevant update for the code is given through Eqs. (56), $(60)$, and (61).

The update of Eq. (56) needs to restrict splitting processes to only increase the value of $a^{n+1}$, and coalescence to only decrease it. Additionally, the convectible surface area is limited by user-defined radii. Equation (56) for droplets yields 


$$
\begin{aligned}
a_{f}^{n+1} & =\max \left(\frac{3 \alpha}{r_{x d}}, \min \left(\frac{3 a}{r_{m d}}, a_{f}^{n}\right.\right. \\
& \left.\left.+\left[\sum_{k} \frac{1}{\tau_{k}} \max \left(0, a_{e, k}-a_{f}^{n}\right)-a_{f}^{n} a_{f}^{n} r_{c}\right] \Delta t\right)\right)
\end{aligned}
$$

where $\alpha$ is the volume fraction of the droplets, and $r_{x d}$ and $r_{\text {md }}$ are the userdefined aximum and minimum droplet radii, respectively. Equation (56) for bubbles yields

$$
\begin{aligned}
a_{f}^{n+1} & =\max \left(\frac{3 \alpha}{r_{x b}}, \min \left(\frac{3 \alpha}{r_{m b}}, a_{f}^{n}\right.\right. \\
& \left.\left.+\left[\sum_{k} \frac{1}{\tau_{k}} \max \left(0, a_{e, k}-a_{f}^{n}\right)-a_{f}^{n} a_{f}^{n} r_{c}\right] \Delta t\right)\right),
\end{aligned}
$$

where $\alpha$ is the volume fraction of bubbles, and $r_{x b}$ and $r_{\text {nd }}$ are the user-defined maxim and minimm bubble radii, respectively. The limit rxb limits the convectible surface area at small values. As described in Sec. III.B.1, the nucleation model also limits the area through the minimm number density, $n_{\text {min }}$, and Eq. (65). Since the time constant of the nucleation is usually snall, this limit might dominate over the limit given by $r_{x b}$.

\section{B. Surface Area Source Terms}

The source terms for the update of the convectible surface areas are defined by the equilibrium surface areas and the time constants, or by the coalescence rate; see Eq. (54). The equilibrium surface areas are usually functions of equivalent radii with Eq. (1) specifying the functional relationstip. However, for the nucleation nodel, the number of bubbles per unit cell volume, $n_{b}$, is calculated so that

$$
a^{c}=\left(36 \pi n_{b}\right)^{x_{3}} \alpha^{x}
$$

Note that $n_{b}$ is not given as per fluid volume. 
The following sections describe physical models for the source terms. The surface tensions mentioned therein are calculated using interface temperatures as defined in Sec. IV.H.

1. Bubble Nucleation. The bubble nucleation model is used in all liquidcont inuous regimes to describe the increase in convectible surface areas as a function of the superheat, $\Delta T_{S u p}$, of the liquid phase. Nucleation is assumed to occur only in the bulk liquid so that

$$
\Delta T_{\text {Sup ,m }}=T_{\text {Lm }}-T_{\text {Sat, },} \text {, }
$$

where $T_{L m}$ is the bulk liquid temperature of energy component $m$, and $T_{\text {Sat, }} m$ is its saturation temperature corresponding to the partial pressure of this material in the vapor field. Reference 7 provides data about the opt imum bubble number density as a function of the liquid superheat used to recalculate nonequilibrium flashing of water in a converging-diverging nozzle. For the use with other materials, a dimensionless liquid superheat, $\theta_{\text {sup, }}$ is used with

$$
\theta_{\text {Sup }}=\max \left[0, \frac{\Delta T_{\text {Sup }}}{T_{\text {crit }}} \min \left(30, \frac{p_{\text {crit }}}{p_{\text {crit }}-p_{\text {Sat }}}\right)\right] \text {, }
$$

where $P_{c r i t}$ and $T_{c r i t}$ are the critical pressure and temperature, respectively. The data of Ref. 7 are approximated by an exponential function with userdefined limits. The equilibrium bubble number density, $n_{b}$, is

$$
\begin{gathered}
n_{b}=n_{\text {min }}\left(1-\alpha_{S}\right) \text { for } \theta_{\text {Sup }} \leqq \theta_{0} \text { and } p_{\text {Sat }} \leqq 0.9 p_{\text {crit }} . \\
n_{b}=\left(n_{\text {min }}+n_{\max }\left[1-\exp \left(-C_{\theta}\left(\theta_{\text {Sup }}-\theta_{0}\right)^{2}\right)\right]\right)\left(1-\alpha_{S}\right) \\
\text { for } \theta_{\text {Sup }}>\theta_{0} \text { and } p_{\text {Sat }} \leqq 0.9 p_{\text {crit }} \\
n_{b}=\left(n_{\min }+n_{\max }\right)\left(1-\alpha_{S}\right) \quad \text { for } p_{\text {Sat }}>0.9 p_{\text {crit }},
\end{gathered}
$$


where $n_{\min }$ and $n_{\max }$ are the minimum and maximum number densities per fluid volune, respectively, and $\theta_{0}$ and $C_{\theta}$ are additional user-defined constants. Figure 5 shows the bubble number density of $E q$. (68) as a function of the dimensionless liquid superheat, $\theta_{\text {sup. The }}$ circles show the opt imum bubble number density at nucleation inception of Ref. 7 used to recalculate water flashing in a converging-diverging nozzle. However, the data base of these experiments is limited, and the number density was used as initial condition for the two-phase section, a reason why the number density decreases for higher values of superheat. Here, we do not follow this approach but use the ascending branch of the data to evaluate the increasing potential of generating bubbles with increasing superheat.

In topologies 11 and 12 , a second liquid is present in a continuous liquid cell with bubbles. Here, the number density is calculated for both liquids, $n_{b, L 1}$ for liquid 1 , and $n_{b}, L 2$ for liquid 2 , and then is combined with

$$
n_{b}=n_{b, L 1}+n_{b, L 2}
$$

The bubble nucleation model does not apply for single-phase liquid cells. The time constant to achieve equilibrium is based on a constant volume cuuc which is a user-defined constant. To incorporate the effect of higher vapor-volume fractions that can inhibit nucleation by the presence of already existing bubbles, the effective time constant is given by

$$
\tau=\tau_{M U C} \exp \left[-\frac{\frac{\alpha_{G}}{1-\alpha_{S}}-\alpha_{N C}}{C_{N C}\left(\alpha_{C C}-\alpha_{N C}\right)} \ln \tau_{M U C}\right],
$$

where $C_{A C}$ is a user defined constant, and acc and anc are user-defined constants as show in Sec. 11.D. If $\mathrm{CAN}_{\mathrm{NC}}$ is zero, the bubble nucleation is used only in the nucleating regime. Figure 6 shows the time constant, $\tau$ wuc, as a function of the vapor-volume fraction using the default user-defined constants.

For the nucleating regime, bubble nucleation is the only surface area source term. The limiter of Eq. (60) reduces therefore to 


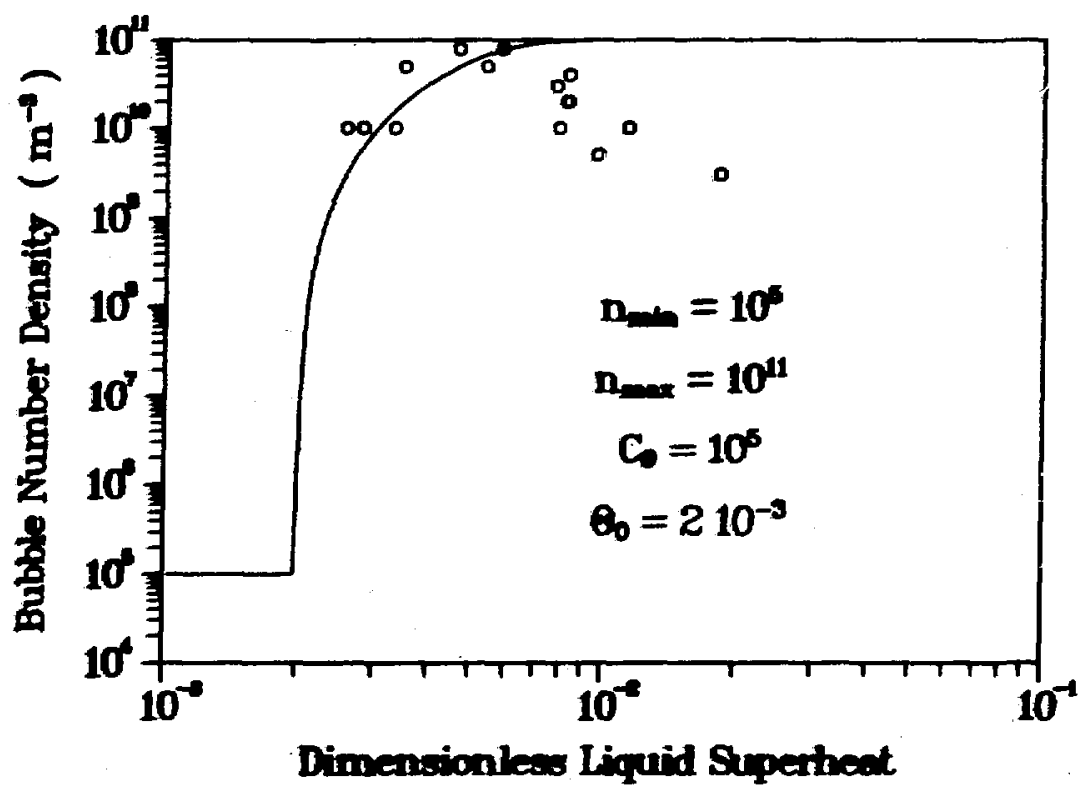

Fig. 5 .

Equilibrium bubble number densities used to calculate water flashing in a converging-diverging nozzle.

$$
\frac{1}{\tau}=\min \left(\frac{1}{\tau}, \frac{1}{\Delta t}\right)
$$

and the updated surface area is equal to the equilibrium area for all time constants smaller than $\Delta t$. The bubble nucleation model is coded in subroutine MuCL.

2. Dynamic forces for Bubbles. The dynamic fcrce model for bubbles is used in the coalescing, churn-turbulent, and cellular regimes. It is based on a Weber criterion with the velocity between vapor and the continuous liquid as main parameter. The velocity difference, $\Delta v$, is

$$
\Delta v=\max \left(10^{-4},\left|\vec{v}_{G}-\vec{v}_{C L}\right|\right)
$$

where $\vec{v}_{G}$ and $\vec{v}_{C L}$ are the velocities of the vapor and the continuous liquid, respectively. The Weber criterion is extended using Ref. 8 . In case that 


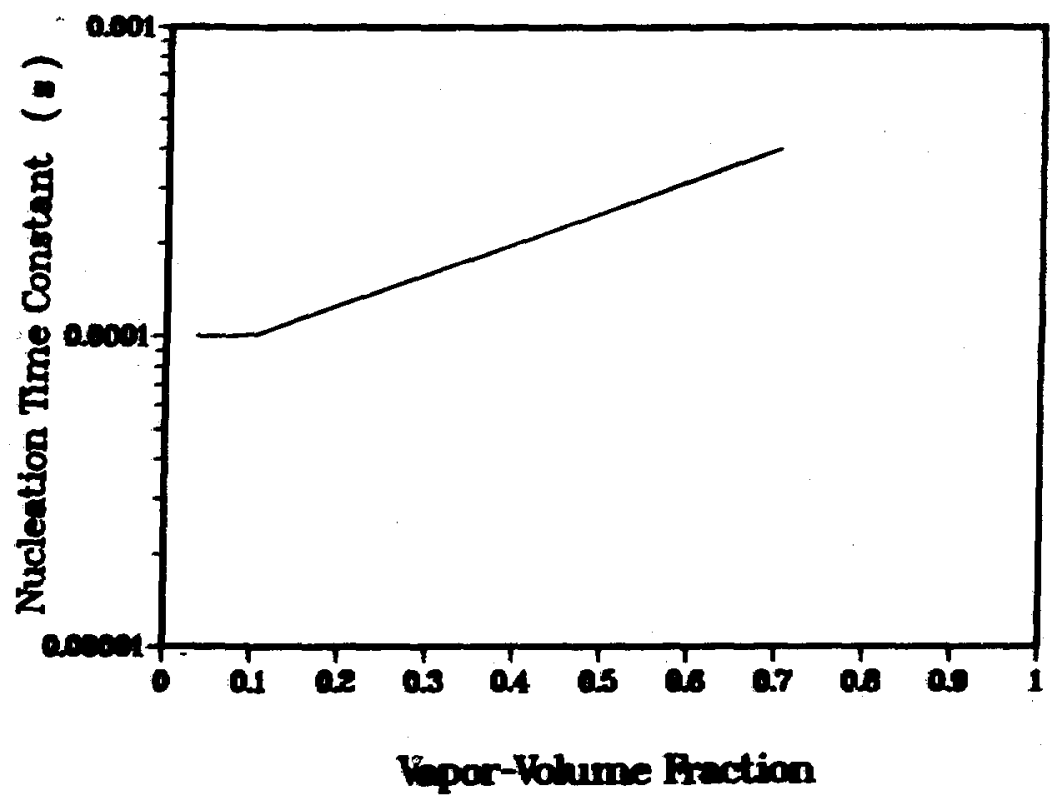

Fig. 6.

Mucleation time constants using the AFDM default model parameters.

equilibrium is achieved between the forces acting on the bubble surface, the equilibrium radius, $r_{e}$, is given by

$$
e_{\mathrm{FB}}+\frac{7 \mu_{\mathrm{b}}^{2}}{\rho_{\mathrm{b}} r_{e}^{\sigma}}=\frac{\rho_{\mathrm{CL}}{ }^{2 r_{e}} e^{\Delta v^{2}}}{\sigma}
$$

where Wefb is a user-defined bubble Weber number, $\sigma$ is the surface tension between the continuous liquid and vapor, $\mu$ is the dynamic viscosity, and $p$ the microscopic density. Solving for the re yields

$$
r_{e}=\frac{{ }_{F b}{ }_{F}}{4 \rho_{C L} \Delta v^{2}}+\left[\left(\frac{{ }_{e} e_{F B}{ }^{o}}{4 \rho_{C L} \Delta v^{2}}\right)^{2}+\frac{7}{2} C_{F V} \frac{\mu_{b}^{2}}{\rho_{b} \rho_{C L} \Delta v^{2}}\right]^{0.5},
$$

where $C_{F V}$ is a user-defined constant. 
The equilibrium surface area, ae, is now

$$
a_{e}=\frac{3 \alpha_{G}}{r_{e}} .
$$

For the time constant, $\tau$, we use data from Ref. 9 where the time interval to reach equilibrium, $\Delta t_{e}$, is given by

$$
\Delta t_{e}=5.6 \frac{r_{b}}{\Delta v}\left(\frac{P_{b}}{\rho_{C L}}\right)^{1 / 2} .
$$

The formula was slightly changed to reduce the breakup at high velocity differences. Now, the time constant is

$$
\tau=2 C_{F T} \frac{r_{b}}{\Delta V} \max \left(1, \frac{\Delta V}{\Delta V_{R T}}\right)\left(\frac{P_{b}}{P_{C L}}\right)^{1 / 2},
$$

where $C_{F T}$ is a user-defined constant, and $\Delta V_{R T}$ is a user-defined velocity difference. The dynamic force model for bubbles is coded in subroutine DMFOR. Figure 7 shows an example for the equilibrium radius and time constant during breakup of bubbles by dynamic forces. The parameters are chosen to be similar to those in a molten $\mathrm{LO}_{2}$-pool, with $\sigma=0.5 \mathrm{~N} / \mathrm{m}, P b=2 \mathrm{~kg} / \mathrm{m}^{3}, \mathrm{PCL}=8000$ $\mathrm{kg} / \mathrm{m}^{3}$, and $\mathrm{mb}=6 \cdot 10^{-7} \mathrm{~m}^{2} / \mathrm{s}$. The default values of the user-defined constants and weFB $=10$ are used. The time constant is calculated with $r_{b}=r_{e}$.

3. Dynamic Forces for Droplets. The dynamic force model for droplets is used for droplets in all regimes. It is similar to the dynamic force model for bubbles. We refer to Sec. III.B.2. The velocity difference is defined by

$$
\Delta v=\max \left(10^{-4},\left|\vec{v}_{D L}-\vec{v}_{C P}\right|\right),
$$


where the subscripts $\mathrm{DL}$ and $\mathrm{CP}$ denote the discontinuous liquid and the cont inuous phase, respectively. The maximum stable size of the droplets is estimated through a modified Weber criterion. The estimates for the size is taken from Ref. 10 where the ratio of the densities has been identified as a parameter

$$
c_{p}=\left(\frac{\rho C P}{\rho_{d}}\right)^{3 / 2}
$$

where the subscript $d$ represents the discontinuous droplet. The correlation of Ref. 10 for liquid-liquid systems is used because its values approach those of the gas-liquid correlation for $C_{\rho} \leqq 0.002$ so that it is a reasonable approximation in all regimes. We follow a very simple approximation by setting the dimensionless breakup time and the drag coefficient at fragmentation constant. The approximation yields a maximum stable droplet radius, $r_{\max }$, with

$$
r_{\text {max }}=\frac{{ }^{e_{F D}}{ }}{2 \rho_{C P} \Delta v^{2}}\left(1-\frac{10 C_{\rho}}{1+10 C_{\rho}}\right)^{-2},
$$

where $\sigma$ is the surface tension between the droplet and the cont inuous phase, and WeFD is a user-defined droplet Weber number. It should be noted that this model is not the only one to describe the fragmentation of droplets. However, unlike for bubbles, a turbulence breakup model is not avaifable, and the correlation above would yield large sizes for small velocity differences and small density ratios. Therefore, introduce a size limit based on a limiting Eơt vös number. Eventually, the equilibrium radius is

$$
r_{e}=C_{F D} \min \left(r_{\max },\left[\frac{\sigma}{G \max \left(0.1, \operatorname{abs}\left(P_{C P}-P_{d}\right)\right)}\right]^{0.5}\right),
$$

where $C_{F D}$ is a user-defined constant. Again, taking a constant dimensionless time for the completion of the breakup process given in Ref. 10, a simple approximation for the time constant, $\tau$, of the fragmentation becomes available as 


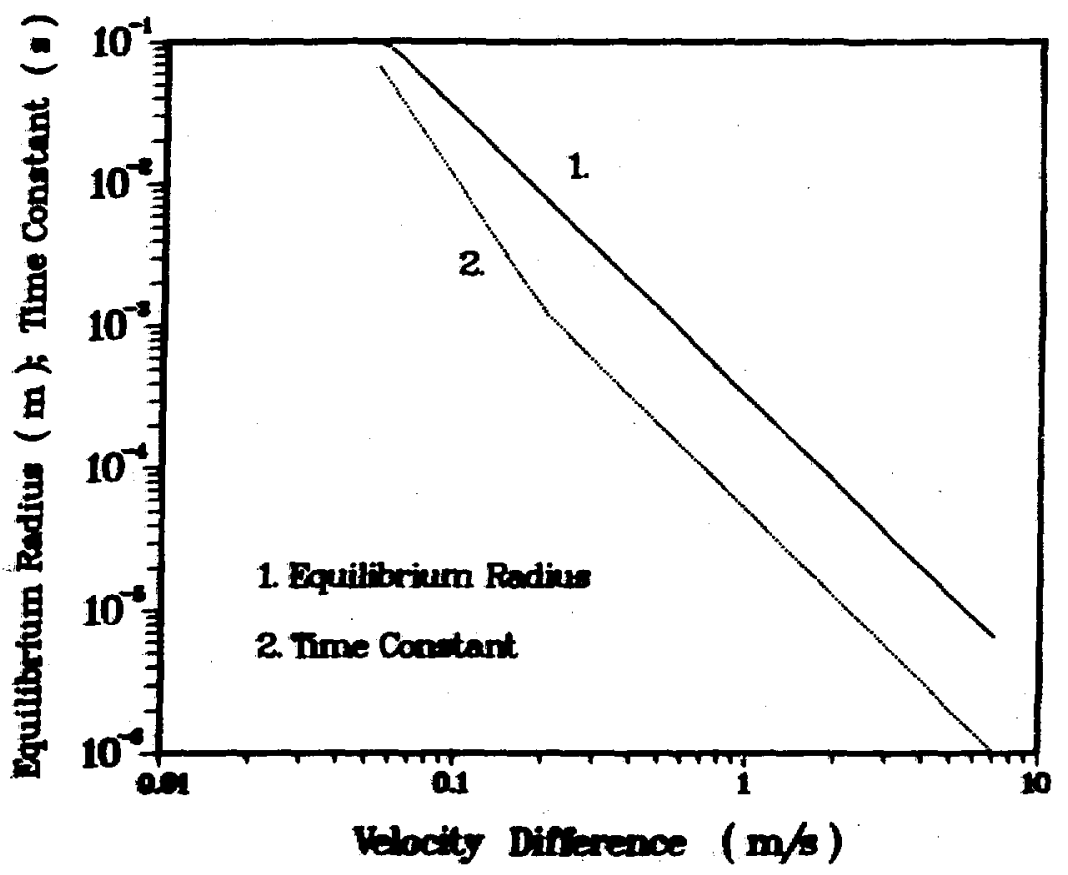

Fig. 7.

Example of the breakup of bubbles by dynamic forces.

$$
\tau=C_{F S} \frac{r_{d}}{3 \Delta v C_{p}}
$$

where $C_{F S}$ is a user-defined constant, and $r_{d}$ is the droplet radius of the previous time step. The equilibrium surface area, $a_{e}$, is

$$
a_{e}=\frac{3 \alpha_{D}}{r_{e}}
$$

The dynamic force model for droplets is coded in subrout ine DYnDro.

Figure 8 shows an example for the equilibrium radius and time constant during breakup of droplets by dynamic forces. The parameters are chosen to be similar to those in a molten $U_{2}-P 001$, with $P C P=10 \mathrm{~kg} / \mathrm{m}^{3}, P d=8000 \mathrm{~kg} / \mathrm{m}^{3}$, and $\sigma=0.5 \mathrm{~N} / \mathrm{m}$. The default values of the user-defined constants and 
WeFD $=13$ are used. The time constant is calculated with $r_{d}=r_{e}$. For velocities below $20 \mathrm{~m} / \mathrm{s}$, the second term of Eq. (81) limits the equilibrium radius to about $1 \mathrm{~mm}$.

4. Buoyancy-Driven Turbulence Breakup. The turbulence breakup model for bubbles is used in the coalescing, churn-turbulent, and cellular regimes. As described in Ref. 11, a bubble can break up if its eigenfrequency corresponds to the frequency of the forces originating from turbulent eddies. As the turbulence energy of the continuous phase in vapor-continuous flows is smaller than in liquid-continuous flows, this model is not used in the droplet regime. The characterizing frequency, $f$, of the turbulent flow is

$$
f=\frac{1}{2 r}\left(\bar{v}^{2}\right)^{1 / 2}
$$

where $\bar{v}^{2}$ is the spatial average of the square of the velocity differences over a distance $2 r$. The natural frequency, $f_{b}$, of a spherical bubble is

$$
\left(2 \pi f_{b}\right)^{2}=\frac{N[(N+1)(N-1)(N+2)] \sigma}{\left[(N+1) p_{b}+N p_{C L}\right] r_{b}^{3}},
$$

where $r_{b}$ is the radius of the bubble, $\sigma$ is the surface tension, and $M$ the number of the harmonic. If we take $N=2$, which corresponds to the lowest critical weber number and set $f=f_{b}$, a criterion for the bubble size that escapes fragmentation can be formed. In Ref. 11, this criterion is shown to be

$$
\frac{24}{3 \frac{\rho_{b}}{\rho_{C L}}+2}=31 \frac{\rho_{C} v^{v^{\prime}}}{\sigma} \frac{r_{b}^{3 / a}}{L^{\not b}}
$$

where $v^{\prime}$ is the turbulence velocity and $L$ is the integral scale of the flow. In chamel flow, $L$ is the hydraulic diameter. For the present model, we tentatively set $L=2 r_{b}$. To verify this approach, we compare Eq. (86) with a similar expression given in Ref. 12 using a critical Weber number, Web, for bubbly flow 


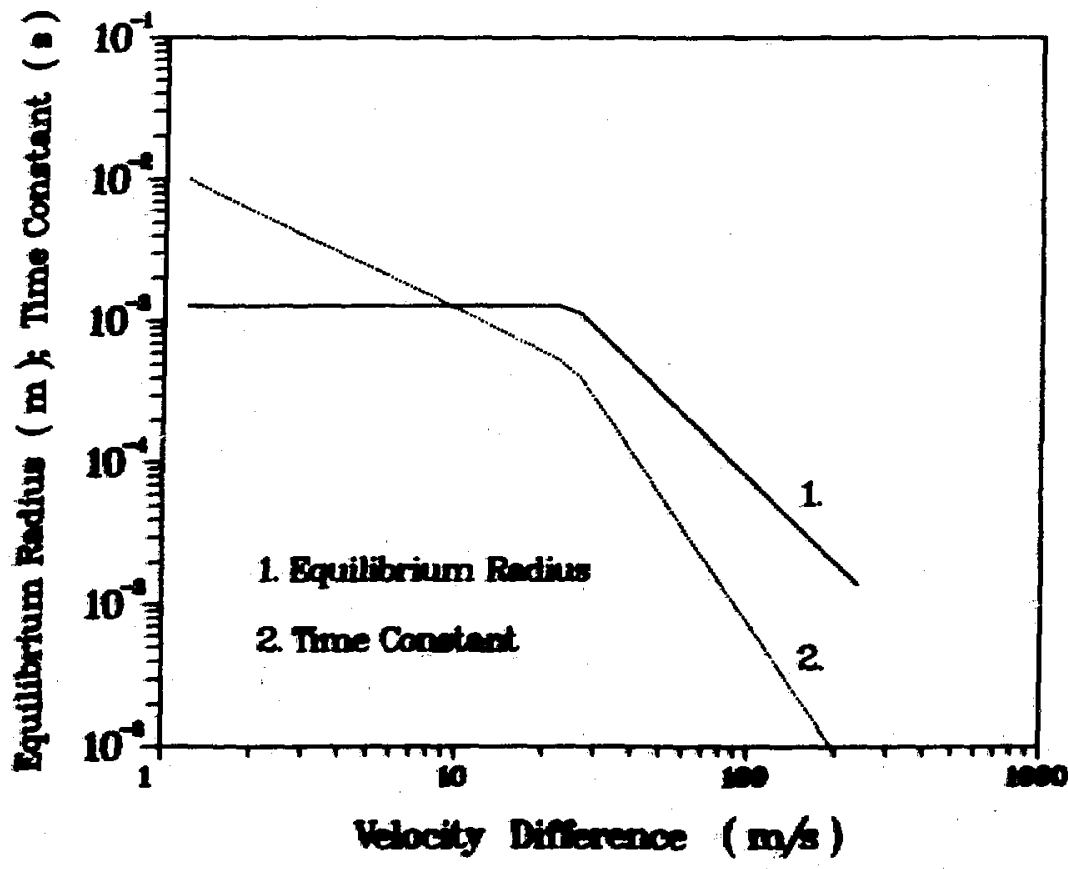

Fig. 8 .

Example of the breakup of droplets by dynamic forces.

$$
w_{b}=\frac{P_{C L} \overline{\bar{v}}^{2} 2 r_{b}}{\sigma}
$$

The root mean square turbulence velocity, $\overline{\bar{v}}$, has to be compared to $v^{\prime}$. Reference 13 gives an estimate for the turbulency velocity with

$$
v^{\prime} v^{\prime}=\left(2 r_{b} \varepsilon\right)^{k}
$$

where $\varepsilon$ is the dissipation per unit time and mass. Similarly, Ref. 11 specifies

$$
\overline{\bar{v}}^{2}=2\left(2 \varepsilon r_{b}\right)^{\not / z}
$$

By comparison of Eqs. (88) and (89), $v^{2}=2 v^{\prime} v^{\prime}$, and Eq. (87) with $\mathrm{We}_{\mathrm{b}}=2.6$ yields 


$$
\frac{P_{L C} V^{\prime} v^{\prime}}{\sigma} r_{b}=0.65
$$

For bubbly flow with vapor not to close to the critical point, $\mathrm{Pb} / \mathrm{PCL} \ll 1$, and setting $L=2 r b$. Eq. (86) yields

$$
\frac{\rho_{C L} v^{\prime} v^{\prime}}{\sigma} r_{b}=0.61
$$

which demonstrates the appropriate choice for $L$. Since AFDM does not include a turbulence model, the value of $c$ is not knom, and $v$ ' needs to be provided by a different approximation. An obvious choice would be to assess the velocity gradient of the contimuous phase and write $v^{\prime}$ as a function of the gradient. The present model, however, is only a function of the buoyancy of the bubble in the continuous liquid. Taking the buoyancy term of the correlation given in Ref. 13,

$$
v^{\prime} v^{\prime}=C_{R G}^{2} \frac{\alpha_{G}}{1-\alpha_{S}}\left(1-\frac{\alpha_{G}}{1-\alpha_{S}}\right)\left(1-\frac{\rho_{G}}{p_{C L}}\right) G r_{b}
$$

where $C_{R G}$ is a user-defined constant. During the recalculation of experiments with AFDM, we found that it was desirable to have droplets coexisting with bubbles in a cont inuous liquid cell fragmented by the same buoyancy-driven turbulence velocity. Therefore, the remaining equations are given for bubble fragmentation as well as droplet fragmentation if bubbles are present in the same cell. We denote the discontinuous bubbles or droplets with the subscript $D$. The equilibrium radius, $r_{e}$, is given by $\mathrm{Eq}$. (86) with $r_{e}=r_{b}=L / 2$, and using Eq. (92) yields

$$
r_{e}^{2}=\frac{\sigma}{\rho_{C}} \frac{24}{31} \frac{2^{*}}{3 \frac{\rho_{D}}{\rho_{C L}}+2}\left[\frac{C_{R G}^{2} \alpha_{G}}{1-\alpha_{S}}\left(1-\frac{\alpha_{G}}{1-\alpha_{S}}\right)\left(1-\frac{\rho_{G}}{\rho_{C}}\right) G\right]^{-1} .
$$


where $C_{R G}$ is a user-defined constant. A steady-state version of the code was used to recalculate boiling pool data given in Ref. 2 . The extensive numerical experiments yielded sometimes different constants than given above. With

$$
a_{e}=\frac{3 a_{0}}{r_{e}}
$$

and the new constants,

$$
a_{e}=1.4 C_{R G} \alpha_{D}\left[\frac{P_{C L} \alpha_{G} G}{\sigma\left(1-\alpha_{S}\right)}\left(3 \frac{P_{D}}{P_{C L}}+2\right)\left(1-\frac{\alpha_{G}}{1-\alpha_{S}}\right)\left(1-\frac{P_{G}}{P_{C L}}\right)\right]^{1 / 2},
$$

were CRG is still a user-defined constant. The time constant for the fragantation process is simply derived from Eq. (84). Mdjusting the constants,

$$
\tau=C_{T T} 10^{-3} r_{D}^{1.5}\left(\frac{3 p_{D}+2 \rho_{C L}}{\sigma}\right)^{0.5}
$$

where $C_{T T}$ is a user-defined constant and ${ }_{D}$ is the radius of the discontinuous phase of the last time step. The turbulence breakup model is coded in subroutine TurBul.

Figure 9 shows an example for the equilibrium radius and time constant during breakup of bubbles by turbulence through bubbles. The parameters are chasen to be similar to those in a molten $v_{2}-p o o l$, with $\alpha_{S}=0, \sigma=0.5 \mathrm{Nm}$, $P D=2 \mathrm{~kg} / \mathrm{m}^{3}$, and $P C L=8000 \mathrm{~kg} / \mathrm{m}^{3}$. The default value of the user-defined constants are used. The time constant is calculated with $r_{D}=r_{e}$. The bubble volume fraction, $\Phi=\alpha_{G}$, runs up to the limit, $\alpha_{C D}$. Of liquid-continuous flows. Buoyancy-driven turbulence breakup and dynanic forces for bubbles are interdependent splitting processes. Both processes are therefore combined to yield a common equilibrium area and a common time constant. Using Eq. (54) and setting the time derivative to zero, the equilibrium surface area of both processes can be derived similarly as that of Eq. (62). Now, the common equilibrium area, ae, is 


$$
a_{e}=\frac{\frac{a_{e 1}}{\tau_{1}}+\frac{a_{e 2}}{\tau_{2}}}{\frac{1}{\tau_{1}}+\frac{1}{\tau_{2}}},
$$

where the subscripts 1 and 2 stand for turbulence breakup and dynamic forces for buboles, respectively. The common time constant, $\tau$, is

$$
\frac{1}{\tau}=\frac{1}{\tau_{1}}+\frac{1}{\tau_{2}}
$$

Figure 10 shows an example for the common equilibrium radius, $r_{e}=3 \alpha / a_{e}$, and the time constant as defined in Eqs. (97) and (98). The parameters are chosen to be similar to those in the molten $\mid \mathrm{O}_{2}-\mathrm{pool}$, with $\alpha_{S}=0, \sigma=0.5 \mathrm{Mm}$, $P D=2 \mathrm{~kg} / \mathrm{m}^{3}$, and $P C=8000 \mathrm{~kg} / \mathrm{m}^{3}$. The default values of the user-defined constants and $=10$ are used. The time constant is calculated with $r_{D}=$ $r_{e}$. There are four sets of curves for constant bubble volume fractions, $\alpha=$ $G$.

5. Flashing of Droplets. The flashing model is used in the droplet regime. Like the nucleation model, the increase in convectible surface area is driven by the superheat of the liquid phase. Reference 14 describes the flashing of droplets with a detailed model. Unfortunately, the forms lism used depends on the history of the droplets under investigation, an approach wich cannot be used in AFDM since only the present time-step cell variables are available. Consequently, the equilibrium radius, $r_{e}$, represents the maximm size of droplets that escape fragmentation.

$$
r_{e}=\frac{2 \sigma}{\max \left(10^{-8}, P_{S a t}, \alpha-p\right)}
$$

where $\sigma$ is the surface tension between the liquid forming the droplet and the cont inuous vapor. PSat, $L$, is the saturation pressure of the liquid, and $p$ is the cell pressure. The Jacob number, Ja, is used to assess the departure from equilibrium and hence the rate at mich equilibrium is obtained as follows: 


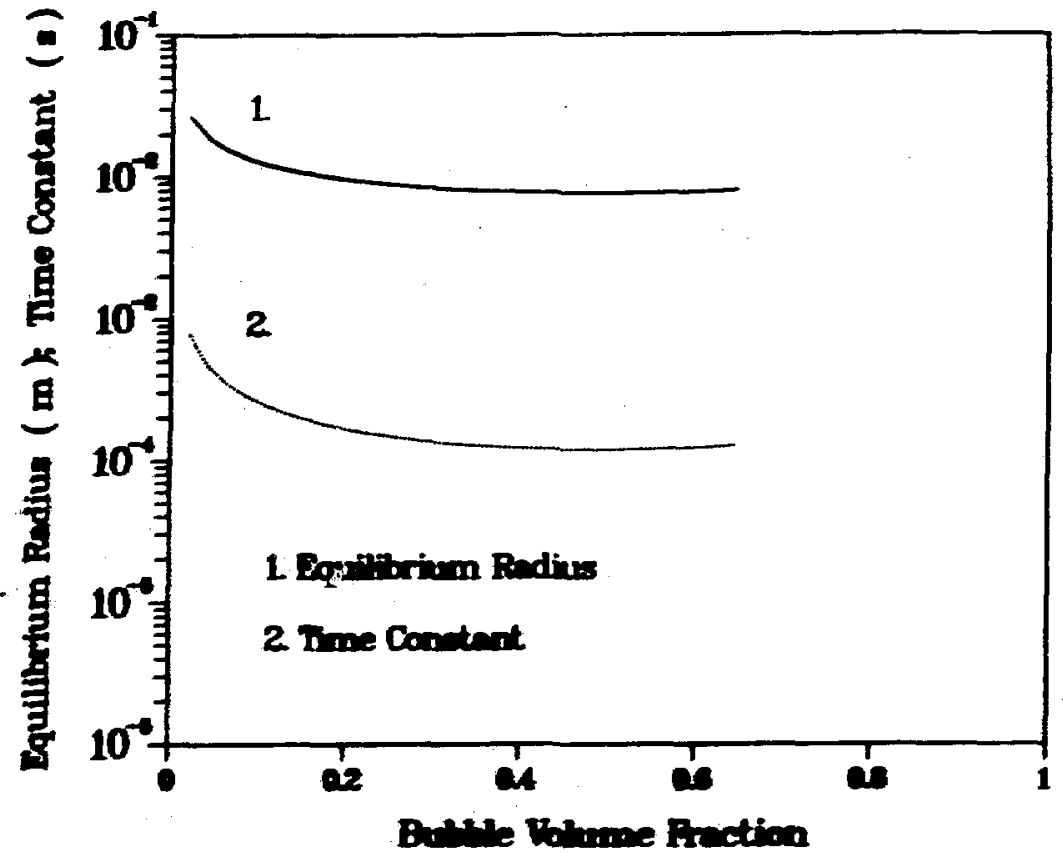

Fig. 9.

Example of the breakup of bubbles by turbulence.

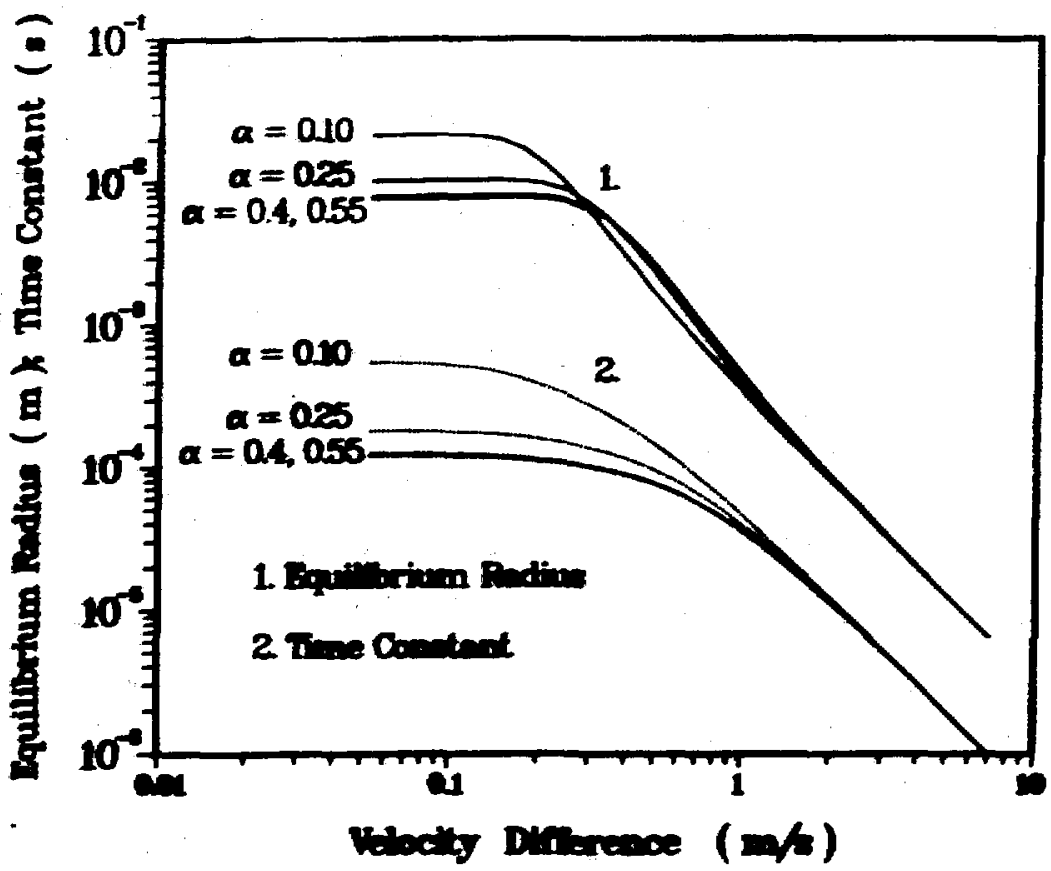

Fig. 10.

Example of bubble breakup by the combination of dynamic forces and buoyancy-driven turbulence. 


$$
J a=\frac{C_{D^{P}} D L}{P_{G} h_{1 g}} \max \left(0, T_{D L}-T_{S a t, D L}\right),
$$

where $\mathrm{CD}$ is the specific heat capacity, $\mathrm{h}_{\mathrm{Ig}}$ is the heat of vaporization, $\mathrm{T} D$ is the bulk temperature of the droplet, and TSat, DL is the saturation temperature of the liquid forming the droplet. The time constant for the fragmentation is now

$$
\tau=C_{F L} \frac{C_{D L} P L_{L} r_{d}^{2}}{k_{D L}^{J a}} \text {, }
$$

where $C_{F L}$ is a user-defined constant, $k_{D L}$ is the thermal conductivity, and $r_{d}$ is the droplet radius of the last time step. The flashing model is coded in subrout ine FLASH.

Figure 11 shows the equilibrium radius and time constant during breakup of droplets by flashing. The parameters are chosen to be similar to those in a molten $\mathrm{UO}_{2}-\mathrm{POOl}$, with $P Q L=8000 \mathrm{~kg} / \mathrm{m}^{3}, P G=2 \mathrm{~kg} / \mathrm{m}^{3}, \mathrm{CQL}=500 \mathrm{~J} /(\mathrm{kg}-\mathrm{K})$, $h_{/ g}=2 \cdot 10^{6} \mathrm{~J} / \mathrm{kg}, \mathrm{kL}=4 \mathrm{w} /(\mathrm{m}-\mathrm{K})$, and $\sigma=0.5 \mathrm{~N} / \mathrm{m}$. The default values of the user-defined constants and the simplified EOS are used. The liquid temperature, $T \mathrm{DL}$, is set to $3800 \mathrm{~K}$, with the corresponding vapor pressure, pSat, $\mathrm{DL}=$ $206661 \mathrm{~Pa}$. The saturation temperature, TSat, $\mathrm{DL}$, is calculated on the basis that only $U_{2}$-vapor is present. The time constant is calculated with $r_{d}=r_{e}$. The values are given as functions of the pressure difference, PSat, $D L-p$.

6. Coalescence. The coalescence models are used for all flow regimes except the nucleating regime. Bubbles and droplets can coalesce due to random collision. Using a simple interaction model like that of Ref. 15, the variation of the droplet or bubble radius with time yields

$$
\frac{d}{d t} r_{D}=\frac{\omega \alpha_{D} v^{\prime}}{8\left(1-\alpha_{S}\right)}
$$




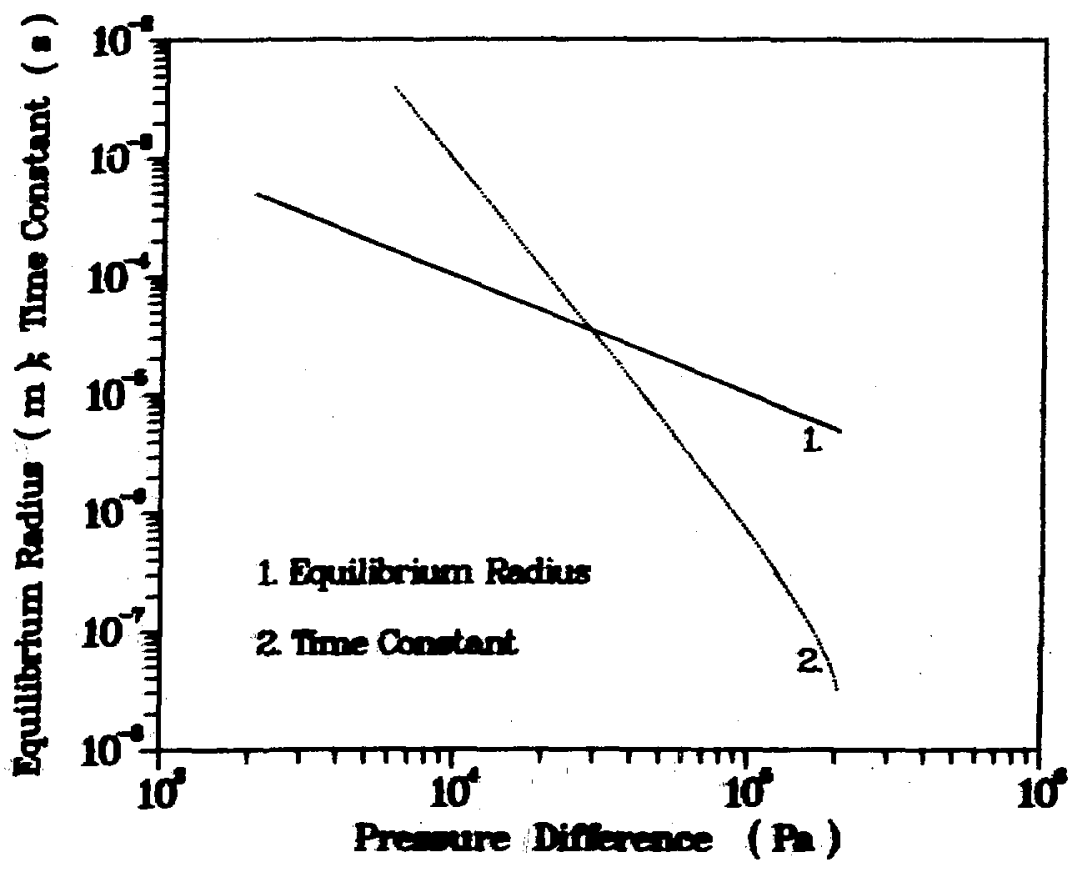

Fig. 11.

Example of the breakup of droplets by flashing.

where $r_{D}$ is the radius of the discont inuous bubble or droplet, $w$ is the coalescence probability, and $v^{\prime}$ is the velocity of the random movements of the discontinuous phase. Equation (102) shows that the derivative of ${ }_{D} D$ is given rather than the equilibrium radius and the time constant. Therefore, the coalescence rate, $I_{C}$, has to be treated differently in Eq. (54). The coalescence rate is defined using $a^{C}=3 \times 00 / r D$ and

$$
\frac{d}{d t} a^{c}=-\frac{\left(a^{c}\right)^{2}}{3 a_{D}} \frac{d}{d t}{ }^{\prime} D
$$

and substituting so that

$$
\frac{d}{d t} a^{c}=-\left(a^{c}\right)^{2} r_{c}
$$

The velocity of random movements, $v^{\prime}$, is supposed to be proportional to the velocity difference, $\Delta v$, between the discontinuous bubbles or droplets and the 
cont inuous phase. The numerical experiments with respect to the data of Ref. 2 were best fitted if the coalescence could be substantially increased with increasing discontinsous volume fraction. Especially for churn-turbulent flow, it became apparent that the decrease in momentum coupling could not be modelled by a decrease in the drag coefficient alone. We therefore increased the coalescence in such a way that the convectible surface area had a minimum at superficial velocities prevailing for buoyancy-driven churn-turbulent flow.

Eventually

$$
r_{C}=C_{C O} \frac{\Delta v}{1-\alpha_{S}} \exp \left[\max \left(0, C_{R T} \frac{\alpha_{D}}{1-\alpha_{S}}\right)\right] \text {, }
$$

where $C_{C O}$ stands for the user-defined constants $C_{C O B}$ and $C_{C O D}$ for bubbles and droplets, respectively, and $C_{R T}$ stands for $C_{R T B}$ and $C_{R T D}$ for bubbles and droplets, respectively. The coalescence model is coded in subrout ine COALES.

\section{Special Cases}

If the continuous phase changes, there can be a step change of cell variables wich govern the equation used to describe the source terms $S_{A}, f$ and $S_{B}, f$ of Eq. (53). The cell variables have the values of the end of the last time step, wich are based on the old topologies. The interfacial area is updated using $S_{A, f}$ and $S_{B}, f$ before the momentum coupling can reflect the new cell condition. For example, last-time-step velocities are still used in the source term nodels together with new topologies and a possible switch in the continuous phase. Therefore, the inconsistency at those switches is reduced by setting the source term to zero:

$$
\begin{array}{ll}
S_{A, f}=0 & \text { for } I_{T}^{n} \neq 1_{T}^{n+1} \\
S_{B, f}=0 & \text { for } 1_{T}^{n} \neq I_{T}^{n+1}
\end{array}
$$

For the source terms of the discontinuous liquid 1, a limited influence of the solid particulates has to be taken into account. The convectible surface 
area of liquid 1 will eventually be combined with the surface area of the solid particulates, as described in Sec. IV. However, no source terms are specified for the solid particulates because the present simplified approach associates a constant radius to them. By introducing a limited influence of the solid particulates, consideration is given to the fact that they share the same velocity field with liquid 1. If the volume fraction of the solid particulates, ap, is appreciably larger than that of liquid 1, a 1 , the source terms are set to zero:

$$
\begin{array}{ll}
S_{A, L 1 C P}=0 & \text { for } \alpha_{p}>C_{P A} \alpha_{L 1} \\
S_{B, L 1 C P}=0 & \text { for } \alpha_{p}>C_{P A} \alpha_{L 1},
\end{array}
$$

where LICP stands for the interface between L1 and the continuous phase, and CPM is a user-defined constant.

\section{Update Due to Mass Transfer}

After completing the heat- and mass-transfer operation in AFDM Step 1, the convectible surface areas have to be updated as a function of the change in the volume fraptions of the cell under investigation. The terms $S_{A, i}$ and $S_{B, i}$ as well as the convection term of $\mathrm{Eq}$. (52) are set zero, and the convectible surface area, $a^{C}$, is changed due to the source term $S_{C}, i$. There are two sets of regimes with different update procedures. First, in the nucleating regime, the cinvectible surface area is a function of the bubble number density and the volume fraction according to Eq. (65). Second, for all other regimes, the convectible surface area is a function of the representation radius and the volune fraction, using Eq. (1). For both sets of regines, the update equation is slightly different because of the first independent variable.

For the nucleating regime, the update equation is

$$
a^{n+1}=a^{n}+(36 \pi \tilde{n})^{\frac{1}{2}}\left[\left(\alpha^{n+1}\right)^{x}-\left(\alpha^{n}\right)^{x /}\right],
$$


where $a$ is used instead of $a^{c}$, and $\tilde{n}$ is the number density of the bubbles as calculated for the present time step.

For the other regimes, the update equation is

$$
a^{n+1}=a^{n} \frac{3}{\tilde{r}}\left(\alpha^{n+1}-\alpha^{n}\right)
$$

where $\tilde{r}$ is the representative radius for bubbles or droplets as calculated for the present time step.

In analogy to Eqs. (63) and (64), the updated surface area is limited by the user-defined constants as given for these equations. For droplets,

$$
a^{n+1}=\max \left[\frac{3 \alpha_{d}}{r_{x d}}, \min \left(\frac{3 \alpha_{d}}{r_{m d}}, a^{n}+\Delta a\right)\right],
$$

where $\alpha_{d}$ is the droplet volume fraction and $\Delta a$ is given by Eq. (109), and for bubbles,

$$
a^{n+1}=\max \left[\frac{3 \alpha_{b}}{r_{x b}}, \min \left(\frac{3 \alpha_{b}}{r_{m b}}, a^{n}+\Delta a\right)\right],
$$

where $o_{b}$ is the bubble volume fraction and $\Delta a$ is given by either Eq. (109) or (108).

In case that the mass transfer yields volume fractions that were not present before in the cell under investigation, the newly created bubbles or droplets need to have a surface area associated to them. For droplets, the new radius, $r d$, is

$$
r_{d}=\max \left[r_{m d},\left(\frac{3 \alpha_{d}}{4 \pi\left(1-\alpha_{S}\right) n_{\text {DROP }}}\right)^{3 /}\right],
$$


when $\alpha_{d}$ is the newly created volume fraction of droplets, and noROP is a userdefined number density of droplets. Now

$$
a^{n+1}=\max \left(\frac{3 \alpha_{d}}{r_{x d}}, \frac{3 \alpha_{d}}{r_{d}}\right)
$$

For bubbles, we take the nucleation model and Eq. (68) for all liquids present in the cells, and evaluate the number density, $n_{b}$, of bubbles based on the actual cell variables. The radius, $r_{b}$, of newly created bubbles is therefore

$$
r_{b}=\max \left[r_{m b},\left(\frac{3 a_{b}}{4 \pi \sum_{m} n_{b m}}\right)^{3 / 2}\right],
$$

where $\alpha_{b}$ is the total vapor volume fraction, and $n_{b m}$ is the number density in liquid component $\mathrm{m}$. Now

$$
a^{n+1}=\max \left(\frac{3 a_{b}}{r_{x b}}, \frac{3 a_{b}}{r_{b}}\right)
$$

where the limiting radius, $r_{x b}$, is usually dominated by $n_{\min }$ of Eq. (68).

IV.

Knowing the convectible surface areas in a cell, and the volume fractions of the components present, the interfacial surface areas between all components can be assessed with models independent of time. Therefore, these surface areas are called instantaneous interfacial areas. They are subdivisions of the convectible surface areas and the structure surface area, as, which is an input constant. Table 11 shows, for a given topology, the components or partners between which an instantaneous interfacial area is defined. Note that, if solid 
particulates, $P$, are present, they share a common surface with liquid 1 if both form the discontinuous phase, or are totally surrounded by liquid 1 if liquid 1 is the continuous phase. In the latter case, they can only have an instantaneous interfacial area with either liquid 1 or the structure. Because of the bulk models for heat and mass transfer, it is sufficient to have the energy from any discontinuous component exchanged with the solid particulates via liquid 1. The topologies 4,7 , and 11 have therefore fewer areas specified in Table 11.

There are five subdivision criteria which will be outlined in the next sections. With the basic model approaches chosen, the instantaneous interfacial areas will then be specified for each topology. The combinations of the different models impose a complicated logic on the computational procedure, as described in Sec. IV.K.

\section{A. Structure Contact}

The surface area, as, of the structure component is given as an input constant. The structure of the present AFDM models simply represents a volume fraction not available to fluid flow with limited influence on the hydrodynamics of the flow. Although energy and momentum are allowed to be exchanged, there is no model available to treat the influence of the structure on velocity, temperature, and volume fraction profiles inside the fluids, as would be necessary for modelling channel flow. Therefore, the subdivision of as is simply based on volume fraction relationships with

$$
a_{D S}=\frac{1}{2} \frac{\alpha_{D}}{1-\alpha_{S}} \min \left(a_{D}^{c}, a_{S}\right)
$$

where aDS is the instantaneous interfacial area between the discontinuous component and the structure, and $a_{D}^{c}$ is the convectible surface area of the discontinuous component. The left-over surface area is associated to the continuous phase

$$
{ }^{a_{C S}}=a_{S}-\sum a_{D S}
$$




\begin{tabular}{|c|c|c|c|c|c|c|c|c|c|c|c|}
\hline \multirow{2}{*}{$\frac{\text { Topology }}{1}$} & \multirow[t]{2}{*}{$\begin{array}{l}\text { Convectible } \\
\text { Surface Area } \\
\text { Partners } \\
\end{array}$} & \multicolumn{4}{|c|}{$\begin{array}{c}\text { Instantaneous } \\
\text { Interfacial Area } \\
\text { Partners } \\
\end{array}$} & & & & & & \\
\hline & & $S G$ & & & & & & & & & \\
\hline 2 & & SP & SL1 & PL1 & & & & & & & \\
\hline 3 & & SL2 & & & & & & & & & \\
\hline 4 & L1L2 & SP & SL1 & SL2 & PL1 & L1L2 & & & & & \\
\hline 5 & L1L2 & SP & SL1 & SL2 & PL1 & PL2 & L1L2 & & & & \\
\hline 6 & GL1 & $S P$ & SL1 & SG & PL1 & $P G$ & $\mathrm{~L} 16$ & & & & \\
\hline 7 & GL1 & SP & SL1 & SG & PL1 & L1G & & & & & \\
\hline 8 & GL2 & SL2 & SG & L2G & & & & & & & \\
\hline 9 & G2 & $\mathrm{SL2}$ & SG & L2G & & & & & & & \\
\hline 10 & $G 1, G 2$ & SP & SL1 & SL2 & SG & PL1 & PL2 & $P G$ & L1L2 & $\mathrm{L} 1 \mathrm{G}$ & L2G \\
\hline 11 & L1L2, GL1 & SP & SL1 & SL2 & SG & $P L 1$ & L1L2 & L1G & L26 & & \\
\hline 12 & L1L2, GL2 & SP & SL1 & SL2 & SG & PL1 & PL2 & PG & L1L2 & LIG & L2G \\
\hline
\end{tabular}

Note: The order of the partners of the instantaneous interfacial area can be exchanged.

where acs is the instantaneous interfacial area between the continuous phase and the structure, and the sum is over all discontinuous components in the cell, including the solid particulates.

\section{B. Liquid 1 - Solid Particulate Contact}

Liquid 1 and solid particulates share the same velocity field. If liquid 1 is the continuous phase, the solid particulates are assumed to be immersed in liquid 1 and can share an instantaneous interfacial area only with the structure and liquid 1 . If liquid 1 is a discont inuous component, the contact criteria depend on the volume fractions, sizes, and interfacial conditions 
of both liquid 1 and solid particulates. The model presented here is kept as simple as possible. It was desirable to (a) model a non-exclusive contact between both partners, and both partners and other components, and (b) assess whether melting or freezing occurs based on instantaneous contact temperatures.

To describe the contact of liquid 1 and solid particulates with the other components if liquid 1 is discontinuous, a fluid particle of the combined liquid 1 and solid particulate volume fraction is introduced. Knowing the radius, $r_{p}$, of the particulate wich is an input constant, and the radius, $r_{L 1}$, of the liquid 1 fluid particles through

$$
r_{L 1}=\max \left(r_{m d}, \frac{3 \alpha_{L 1}}{a_{L 1 C P}^{c}}\right),
$$

where $a_{L 1 C P}^{c}$ is the convectible surface area between liquid 1 and the cont inuous phase, the common radius, $\mathrm{Cm}$, of the combined liquid 1-particulate components is based on a volume average with

$$
r_{c M}=\left(\alpha_{p}+\alpha_{L 1}\right)^{1 / 3}\left(\frac{\alpha_{p}}{r_{p}^{3}}+\frac{\alpha_{L 1}}{r_{L 1}^{3}}\right)^{-1 / 2} .
$$

The surface area associated to this radius is

$$
a_{C M}=\frac{3\left(\alpha_{p}+\alpha_{L 1}\right)}{{ }^{r}{ }_{C M}} .
$$

Figure 12 shows, for a given constant $\alpha_{p}+\alpha_{L 1}=0.2$, the value for $r_{c u}$ and acu as a function of the relative liquid 1 volume fraction. The averaging procedure yields surface areas wich are close to the larger value for most of the parameter range.

To assess the subdivision of acm and the contact between liquid 1 and solid particulates, if liquid 1 is discontinuous, two conditions will be introduced. They are based on whether the instantaneous interface temperature between liquid 1 and particulate is above or below the melting temperature. Only 
the bulk temperatures for liquid $1, T_{L 1}$, and for solid particulates, $T_{p}$, are known. The instantaneous contact temperature, $T_{1 C}$, is based on the Fourier equation with

$$
T_{I C}=\frac{\left(k_{p} c_{p} p_{p}\right)^{1 / /} T_{p}+\left(k_{L 1} c_{L 1} p_{L 1}\right)^{1 / 2} T_{L 1}}{\left(k_{p} c_{p} p_{p}\right)^{3 / 2}+\left(k_{L 1} c_{L 1} \rho_{L 1}\right)^{1 / 2}},
$$

where $k$ is the thermal conductivity, $c$ is specific heat, and $p$ the microscopic density. To take into account vanishing voiume fractions, an effective contact temperature. TEC, is calculated with

$$
\begin{aligned}
& T_{E C}=\left(1-10 \frac{\alpha_{p}}{\alpha_{p}+\alpha_{L 1}}\right) T_{L 1}+10 \frac{\alpha_{p}}{\alpha_{p}+\alpha_{L 1}} T_{I C} \text { for } \frac{\alpha_{p}}{\alpha_{p}+\alpha_{L 1}} \leqq 0.1 \\
& T_{E C}=T_{1 C} \quad \text { for } 0.1<\frac{\alpha_{p}}{\alpha_{p}+\alpha_{L 1}}<0.9 \\
& T_{E C}=\left(1-10 \frac{\alpha_{L 1}}{\alpha_{p}+\alpha_{L 1}}\right) T_{p}+10 \frac{\alpha_{L 1}}{\alpha_{p}+\alpha_{L 1}} T_{I C} \text { for } \frac{\alpha_{p}}{\alpha_{p}+\alpha_{L 1}} \geqq 0.9 .
\end{aligned}
$$

The effective contact temperature is compared to the melting temperature, $T_{M I t, L 1}$, of component 1 . The melting-freezing indicator, IMF, is used to transfer the condition to the surface area subdivision models and the model for defining the drag coefficient; see Vol. III, Sec. III.D.5.

$$
\begin{array}{ll}
I_{M F}=1 & \text { for } T_{E C}<C_{M T} T_{M I t, L 1} \\
I_{M F}=0 & \text { for } T_{E C} \geqq C_{M T} T_{M I t, L 1},
\end{array}
$$




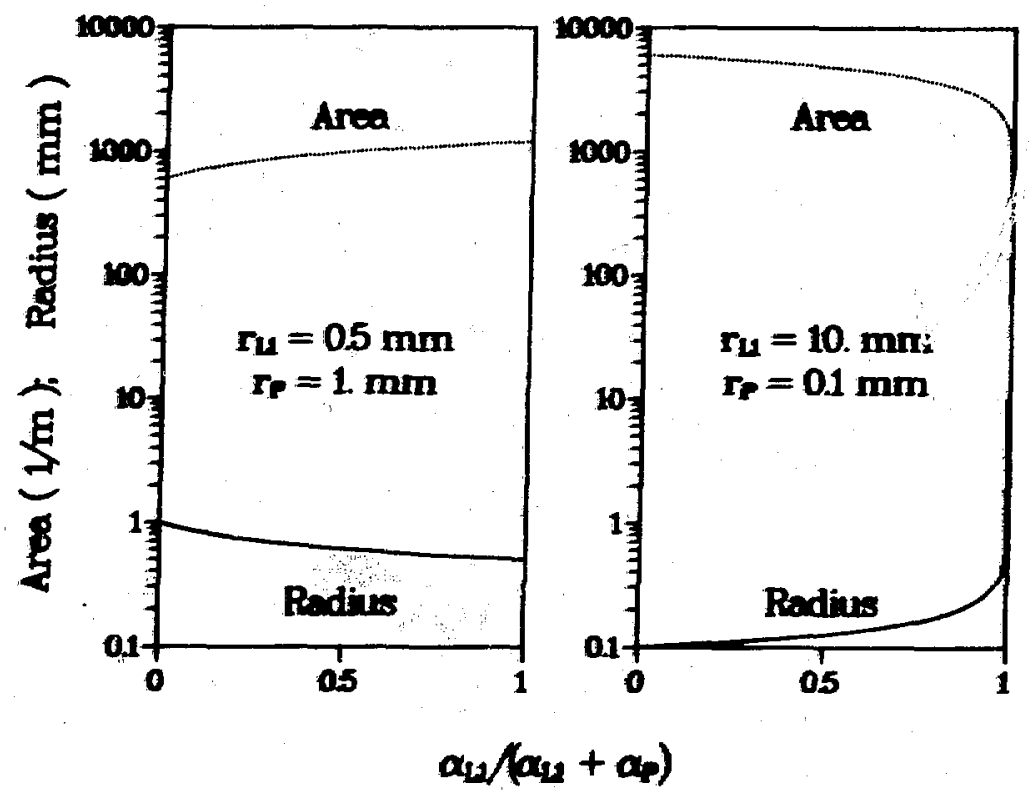

Fig. 12.

Values for the comon radius and interfacial area of the particle-liquid 1 system.

where $C_{\text {MIT }}$ is a user-defined constant. This calculation is performed in subroutine TEFFEC.

After having defined the melting-freezing indicator a choice is being made of wich component, liquid 1 or particulates, occupies most of the outside surface. Four dimensionless functions of the volume fractions are introduced, and limiters are set to describe the subdivis $n$.

$$
\begin{aligned}
& c^{* *}=\min \left(1, \frac{\alpha_{L 1}}{\left(\alpha_{p}+\alpha_{L 1}\right) \alpha_{L M P}}\right) \\
& C^{++}=\min \left(1, \frac{\alpha_{p}}{\left(\alpha_{p}+\alpha_{L 1}\right)_{\alpha_{L P}}}\right),
\end{aligned}
$$

where aup is a user-defined constant. Figure 13 shows the two functions above in the range of $\alpha_{L 1}$ or $\alpha_{p}$ were they are not constant. The present default 
value for aup is used. The choice of the topology, and therefore of the presence of components, is based on whether the sum of $\alpha_{L} 1$ and $\alpha_{p}$ vanishes or not. Consequently, the functions of Eqs. (124) and (125) are used to force the influence of liquid 1 to zero if a 1 disappears, and to force the influence of the particulates to zero if $\alpha_{p}$ disappears.

The choice of the remaining dimensionless functions is made on whether the melting-freezing indicator, IMF is zero or one

$$
\begin{array}{ll}
C^{*}=1-\left(1-C_{P C P}\right) C^{* *} & \text { for } I_{M F}=0 \\
C^{*}=1-C_{L C P} C^{* *} & \text { for } I_{M F}=1,
\end{array}
$$

where $C_{P C P}$ and $C_{L C P}$ are user-def ined constants.

$$
\begin{array}{ll}
C^{+}=1-C_{P C P} C^{++} & \text {for } I_{M F}=0 \\
C^{+}=1-\left(1-C_{L C P}\right) C^{++} & \text {for } I_{M P_{P}}=1 .
\end{array}
$$

If $h_{F}=0$, then liquid 1 is predominantly on the outside of the common L1-P fluid particle; if $I M F=1$, then the same is true for the particulates. Now, $\mathrm{C}^{+}$is used to describe the liquid 1 contacts, mereas $\mathrm{C}^{*}$ is used to describe the contacts of the particulates. Figure 14 shoms an idealized configuration for $h_{w F}=0$ or 1 (if the numbers of droplets and solid particulates are equal). The sketch is to show that the constants CPCP or CLCP represent that fraction of the common surface that the inner component shares with the continuous phase. The constant CPCP describes the residual particle contact, and $C_{L C P}$ describes the residual liquid 1 contact to the continuous phase. If Inf $=1$, then most of the common surface is occupied by the solid particulates; if IMF $=0$, then liquid 1 occupies most of the common surface.

For vapor-continuous flows, there is an additional choice available to describe the liquid 1 particulate contact. If the user-defined constant, ILPC, is set to zero, the contact in vapor-continuous flow is the same as in liquidcont inuous flow. If ILPC is set to one, liquid 1 droplets and solid particulates are supposed to be separated from each other in vapor-continuous flows. 


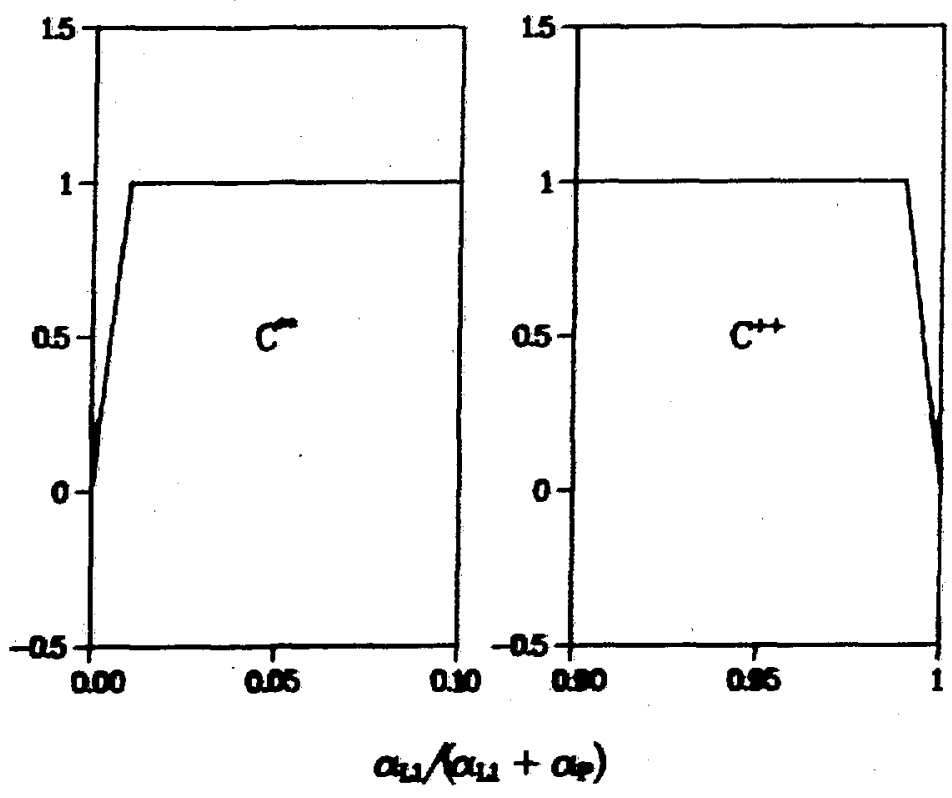

Fig. 13.

Particle-liquid 1 dimensionless functions describing interfacial area contact.

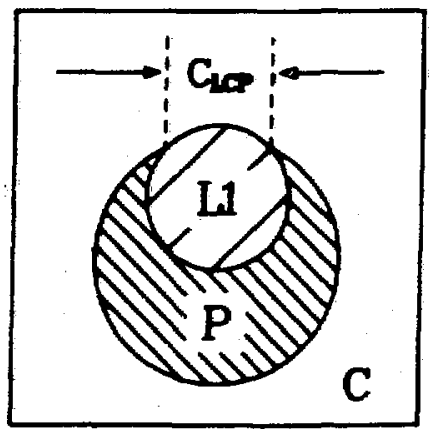

It $=1$

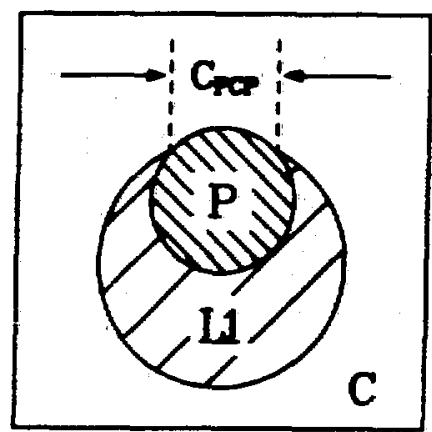

$\mathrm{I}=0$

C Contimuous Phase

P Solid Particulates

\section{L1 Liquid 1}

Fig. 14

Idealized particle-liquid 1 configurations based on IMF $=1$ (freezing) or I $_{\mathrm{w}}=0$ (melting). 
and their contact is described by the interaction model of Sec. IV.C, since the collision contact is supposed to be independent of the velocities.

Eventually, the contact between liquid 1 and particulates is calculated first, and then the contact bet wen of them to the continuous phase.

$$
\begin{gathered}
a_{P L 1}=C_{L 1 P} C^{* \star} c^{++} a_{C M} \quad \text { for } I_{L P C}=0 \text { or } I_{\phi}=0 \\
a_{P L 1}=\min \left[\frac{3 \alpha_{p}}{2 r_{p}}, \frac{a_{L 1 C P}^{c}}{2}, C_{P L 1} \frac{\alpha_{L 1} \alpha_{p}}{1-\alpha_{S}} \frac{\left(r_{L 1}+r_{p}\right)\left(r_{L 1}^{2}+r_{p}^{2}\right)}{r_{L 1} r_{P} \max \left(r_{L 1}^{2}, r_{p}^{2}\right)}\right] \\
\text { for } I_{L P C}=1 \text { and } I_{\phi}=1,
\end{gathered}
$$

where $r_{p}$ is the user-defined radius of the solid particulates, $Q_{L 1 P}$ and $C_{P L 1}$ are user-defined constants, a $\mathrm{CM}$ is the common surface area of Eq. (120), and 14 is the continuous phase indicator of Eqs. (27) and (28). For ILPC $=0$, the common surface area is subdivided using the dimensionless functions

$$
\begin{aligned}
& { }^{a_{P C P}}=C^{*} C^{++}{ }^{a} \mathrm{Cn} \text { for } \mathrm{I}_{L P C}=0 \text { or } \mathrm{I}_{\phi}=0 \\
& a_{L T C P}=C^{+} C^{* \star} a_{C M} \quad \text { for } L_{L P C}=0 \text { or } 1_{1}=0 \text {. }
\end{aligned}
$$

if liquid 1 and particulates are supposed to be separated, ILCP $=1$, and the individual surface areas are

$$
\begin{aligned}
& a_{P C P}=\frac{3 \alpha_{p}}{r_{p}}-a_{L 1 P} \quad \text { for } L_{L P C}=1 \text { and } I_{\phi}=1 \\
& a_{L 1 C P}=a_{L 1 C P}^{c}-a_{L 1 P} \text { for } L_{L P C}=1 \text { and } I_{\phi}=1 .
\end{aligned}
$$

53 


\section{Collision Contact}

In liquid and vapor continuous flows, two discontinuous components, irrespective whether they are bubbles or droplets, can share a surface area if they collide. This contact area has to be a fraction of the convectible surface areas and limiters have to be applied. To assess the contact area, the approach of Ref. 15 is used. The two discontinuous components have average random velocities, $\bar{v}_{1}$ and $\bar{v}_{2}$, wich may be different from the velocities of the momentum equation. There are $n$ discontinuous spheres in a cell, with

$$
n=\frac{3 \alpha}{4 \pi r^{3}}
$$

The volume swept per unit time and unit volume by all spheres of one component is

$$
v^{*}=\frac{3 \alpha}{4 \pi r^{3}} \pi r^{2} \bar{v}
$$

Multiplying this volume with the number of spheres of the second discontinuous component yields the collision rate when the first component moves. The total collision rate, $n^{\star}$, is approximately the algebraic average of both collisions, the rate when component 1 moves, and the rate when component 2 moves.

$$
n^{*}=\frac{0.5}{1-\alpha_{S}} \frac{3 \alpha_{1}}{4 \pi r_{1}^{3}} \frac{3 \alpha_{2}}{4 \pi r_{2}^{3}}\left(\pi r_{1}^{2} \bar{v}_{1}+\pi r_{2}^{2} \vec{v}_{2}\right)
$$

The surface area during the collision, $A_{12}$, is supposed to be the cross-section area of the smaller droplet

$$
A_{12}=\pi \min \left(r_{1}^{2}, r_{2}^{2}\right)
$$

The time, $t_{12}$, that the contact lasts is about the time to travel the distance $2 r_{1}+2 r_{2}$ with an average velocity, $\bar{v}_{a}$. 


$$
t_{12}=\frac{2\left(r_{1}+r_{2}\right)}{\bar{v}_{a} / 2}
$$

The average velocity is divided by two in order to account for the fact that the velocity vector of both components may have the same direction. The total contact area per unit volume, $\mathrm{a}_{\mathrm{DD}}^{V}$, of all collisions of both discontinuous components is the product of the quantities above, with

$$
{ }_{D D}^{v}=n^{*} A_{12} t_{12}=C_{p p} \frac{\alpha_{1} \alpha_{2}}{1-\alpha_{S}} \frac{\left(r_{1}+r_{2}\right)\left(r_{1}^{2} \bar{v}_{1}+r_{2}^{2} \bar{v}_{2}\right)}{r_{1} r_{2} \max \left(r_{1}^{2}, r_{2}^{2}\right) \bar{v}_{a}},
$$

where $C_{p p}$ is a user-defined constant. If no correction factors are used in conjunction with these equations, $C_{p p}=9 / 8$. To simplify the correlation considerably, all random velocities are set equal. Therefore, the contact area is no longer a function of the velocities but only of the volume fractions and radi i, with

$$
a_{D D}^{v}=C_{p p} \frac{\alpha_{1} a_{2}}{1-\alpha_{S}} \frac{\left(r_{1}+r_{2}\right)\left(r_{1}^{2}+r_{2}^{2}\right)}{r_{1} r_{2} \max \left(r_{1}^{2}, r_{2}^{2}\right)} \text {. }
$$

The contact area is limited so that it cannot exceed half of the convectible surface area reduced by the contact with the structure.

\section{Persisting Contact}

In liquid-continuous flows, bubbles and droplets can share a surface area even if they have the same velocity. They are supposed to be in a persisting contact which is governed only by the surface conditions. This contact area has to be a fraction of the convectible surface area, and Iimiters have to be applied. Reference 16 presents a method to predict three component contacts for topologies 11 and 12 . The sum of surface tensions, $S_{A}$ and $S_{B}$, are

$$
S_{A}=\sigma_{G C L}-\sigma_{G D L}-\sigma_{L L}
$$


and

$$
S_{B}=\sigma_{G O L}-\sigma_{G C L}-\sigma_{L L}
$$

Depending on the values of $S_{A}$ and $S_{B}$, there are three different configurations, wich are show in Fig. 15. In the first configuration, the bubble of $r_{b}<r_{d}$ is inside the droplet. A residual surface area is provided for the contact between vapor and the continuous phase by the user-defined constant $C_{C L G}$ which defines the fraction of the surface available. The second configuration is defined by dividing the smaller convectible surface ( $F i g .15: r_{b}<r_{d}, a_{b}<a_{d}$ ) into two equal parts for the contact between the discontinuous and the cont inuous component. This is a rough approximation of the complicated contacts depending upon the contact angles. In the third configuration, the bubble has almost no contact to the droplet. However, a user-defined constant, CDLG, is used to avoid the exclusiveness of this contact by defining a residual contact area. The fractions. $B$, of the available convectible surface area are, in the order of the three configurations, 1, 2, 3

$$
\begin{array}{ll}
B=1-C_{C L G} & \text { for } S_{A} \geqq 0 \text { and } S_{B}<0 \\
B=0.5 & \text { for } S_{A}<0 \text { and } S_{B}<0 \\
B=C_{D A . G} & \text { for } S_{A}<0 \text { and } S_{B} \geqq 0,
\end{array}
$$

where

$$
\mathrm{C}_{\mathrm{CLG}}<0.45, \quad \mathrm{C}_{\mathrm{DLG}}<0.45 .
$$

Figure G-2 of Vol. VI shows the sum of the surface tensions for the $10_{2}-$ stainless-steel system. The first value corresponds to $S_{A}$, the second to $S_{B}$, in topology 11, and vice versa in topology 12 . There are five regions of equal contact criteria for the given range of the temperature. Below $2822 \mathrm{~K}$, between $3202 \mathrm{~K}$ and $3576 \mathrm{~K}$, and above $6747 \mathrm{~K}$, configuration 2 is suggested with 
$B=0.5$, between $2822 \mathrm{~K}$ and $3202 \mathrm{~K}, \mathrm{~B}=1-\mathrm{C}_{\mathrm{CLG}}$ for topology 11 and $\mathrm{B}=\mathrm{CDLG}_{\mathrm{O}}$ for topology 12, and petween $3576 \mathrm{~K}$ and $6747 \mathrm{~K}, \mathrm{~B}=\mathrm{CDLG}_{\mathrm{Q}}$ for topology 11 and $B=1-C_{C L G}$ for topology 12.

The contact area, $a^{\sigma}$, between the two discontimuous components due to the surface tensions is now

$$
a_{G D L}^{\sigma}=B a^{c} .
$$

Reiterating the range of values for $B$ in Eqs. (142) and (143), configuration 1 of Fig. 15 prevails for $0.55<B \leq 1$, configuration 2 for $B=0.5$, and configuration 3 for $0 \leq B<0.45$.

The choice of $\mathrm{a}^{\mathrm{C}}$ in Eq. (142) depends on the radi $i$ of the discontinuous spheres, the number of spheres and some assumptions about limiting conditions. The first ! imit is necessary for topology 12 and if sol id particulates are present. The value of $B$ has to be limited as a function of the fraction of particulates in the liquid 1-particulate field because bubbles cannot be inside the solid particulates.

$$
\begin{aligned}
& B=\min \left(1-C_{C L G}, \max \left[0.5,1-C_{C L G}-0.5\left(1.15-\frac{\alpha_{L 1}}{\left(\alpha_{p}+\alpha_{L 1}\right)_{\alpha_{L P}}}\right)\right]\right) \\
& \quad \text { for } \mathrm{I}_{\mathrm{J}}=12 \text { and } \mathrm{S}_{\mathrm{A}} \geq 0 \text { and } \mathrm{S}_{\mathrm{B}}<0,
\end{aligned}
$$

where aup is a user-defined constant as in Eq. (124). Figure 16 shows the value of $B$ from Eq. (145) as a function of the volume fractions. As the volume fraction $\alpha_{L 1}$ becomes small compared to $\alpha_{p}$, the value of $B$ is forced below the threshold of 0.55 so that configuration 2 represents the contact.

The limits for the second case are those applied for bubbles with radi $i$ greater than the droplet radii. For configuration 2 and 3 , i.e., $B \leq 0.55$, the value of $\mathrm{aC}^{\mathrm{C}}$ is taken from the smaller fluid sphere of the droplet, especially because $B=0.5$ is supposed to divide the smaller sphere into two equal parts; see Fig. 15. However, for $B<0.55$, configuration 1 predicts almost total engulfing of the vapor bubble by the discontinuous liquid. If $r_{b}>r_{d}$, then the 


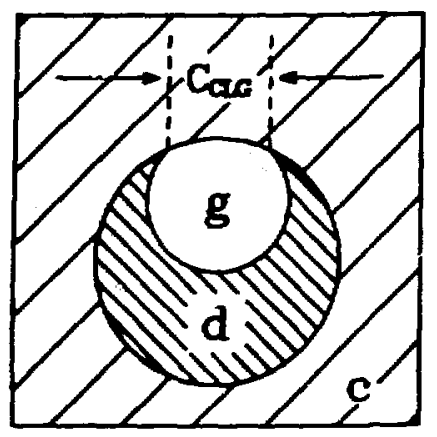

Configuration 1

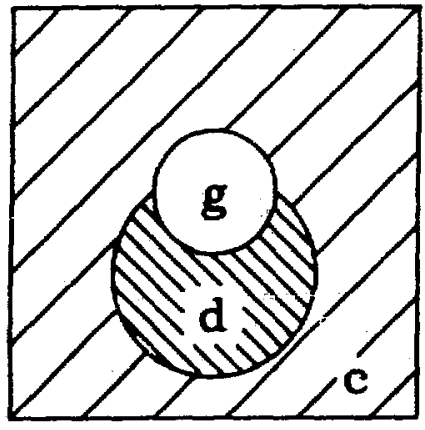

Configuration 2

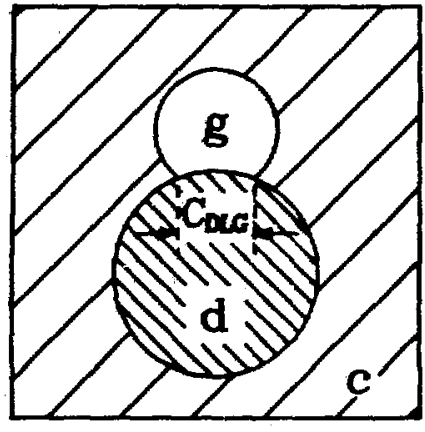

Configuration 3

c Continuous liquid

d Discontinuous liquid

g Vapor butbble

Fig. 15.

Compound bubble configurations modeled by AFDM.

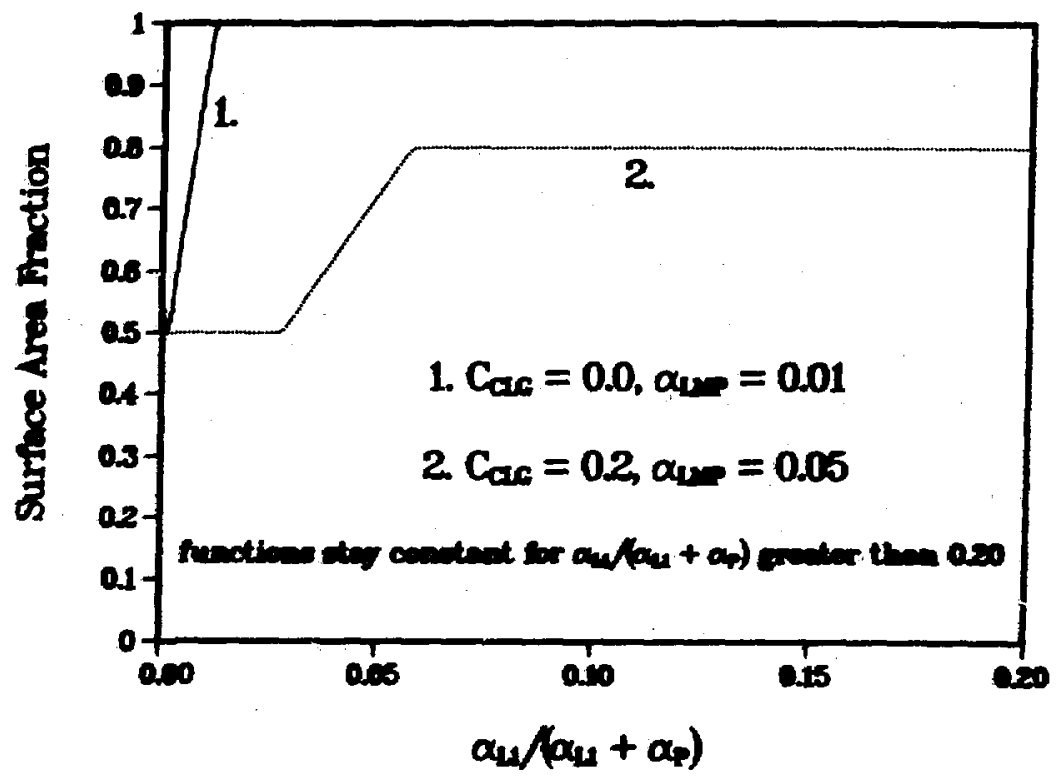

Fig. 16.

Contact fraction B given by Eq. (145). 
discontinuous liquid wraps around the bubble. Ignoring the effect on the liquid-liquid interface, the value of $a^{c}$ is taken from the bubble. By this simplification the instantaneous interfacial area between bubbles and droplets is approximated correctly, and the liquid-liquid area change is neglected. For most problems, the value of $a^{\sigma}$ will be corrected by the value of $a^{V}$ (see Sec. IV.E.), so that this distortion is less pronounced. The third limit is introduced if the number of the bubbles is considerably different from that of the droplets, and pairs cannot be formed to match the simplified picture of Fig. 15. Therefore, the number of bubbles and droplets is calculated, using

$$
\frac{n_{b}}{n_{d}}=\left(\frac{r_{d}}{r_{b}}\right)^{2} \frac{a_{G}^{c}}{a_{D L}^{c}} .
$$

The approach is to 1 imit $a^{\sigma}$ proportional to $n_{b} / n_{d}$ or its inverse if the number of the fluid spheres which are to be used to provide $\mathrm{a}^{\mathrm{C}}$ is greater than that of the other discont inuous component.

Figure 17 shows the second and third limits applied for configuration 1 (see Fig. 15). The value of $a^{c}$ is taken from the vapor bubble convectible area, ${ }_{G C L}^{C}$. The droplet that wraps around the bubble is distorted but the distortion is only accounted for by the bubble-droplet area. If $n_{b}>n_{d}$, then foc has to be multiplied by $n_{b} / n_{d}$ because not all bubbles have a droplet partner. If $n_{b}<n_{d}$, there is at least one droplet per bubble, so no limits apply.

Finally, all conditions applied

$$
\begin{aligned}
& a_{G O L}^{\sigma}=B a_{G C L}^{c} \min \left(1, \frac{n_{d}}{n_{b}}\right) \text { for } r_{b}<r_{d} \\
& a_{G D L}^{\sigma}=B a_{G C L}^{c} \min \left(1, \frac{n_{d}}{n_{b}}\right) \text { for } r_{b} \geq r_{d} \text { and } B>0.55 \\
& a_{G C L}^{\sigma}=B a_{C L D L}^{c} \min \left(1, \frac{n_{b}}{n_{d}}\right) \text { for } r_{b} \geq r_{d} \text { and } B \leqq 0.55 .
\end{aligned}
$$


If the droplets consist of liquid 1, and if solid particulates are present, acm is used instead of $a^{c}$, and $n_{d}$ is calculated as a function of $r a n$ and acu.

\section{E. Combination of Persisting and Collision Contact}

The contact areas $a^{V}$ and $a^{\sigma}$ of the two previous sections have to be combined in topologies 11 and 12. Both contact models apply for the same area between the bubbles and the droplets. A linear function is used to define the relative influence of each model. The basic condition is that at zero velocity difference between bubbles and droplets, the influence of the collision model can be neglected. Therefore, a weighting factor, MPV, is introduced as

$$
W_{P V}=\min \left(0.9, \max \left[C_{P V M}, \frac{\vec{v}_{G}-\vec{v}_{D L} \mid \Delta t C_{P V}}{2 \max \left(r_{b}, r_{d}\right)}\right]\right) .
$$

where $\vec{v}_{G}$ and $\vec{v}_{D L}$ are the velocities of the bubbles and droplets, respectively, $\Delta t$ is the time step size, and CPV and CPW are user-defined constants. Finally, for topologies 11 and 12, the contact area between bubbles and droplets is

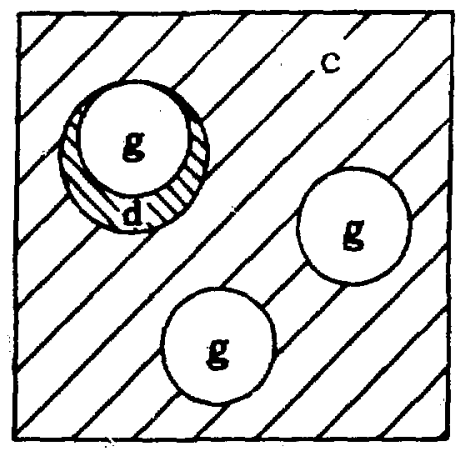

$\mathbf{n}_{\mathbf{b}}>\mathbf{n}$

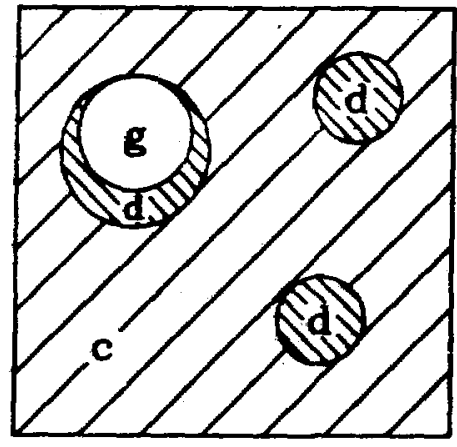

$\mathbf{n}_{\mathbf{b}}<\mathbf{n} \mathbf{d}$

\section{Configuration 1 for $r_{b} \geqq r_{d}$}

c Continuous liquid

d Discontinuous liquid

g Vepor bubbles

Fig. 17.

AFD modeling of configuration 1 the number of bubbles differs from the number of drops and $r_{b} \geq r_{d}$. 


$$
a_{G D L}^{P}=w_{P V} a_{G O L}^{V}-\left(1+w_{P V}\right) a_{G D L}^{\sigma}
$$

The superscript $P$ denotes the combined bubble-droplet contact in topologies 11 and 12. Figure 18 shows WPV as a function of the absolute velocity difference with the default values $C_{P V}=1, C_{P Y M}=0.2$, and two combinations for $\Delta t$ and $r$ :

\section{F. Cor rection Due to Aucleation}

For topologies 11 and 12, the contact area of the bubbles may change due to nucleation because bubbles can be formed in either liquid phase. To insure that the nucleating liquid shares enough surface area with the vapor phase, the bubble contact area subdivision is redirected if the conditions are such that the nucleating regime has been selected. For this regime, the other contact models are less effective because the bubbles are supposed to act like rigid spheres, and effective contact to the droplets is substantially reduced. As conditions change to non-nucleating regimes, the nucleation correction has to fade out. A weighting factor, $W_{N J}$, is introduced depending on how far away the conditions are from the nucleating regime, as defined in Sec. II.D.1.

$$
W_{N U}=\max \left(0, C_{N U}\left[1-\max \left(\frac{r_{b}}{r_{N L}}, \frac{\alpha_{G}}{\left(1-\alpha_{S}\right) \alpha_{N C}}\right)\right]\right),
$$

where $C_{N W}$ is a user-defined constant and $\mathrm{rMH}_{\mathrm{M}}$ given by Eq. (44).

If the bubble number densities, defined in Sec. III.B.1, are equal, the subdivision of the bubble surface is assumed to be fixed at $75 \%$ for the continuous liquid, taken from the convectible area, ${ }^{a} \mathrm{G} C L$, of the bubbles. Now

$$
a_{G O L}=\frac{n_{b, D L}}{3 n_{b, C L}+n_{b, D L}} w_{N U} a_{G C L}^{c}+\left(1-w_{N U}\right) a_{G O L}^{P}
$$

and

$$
a_{G C L}=\frac{3 n_{b, C L}}{3 n_{b, C L}+n_{b, D L}} w_{N U} a_{G C L}^{c}+\left(1-w_{N U}\right) a_{G C L}^{P},
$$




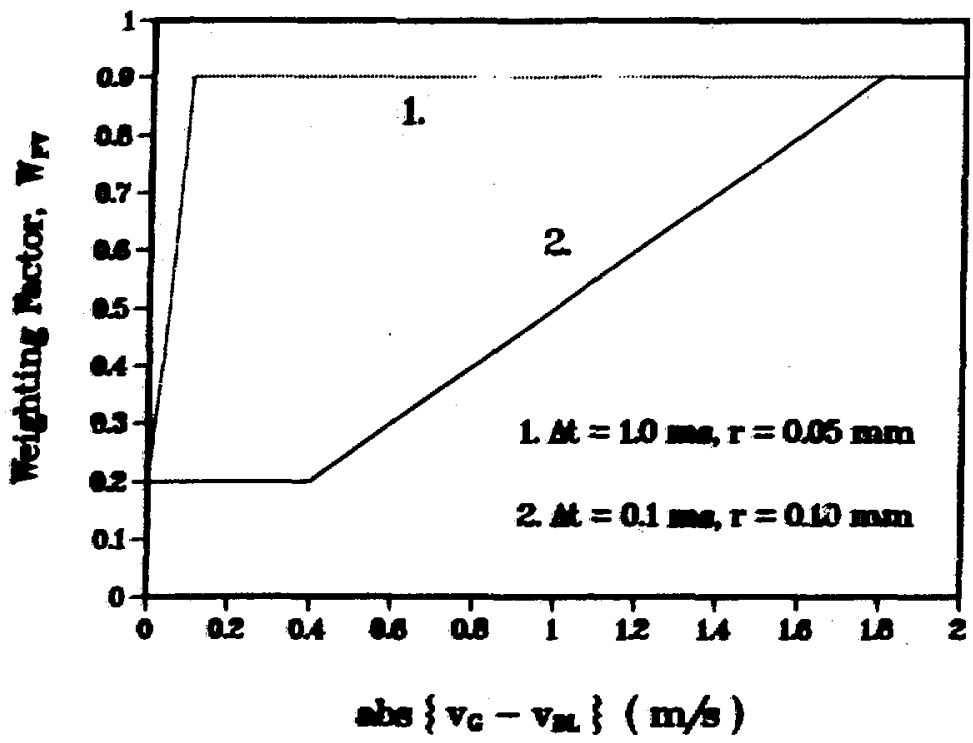

Fig. 18.

Weighting factor used for a combination of persisting and collision contact.

where $a_{G D L}^{p}$ is defined in the previous sections, and $a_{G C L}^{p}$ is the complementary value with $a_{G D}^{P}$ and $a_{G C L}^{P}$ adding up to the bubble convectible surface area, $a_{\text {GCL. }}^{c}$

G. Surface Area Multiplier

The user may want to increase the instantaneous interfacial areas independent of the models presented so far. A volume fraction dependent multiplier, $\xi$, is introduced. This multiplier is calculated for all cont inuous liquid coalescing regimes; for the continuous liquid nucleating regime it is set to unity.

$$
\xi=1+c_{A M} \min \left(\alpha_{C C}-\alpha_{B F R}, \max \left(0, \frac{\alpha_{G}}{1-\alpha_{S}}-\alpha_{B F R}\right)\right),
$$

where $\alpha_{B F R}$ and $\alpha_{C C}$ are the volume fraction limits for the churn-turbulent regine, as defined in Sec. II.D. The multiplier, is not used for the structure 
contacts. The value of the user-defined constant, $C_{A M}$, should not exceed 3. The multiplier is applied on the convectible surface areas of the discontinuous component under investigation.

\section{H. Interface Temperature}

For the surface tensions, the interface temperature, $T I$, is the main input argument. The surface tension is defined at the interface between the components, and the defirition of $T^{\prime}$ depends on whether both components are liquid, or one of them is vapor. The surface tensions are defined in Vol. VI, Sec. IV.

If one component is the vapor phase then

$$
T^{\prime}=T_{L m}
$$

where TLm is the bulk temperature of the liquid component $\mathrm{m}$.

If both components are liquid, then

$$
T^{\prime}=T_{E C}
$$

were $\mathrm{TEC}$ is the effective contact temperature, as given by Eq. (118) in Sec. IV.B. However, the contact partners are not L1 and P, but L1 and L2, or P and $L 2$, here $P$ are the solid particulates, L1 is liquid 1 , and L2 is liquid 2.

\section{K. Subdivisions}

The models of the previous sections are combined and applied for 10 of the 12 topologies. For topology 1 and 3 , no subdivision exists. Limiters have to be added at each step of the subdivision in order to prevent the sums of the subdivisions from exceeding the original convectible surface areas which are subdivided. The equations are listed in the order they are being programed at the end of subroutine INIIFA. The code sets all instantaneous interfacial areas wich, for a given topology, are not listed below, to zero. The order of the subscripts is irrelevant because 


$$
a_{k m}=a_{n k} .
$$

(158)

1. Topology 2 Subdivision. Sol id particulates are always discontinuous. The are totally immersed in liquid 1. The only other contact is with the structure if structure exists. If structure does not exist, the surface area, $a_{s}$, of the structure is zero. The total surface area of the solid particulates is always $3 \alpha_{p} / r_{p}$.

$$
\begin{aligned}
& a_{P S}=\frac{1}{2} \frac{\alpha_{p}}{1-\alpha_{S}} \min \left(\frac{3 \alpha_{p}}{r_{p}}, a_{S}\right), \\
& a_{P L 1}=\frac{3 \alpha_{p}}{r_{p}}-a_{P S},
\end{aligned}
$$

and

$$
{ }^{a_{S L 1}}=a_{S}-a_{P S} .
$$

2. Topology 4 and 7 Subdivision. For both topologies, liquid 1 is the cont inuous phase. Besides the solid particulates, the discontinuous phase, subscripts DP or D, is liquid 2 for topology 4 and vapor for topology 7 . There is no contact between the solid particulates and the discontinuous phase.

$$
\begin{aligned}
& a_{P S}=\frac{1}{2} \frac{\alpha_{p}}{1-\alpha_{S}} \min \left(\frac{3 \alpha_{p}}{r_{p}}, a_{S}\right), \\
& a_{D S}=\frac{1}{2} \frac{\alpha_{D P}}{1-a_{S}} \min \left(a_{D P 1}^{c}, a_{S}\right), \\
& a_{L 1 S}=a_{S}-a_{P S}-a_{D S}, \\
& a_{L 1 D P}=a_{D P L 1}^{c} \xi-a_{D S},
\end{aligned}
$$

64 
and

$$
a_{L T P}=\frac{3 \alpha_{p}}{r_{p}}-a_{P S} .
$$

3. Topology 5 and 6 Subdivision. For both topologies, liquid 1 is discontinuous and forms a common surface with the solid particulates. The subdivisions have to be valid even if either liquid 1 or particulate volume fraction is zero. The continuous phase, subscripts CP or C, is liquid 2 for topology 5 , and vapor for topology 6 . The liquid 1 droplet radius, $\mathrm{r}_{L} \mathrm{l}$, is

$$
r_{L 1}=\max \left(r_{\text {md }}, \frac{3 \alpha_{L 1}}{a_{L 1 C P}^{c}}\right),
$$

where $r_{\text {md }}$ is the user-defined minimum droplet radius. The comon radius of the liquid 1-particulate system is

$$
r_{\alpha}=\left(\alpha_{p}+\alpha_{L 1}\right)^{z_{1}}\left(\frac{\alpha_{p}}{r_{p}}+\frac{\alpha_{L 1}}{r_{L 1}}\right)^{-/ / 2} ;
$$

the common surface area is then

$$
a_{a r}=\frac{3\left(\alpha_{p}+\alpha_{L 1}\right)}{{ }^{r}}
$$

The liquid 1-solid particulate contact including that to the structure depends on the melting freezing indicator, iwf (see Fig. 14).

$$
C^{* *}=\min \left(1, \frac{\alpha_{L i}}{\left(\alpha_{p}+\alpha_{L 1}\right) \alpha_{L i m p}}\right)
$$




$$
\begin{aligned}
& \mathrm{C}^{++}=\min \left(1, \frac{\alpha_{p}}{\left(\alpha_{p}+\alpha_{L 1}\right) \alpha_{L M P}}\right) \text {. } \\
& C^{*}=1-\left(1-C_{P C P}\right) C^{\star \star} \text { for } I_{M F}=0 \text {, } \\
& C^{\star}=1-C_{\text {LoP }} C^{\star \star} \quad \text { for } I_{\text {MF }}=1 \text {, } \\
& C^{+}=1-C_{P C P} C^{++} \text {for } I_{M F}=0 \text {, } \\
& C^{+}=1-\left(1-C_{L C P}\right) C^{++} \text {for } I_{M F}=1 \text {, } \\
& a_{L 1 P, S}=\frac{1}{2} \frac{\alpha_{L 1}+\alpha_{P}}{1-\alpha_{S}} \min \left(a_{C,}, a_{S}\right) \text { for } i_{L P C}=0 \text {, } \\
& { }^{a_{P S}}=\mathrm{C}^{*} \mathrm{C}^{t+} a_{L 1 P, S} \quad \text { for } I_{L P C}=0 \text { or } I_{T}=5 \text {, } \\
& a_{L 1 S}=C^{+} C^{\star *} a_{L 1 P, S} \quad \text { for } I_{L P C}=0 \text { or } I_{T}=5 \text {, } \\
& a_{P S}=\frac{1}{2} \frac{a_{P}}{1-a_{S}} \min \left(\frac{3 a_{p}}{r_{P}}, a_{S}\right) \text { for } I_{L P C}=1 \text { and } I_{T}=6 \text {, } \\
& a_{L 1 S}=\frac{1}{2} \frac{a_{L 1}}{1-a_{S}} \min \left(a_{L 1 C P}^{c}, a_{S}\right) \text { for } L_{L P C}=1 \text { and } I_{T}=6 \text {, } \\
& a_{C S}=a_{S}-a_{L 1 S}-a_{P S} \\
& a_{L 1 P}=C_{L 1 P} C^{\star *} C^{++} a_{C M} \quad \text { for } I_{L P C}=0 \text { or } I_{T}=5 \text {, }
\end{aligned}
$$

and

66 


$$
\begin{aligned}
a_{L 1 P}= & \min \left[\frac{3 \alpha_{P}}{2 r_{P}}-\frac{a_{P S}}{2}+\frac{a_{L 1 C P}^{c}-a_{L 1 S}}{2},\right. \\
& \left.C_{P L 1} \frac{\alpha_{L 1} \alpha_{P}\left(r_{L 1}+r_{P}\right)\left(r_{L 1}^{2}+r_{P}^{2}\right)}{\left(1-\alpha_{S}\right) r_{L 1} r_{P} \max \left(r_{L 1}^{2}, r_{P}^{2}\right)}\right] \text { for } I_{L C P}=1 \text { and } I_{T}=6 \text {. }
\end{aligned}
$$

The above logic provides a choice for the contacts if vapor is the continuous phase, as described in Sec. IV.B. If ILPC $=1$, then the contact is based upon the collision model. Therefore, the sum of the subdivisions, listed in the following equations, has to reflect this choice as well. The multipliers $C^{*}$ $\mathrm{C}^{++}$and $\mathrm{C}^{+} \mathrm{C}^{\star *}$ force the instantaneous interfacial areas to zero if the particular component does not exist, as in Eq. (130):

$$
\begin{aligned}
& a_{L 1 P, C P}=a_{C N}-a_{L 1 P, S} \text { for } L_{L P C}=0, \\
& a_{P C P}=C^{*} C^{++} a_{L 1 P, C P} \text { for } I_{L P C}=0 \text { or } I_{T}=5, \\
& a_{L 1 C P}=C^{+} C^{\star \star} a_{L 1 P, C P} \text { for } I_{L P C}=0 \text { or } 1_{T}=5, \\
& a_{P C P}=\frac{3 a_{P}}{{ }_{P}}-a_{P S}-a_{L 1 P} \text { for } I_{L P C}=1 \text { and } I_{T}=6,
\end{aligned}
$$

and

$$
a_{L 1 C P}=a_{L 1 C P}^{c}-a_{L 1 S}-a_{L 1 P} \quad \text { for } L_{L P C}=1 \text { and } I_{T}=6 \text {. }
$$

For ILPC $=1$, liquid 1 droplets and particulates are supposed to have contact only through collision, and the common surface area, acu, is not used. For ILPC $=0$, the common surface area is calculated to have a reference area for the contact to the other components.

4. Topology 8 and 9 Subdivision. In both topologies, the flow field consists of only vapor and liquid 2. The discontinuous phase, subscripts oP or $D$, 
is liquid 2 for topology 8 , and vapor for topology 9 . The cont inuous phase, subscript $C P$, is vapor for topology 8 , and liquid 2 for topology 9.

$$
\begin{aligned}
& a_{D S}=\frac{1}{2} \frac{a_{D P}}{\alpha_{D P}+a_{C P}} \min \left(a_{G L 2}^{c}, a_{S}\right), \\
& a_{L 2 G .}=a_{G L 2}^{c} \xi-a_{D S} ;
\end{aligned}
$$

and

$$
{ }^{a} C S=a_{S}-a_{D S}
$$

5. Topology 10 Subdivision. Topology 10 is the cont inuous vapor topology with all components present. In addition to the contacts of topology 6, liquid 2 droplets are present. The droplet radii are

$$
r_{L 2}=\max \left(r_{\text {md }}, \frac{3 \alpha_{L 2}}{a_{G L 2}^{c}}\right)
$$

and

$$
r_{L 1}=\max \left(r_{m d}, \frac{3 \alpha_{L 1}}{a_{Q L 1}^{c}}\right),
$$

Where $r_{m d}$ is the user-defined minimum droplet radius.

The liquid 1-particulate common radius and area are

$$
r_{q_{1}}=\left(\alpha_{p}+\alpha_{L 1}\right)^{1 / 3}\left(\frac{\alpha_{p}}{r_{p}}+\frac{\alpha_{L 1}}{r_{L 1}}\right)^{-\frac{1}{3}}
$$

and 


$$
a_{C M}=\frac{3\left(\alpha_{p}+\alpha_{L 1}\right)}{{ }^{C M}} .
$$

The dimensionless functions for the liquid 1-particulate contact depend on the melting-freezing indicator, Imf (see Fig. 14).

$$
\begin{aligned}
& C^{\star *}=\min \left(1, \frac{\alpha_{L 1}}{\left(\alpha_{P}+\alpha_{L 1}\right) \alpha_{L M P}}\right), \\
& C^{++}=\min \left(1, \frac{\alpha_{p}}{\left(\alpha_{P}+\alpha_{L 1}\right) \alpha_{L M P}}\right), \\
& C^{*}=1-\left(1-C_{P C P}\right) C^{* *} \text { for } I_{M F}=0, \\
& C^{*}=1-C_{L C P} C^{* \star} \quad \text { for } I_{M F}=1, \\
& C^{+}=1-C_{P C P} C^{++} \text {for } I_{M F}=0,
\end{aligned}
$$

and

$$
C^{+}=1-\left(1-C_{P C P}\right) C^{++} \text {for } I_{M F}=1
$$

The contact to the structure is

$$
\begin{aligned}
& a_{L 1 P, S}=\frac{1}{2} \frac{\alpha_{P}+\alpha_{L 1}}{1-\alpha_{S}} \min \left(a_{C M}, a_{S}\right) \text { for } I_{L F C}=0, \\
& a_{P S}=C^{*} C^{++} a_{L 1 P, S} \text { for } I_{L P C}=0, \\
& a_{L 1 S}=C^{+} C^{* *} a_{L 1 P, S} \text { for } I_{L P C}=0,
\end{aligned}
$$

69 


$$
a_{P S}=\frac{1}{2} \frac{\alpha_{p}}{1-\alpha_{S}} \min \left(\frac{3 \alpha_{p}}{r_{p}}, a_{S}\right) \text { for } I_{L F C}=1 \text {, }
$$

and

$$
a_{L 1 S}=\frac{1}{2} \frac{\alpha_{L 1}}{1-\alpha_{S}} \min \left(a_{G L 1}^{c}, a_{S}\right) \quad \text { for } I_{L C P}=1 \text {. }
$$

For ILPC $=1$, I iquid 1 droplets and particulates are supposed to have contact only through collision, and the common surface area, acu, is not used. For ILPC $=0$, the common surface is calculated, and contact to the other components are calculated using this quantity.

The I iquid 2-structure contact is

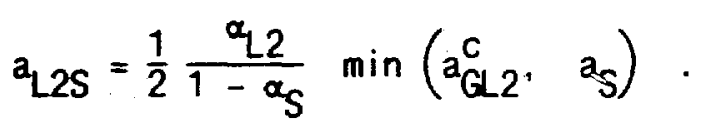

The continuous phase-structure contact is defined by the left-over surface area of the structure

$$
a_{G S}=a_{S}-a_{L 1 S}-a_{L 2 S}-a_{P S} .
$$

The contact of the three discontinuous partners of the two discontinuous velocity fields depends on wich value of ILPC has been given. For ILPC $=0$, the collision model is applied for two partners, one of which is the combined liquid 1-particulate field with aCM; for ILPC $=1$, there are three partners and separate three collision contacts.

$$
\begin{aligned}
a_{L 1 P, L 2}= & \min \left[\frac{a_{C M}-a_{L 1 P, S}}{2}, \frac{a_{L L 2}^{c}-a_{L 2 S}}{2},\right. \\
& \left.c_{P P} \frac{\left(\alpha_{P}+\alpha_{L 1}\right) \alpha_{L 2}}{1-\alpha_{S}} \frac{\left(r_{C M}+r_{L 2}\right)\left(r_{C M}^{2}+r_{L 2}^{2}\right)}{{ }_{C M} r_{L 2} \max \left(r_{C M}^{2}, r_{L 2}^{2}\right)}\right]
\end{aligned}
$$




$$
\begin{aligned}
& a_{L 1 P}=C_{L 1 P} C^{\star \star} C^{++} a_{C M} \text { for } L_{L P C}=0 \text {, } \\
& a_{L 1 L 2}=C^{+} C^{\star \star} a_{L 1 P, S} \text { for } I_{L P C}=0 \text {, } \\
& a_{P L 2}=C^{*} \cdot C^{++} a_{L 1 P, S} \text { for } L_{L P C}=0 \text {, } \\
& a_{L 1 P}=\min \left[\frac{3 \alpha_{p}}{2 r_{p}}-\frac{a_{P S}}{2}, \frac{a_{G L 1}^{c}-a_{L 1 S}}{2},\right. \\
& \left.C_{P L 1} \frac{\alpha_{L 1} \alpha_{P}}{1-\alpha_{S}} \frac{\left(r_{L 1}+r_{P}\right)\left(r_{L 1}^{2}+r_{P}^{2}\right)}{r_{L 1} r_{P} \max \left(r_{L 1}^{2}, r_{P}^{2}\right)}\right] \text { for } I_{L P C}=1 \text {, } \\
& a_{L 1 L 2}=\min \left[\frac{a_{G L 1}^{c}-a_{L 1 S}-a_{L 1 P}}{3}, \frac{a_{G L 2}^{c}-a_{L 2 S}}{3}\right. \text {, } \\
& \left.C_{P P} \frac{\alpha_{L 1} \alpha_{L 2}}{1-\alpha_{S}} \frac{\left(r_{L 1}+r_{L 2}\right)\left(r_{L 1}^{2}+r_{L 2}^{2}\right)}{r_{L 1} r_{L 2} \max \left(r_{L 1}^{2}, r_{L 2}^{2}\right)}\right] \text { for } I_{L P C}=1 \text {, }
\end{aligned}
$$

and

$$
\begin{aligned}
a_{P L 2}=\min & {\left[\frac{\alpha_{P}}{r_{P}}-\frac{a_{P S}+a_{L 1 P}}{3}, \frac{a_{G L 2}^{c}-a_{L 2 S}}{3},\right.} \\
& \left.c_{P P} \frac{\alpha_{P} \alpha_{L 2}}{1-\alpha_{S}} \frac{\left(r_{P}+r_{L 2}\right)\left(r_{P}^{2}+r_{L 2}^{2}\right)}{r_{P} r_{L 2} \max \left(r_{P}^{2}, r_{L 2}^{2}\right)}\right] \text { for } I_{L P C}=1 .
\end{aligned}
$$

Eventually, the sum of the subdivisions has to reflect the choice of ILPC, as mell. The multipliers $\mathrm{C}^{\star} \mathrm{C}^{++}$and $\mathrm{C}^{+} \mathrm{C}^{\star \star}$ force the instantaneous interfacial areas to zero if the particular component does not exist, as in Eq. (130):

$$
a_{L 2 G}=a_{G L 2}^{c}-a_{L 2 S}-a_{P L 2}-a_{L 1 L 2}
$$




$$
\begin{aligned}
& a_{L 1 P, G}=a_{C 1}-a_{L 1 P, L 2}-a_{L 1 P, S} \quad \text { for } I_{L P C}=0, \\
& a_{L 1 G}=c^{+} c^{\star *} a_{L 1 P, G} \quad \text { for } L_{L P C}=0, \\
& a_{P G}=c^{\star} c^{++} a_{L 1 P, G} \quad \text { for } L_{L P C}=0, \\
& a_{L 1 G}=a_{G L 1}^{c}-a_{L 1 L 2}-a_{L 1 P}-a_{L 1 S} \quad \text { for } I_{L P C}=1,
\end{aligned}
$$

and

$$
a_{P G}=\frac{3 \alpha_{P}}{r_{P}}-a_{P L 2}-a_{L 1 P}-a_{P S} \text { for } L_{L P C}=1 \text {. }
$$

6. Topology 11 Subdivision. Topology 11 is the liquid 1-cont inuous phase topology with all components present. The solid particulates are totally immersed in liquid 1. The only direct contact they can have is that to the structure and to the continuous phase. The droplet and bubble radii are

$$
r_{L 2}=\max \left(r_{m d}, \frac{3 a_{L 2}}{a_{L 1 L 2}^{c}}\right)
$$

and

$$
r_{G}=\max \left(r_{m b} \cdot \frac{3 \alpha_{G}}{a_{G L 1}^{c}}\right) .
$$

The nomenclature $r_{L 2}$ is used rather than $r_{d}$, and $r_{G}$ is used rather than $r_{b}$ because all subscripts are consistently representing the components. There can either be a collision, or a persisting contact, or both. The weighting factor is

$$
W_{P V}=\min \left(0.9, \max \left[C_{P V W}, \frac{\left|\vec{v}_{G}-\vec{v}_{L 2}\right| \Delta t C_{p V}}{2 \max \left({ }_{G}, r_{L 2}\right)}\right]\right) .
$$


The structure contacts are

$$
\begin{aligned}
& a_{P S}=\frac{1}{2} \frac{\alpha_{p}}{1-\alpha_{S}} \min \left(\frac{3 \alpha_{p}}{r_{p}}, a_{S}\right), \\
& a_{L 2 S}=\frac{1}{2} \frac{\alpha_{L 2}}{1-\alpha_{S}} \min \left(a_{L 1 L 2}^{c}, a_{S}\right), \\
& a_{G S}=\frac{1}{2} \frac{\alpha_{G}}{1-\alpha_{S}} \min \left(a_{G L 1}^{c}, a_{S}\right) .
\end{aligned}
$$

and

$$
a_{L 1 S}=a_{S}-a_{P S}-a_{L 2 S}-a_{G S} .
$$

For the persisting contact, based on the surface tensions, and limited by the ratio of the number densities, there are the following parameters:

$$
\begin{aligned}
& S_{A}=\sigma_{G L 1}-\sigma_{G L 2}-\sigma_{L 1 L 2}, \\
& S_{B}=\sigma_{G L 2}-\sigma_{G L 1}-\sigma_{L 1 L 2}, \\
& B=1-C_{C L G} \quad \text { for } S_{A} \geqq 0 \text { and } S_{B}<0, \\
& B=0.5 \text { for } S_{A}<0 \text { and } S_{B}<0,
\end{aligned}
$$

and

$$
B=C_{D L G} \text { for } S_{A}<0 \text { and } S_{B} \geqq 0 \text {. }
$$

The ratio of bubble and droplet number density is

73 


$$
\frac{n_{G}}{n_{L 2}}=\left(\frac{{ }_{L} 2}{r_{G}}\right)^{2} \frac{a_{Q L 1}^{c}}{a_{L 1 L 2}^{c}} .
$$

The persisting contact area, $a^{\sigma}$, needs an additional limiter if the multiplier, $\xi$, of Sec. IV.G is greater than unity. Otherwise the sums of the subdivision would be greater than the convertible surface area multiplied by $\xi$.

$$
\begin{aligned}
& a_{L 2 G}^{\sigma}=\min \left(B, 1-\frac{1}{3 \xi}\right) \min \left(1, \frac{n_{L 2}}{n_{G}}\right)\left(a_{G L 1}^{c}-a_{G S}\right) \text { for } r_{G}<r_{L 2} . \\
& a_{L 2 G}^{\sigma}=B\left(a_{G L 1}^{c}-a_{G S}\right) \min \left(1, \frac{n_{L 2}}{n_{G}}\right) \quad \text { for } r_{G} \geqq r_{L 2} \text { and } B>0.55 . \\
& a_{L 2 G}^{\sigma}=B\left(a_{L 1 L 2}^{c}-a_{L 2 S}\right) \min \left(1, \frac{n_{G}}{n_{L 2}}\right) \text { for } r_{G} \geqq r_{L 2} \text { and } B \leqq 0.55 .
\end{aligned}
$$

The collision contact area is

$$
\begin{aligned}
a_{L 2 G}^{v}=\min & {\left[\frac{a_{L 1 L 2}^{c}-a_{L 2 S}}{2}, \frac{a_{G L 1}^{c}-a_{G S}}{2},\right.} \\
& \left.c_{P P} \frac{\alpha_{G} \alpha_{L 2}}{1-\alpha_{S}} \frac{\left(r_{G}+r_{L 2}\right)\left(r_{G}^{2}+r_{L 2}^{2}\right)}{r_{G} r_{L 2} \max \left(r_{G}^{2}, r_{L 2}^{2}\right)}\right] .
\end{aligned}
$$

Combining collision and persisting contact,

$$
a_{L 2 G}^{P}=\xi\left[w_{P V} a_{L 2 G}^{V}+\left(1-w_{P V}\right) a_{L 2 G}^{\sigma}\right] \text {, }
$$

and allowing for the correction due to nucleation.

$$
W_{N U}=\max \left(0, C_{N U}\left[1-\max \left(\frac{r_{G}}{r_{N L}}, \frac{\alpha_{G}}{\left(1-\alpha_{S}\right) \alpha_{N C}}\right)\right]\right),
$$

74 


$$
a_{L 2 G}=\frac{w_{N U^{n}, L 2}}{3 n_{b, L 1}+n_{b, L 2}}\left(\xi a_{G L 1}^{c}-\alpha_{G S}\right)+\left(1-w_{N U}\right) a_{L 2 G}^{P},
$$

and

$$
a_{L 1 G}=\frac{w_{N U} 3 n_{b, L 1}}{3 n_{b, L 1}+n_{b, L 2}}\left(\xi a_{G L 1}^{c}-a_{G S}\right)+\left(1-w_{M U}\right)\left(\xi a_{G L 1}^{c}-a_{G S}-a_{L 2 G}^{P}\right),
$$

where the $n_{b}$ are the bubble number densities calculated in the nucleation model, Sec. III.B.1. The sum of the subdivision reflects the conditions of Eqs. (235) through (237). As mentioned in Sec. IV.D, there is a problem with the liquid-liquid surface area for configuration 1 of Fig. 15 and $\mathrm{rG}_{\mathrm{G}} \geqq \mathrm{rL2}$ (see also Fig. 17) because the change in liquid-liquid surface area is neglected. However, a ${ }_{\mathrm{L} 2 \mathrm{G}}$ has changed and can be substantially larger than the convectible surface area of liquid 2. Therefore, $a_{L 2 G}^{a}$ or its function must not be subtracted from a $a_{L 1 L 2}^{C}$. In this case, the collision contact area $a_{22 G}^{V}$ replaces $a_{L 2 G}^{P}$ in Eq. (243).

$$
\begin{gathered}
a_{L 1 L 2}=\xi a_{L 1 L 2}^{c}-a_{L 2 S}-\xi w_{P V} a_{L 2 G}^{V} \text { for } r_{G} \geq r_{L 2} \text { and } B>0.55 . \\
a_{L 1 L 2}=\xi a_{L 1 L 2}^{c}-a_{L 2 S}-a_{L 2 G}^{P} \text { for } r_{G} \geq r_{L 2} \text { and } B \leqq 0.55 \\
\text { or for } r_{G}<r_{L 2} .
\end{gathered}
$$

Finally, the particulate-structure contact reduces the liquici 1-particulate contact

$$
a_{L 1 P}=\frac{3 x_{P}}{r_{P}}-a_{P S} .
$$

7. Topology 12 Subdivision. Topology 12 is the liquid 2-continuous phase with all components present. Because liquid 1 and particulates are discontinuous, portions of the subdivision are similar to those of topology 5, 6, or 10 . 
The combination of melting-freezing criteria and corrections due to nucleation renders the subdivision rather complicated. The radii and the common liquid 1particulate surface area are

$$
\begin{aligned}
& r_{L 1}=\max \left(r_{m d} \cdot \frac{3 \alpha_{L 1}}{a_{L 1 L 2}^{c}}\right), \\
& r_{G}=\max \left(r_{m b} \cdot \frac{3 \alpha_{G}}{a_{G L 2}^{C}}\right), \\
& r_{C M}=\left(\alpha_{p}+\alpha_{L 1}\right)^{3 / 2}\left(\frac{\alpha_{p}}{r_{P}^{3}}+\frac{\alpha_{L 1}}{r_{L 1}}\right)^{-4 / 3},
\end{aligned}
$$

and

$$
{ }^{a} C_{1}=\frac{3\left(\alpha_{p}+\alpha_{L 1}\right)}{{ }^{r} c_{1}} .
$$

Again, $r_{G}$ is used rather than $r_{b}$, and $r_{L 1}$ instead of $r_{d}$. There can be either collision or persisting contact, or both. The weighting factor is

$$
W_{P V}=\min \left(0.9, \max \left[C_{P W M}, \frac{\left|\vec{v}_{G}-\vec{v}_{L 1}\right| \Delta t C_{P V}}{2 \max \left({ }_{G}, r_{C W}\right)}\right]\right) .
$$

To simplify the formulas, introduce a new variable, $\alpha[1$, only for the remainder of the topology 12 equations.

$$
\alpha_{L 1}^{R}=\frac{\alpha_{L 1}}{\alpha_{p}+\alpha_{L 1}}
$$


The contact of the liquid 1-particulate systems is calculated using the following functions:

$$
\begin{aligned}
& C^{\star \star}=\min \left(1, \frac{\alpha_{L 1}^{R}}{\alpha_{L M P}}\right), \\
& C^{++}=\min \left(1, \frac{1-\alpha_{L 1}^{R}}{\alpha_{L M P}}\right), \\
& C^{\star}=1-\left(1-C_{P C P}\right) C^{\star \star} \quad \text { for } I_{M F}=0, \\
& C^{\star}=1-C_{L C P} C^{\star \star} \quad \text { for } I_{M F}=1, \\
& C^{+}=1-C_{P C P} C^{++} \text {for } I_{M F}=0,
\end{aligned}
$$

and

$$
C^{+}=1-\left(1-C_{L C P}\right) C^{++} \quad \text { for } I_{M F}=1 .
$$

The contact between liquid 1 and the particulates is

$$
a_{L 1 P}=C_{L 1 P} c^{\star *} C^{++} a_{C H} .
$$

The contacts of the components to the structure are

$$
\begin{aligned}
& a_{L 1 P, S}=\frac{1}{2} \frac{\alpha_{P}+a_{L 1}}{1-\alpha_{S}} \cdot \min \left(a_{C M}, a_{S}\right), \\
& a_{P S}=C^{*} C^{++} a_{L 1 P, S},
\end{aligned}
$$

77 


$$
\begin{aligned}
& a_{L 1 S}=c^{+} c^{\star \star} a_{L 1 P, S}, \\
& a_{G S}=\frac{1}{2} \frac{a_{G}}{1-a_{S}} \min \left(a_{G L 2}^{c}, a_{S}\right),
\end{aligned}
$$

and

$$
a_{L 2 S}=a_{S}-a_{L 1 P, S}-a_{G S}
$$

For the persisting contact, based on the surface tensions, $S_{A}, S_{B}$, and $B$ have to be defined. Depending on the value of IMF, the surface tension of the liquid 1-particulate systems is that of the predominantly outside component.

$$
\begin{aligned}
& S_{A}=\sigma_{G L 2}-\sigma_{G L 1}-\sigma_{L 1 L 2} \text { for } I_{M F}=0 . \\
& S_{B}=\sigma_{G L 1}-\sigma_{G L 2}-\sigma_{L 1 L 2} \text { for } I_{M F}=0 . \\
& S_{A}=\sigma_{G L 2}-\sigma_{G P}-\sigma_{P L 2} \text { for } I_{M F}=1 . \\
& S_{B}=\sigma_{G P}-\alpha_{G L 2}-\sigma_{P L 2} \text { for } I_{M F}=1 .
\end{aligned}
$$

For the calculation of B, Eq. (145) specifies the exceptions

$$
\begin{array}{r}
B=\min \left(1-C_{C L G}, \max \left[0.5,1-C_{C L G}-0.5\left(1.15-\frac{a_{1.1}^{R}}{a_{L m P}}\right]\right)\right. \\
\text { for } S_{A} \geq 0 \text { and } S_{B}<0 .
\end{array}
$$

$$
B=0.5 \text { for } S_{A}<0 \text { and } S_{B}<0 .
$$

and

$$
B=C D L \text { for } S_{A}<0 \text { and } S_{B} \geq 0 \text {. }
$$


The ratio of the buble and liquid T-particulate number density is

$$
\frac{n_{G}}{n_{C H}}=\left(\frac{r_{M}}{r_{G}}\right)^{2} \frac{a_{G L 2}^{c}}{{ }^{a_{C W}}} .
$$

The persisting contact area, $a^{\sigma}$, nects an additional limiter if the multipliers, $\xi$, of Sec. IV.G. is greater than unity. Otherwise, the sums of the subdivision would be greater than the convectible surface area multiplied by $\xi$.

$$
\begin{aligned}
& a_{L T P, G}^{\alpha}=\min \left(B, 1-\frac{1}{3 \xi}\right) \min \left(1, \frac{n_{C M}}{n_{G}}\right)\left(a_{G L 2}^{c}-a_{G S}\right) \text { for } r_{G}<r_{C x} \text {. } \\
& a_{L T P, G}^{\sigma}=B\left(a_{G 2}^{c}-a_{G S}\right) \min \left(1, \frac{n_{C}}{n_{G}}\right) \text { for } r_{G}>r_{C A} \text { and } B>0.55 \text {. } \\
& a_{L P, G}^{\sigma}=B\left(a_{C}-a_{G S}\right) \min \left(1, \frac{n_{G}}{n_{C O}}\right) \quad \text { for } r_{G}>r_{C} \text { and } B \leq 0.55 \text {. }
\end{aligned}
$$

The collision contact is

$$
\begin{aligned}
& z_{L T P, G}^{V}=\min \left[\frac{a_{C M}-a_{L T P, S}}{2}, \frac{a_{G L}^{c}-a_{G S}}{2},\right. \\
& \left.c_{p P} \frac{\left(\alpha_{L 1}+\alpha_{p}\right)_{G}}{1-\alpha_{S}} \frac{\left(r_{G}+r_{G N}\right)\left(r_{G}^{2}+r_{G W}^{2}\right)}{r_{G} r_{C W} \max \left(r_{G}^{2}, r_{G}^{2}\right)}\right]
\end{aligned}
$$

Combining collision and persisting contact, we to distinguish wich of the liquid 1-particulate partners is predominantly outside (see Fig. 14).

$$
\sum_{L T P, G}^{P}=w_{P V} a_{L T P, G}^{V}+\left(1-w_{P V}\right) a_{L T P, G}^{\sigma} \quad \text { for } I_{W F}=1
$$




$$
a_{L I P, G}^{P}=\xi\left[w_{P V} a_{L 1 P, G}^{V}+\left(1-w_{P V}\right) a_{L 1 P, G}^{\sigma}\right] \quad \text { for }\left.\right|_{M F}=0 .
$$

For $I_{M F}=1$, the surface area multiplier is unity because particulates are predominantly forming the surface. They are rigid and their surface area is not influenced by the flow conditions. Allowing for the corrections due to nucleation leads : 0

$$
W_{N U}=\max \left(0, C_{N U}\left[1-\max \left(\frac{r_{G}}{r_{N L}}, \frac{\alpha_{G}}{\left(1-\alpha_{S}\right) \alpha_{N C}}\right)\right]\right) .
$$

As $\alpha_{\{1}^{R}$ decreases, the solid particulates become dominant. However, nucleation does not occur inside the particulates, so an additional limiter is applied, with $\alpha_{L}^{R}$ as the variable

$$
\begin{aligned}
a_{L, P, G}= & \frac{w_{N U} n_{b, L 1}}{n_{b, L 1}+3 n_{b, L 2}} \min \left(1,2 a_{L 1}^{R}\right)\left(\xi a_{G L 2}^{c}-a_{G S}\right) \\
& +\left(1-w_{N U}\right) a_{L 1 P, G}^{P} \\
a_{L 1 G}= & \frac{3 n_{b, L 2}+n_{b, L 1}\left[1-\min \left(1,2 a_{L 1}^{R}\right)\right]}{n_{b, L 1}+3 n_{b, L 2}} w_{W U}\left(\xi a_{G, 2}^{c}-a_{G S}\right) \\
& +\left(1-w_{N U}\right)\left(\xi a_{G L 2}^{c}-a_{G S}-a_{L 1 P, G}^{P}\right),
\end{aligned}
$$

where $n_{b}$ is the bubble number density calculated in the nucleation model, Sec. 111.B.1. The value of aL1P,G is subdivided betw liquid 1 and the particulates, forcing the values to zero if the component volume fraction is zero as

$$
a_{L 2 G}=C^{+} C^{* *} a_{L 1 P, G}
$$


and

$$
a_{P G}=C^{\star} c^{++} a_{L 1 P, G}
$$

The contact between the liquid 1-particulate system and the cont inuous liquid needs to be complementary to the contacts above, so all conditions are effective.

$$
\begin{aligned}
a_{L I P, L 2}=\xi a_{C M}-a_{L 1 P, S}-\xi w_{P V} a_{L 1 P, G}^{V} \text { for } I_{M F}=0 \\
\text { and } r_{G} \geq r_{C M} \text { and } B>0.55 .
\end{aligned}
$$

Instead of using Eqs. (279) and (280) to evaluate the last term of the Futs, only the collision contribution is subtracted to prevent negative instantaneous interfacial areas [see the description of Eq. (243)]. Additionally, the influence of nucleation on Eq. (283) through Eq. (286) is neglected to simplify the correlations.

$$
\begin{array}{ll}
a_{L 1 P, L 2}=a_{C 1}-a_{L 1 P, S}-w_{P V} a_{L I P, G}^{V} & \text { for } I_{W F}=1 \\
& \text { and } r_{G} \geqq r_{C M} \text { and } B>0.55 .
\end{array}
$$

In Eq. (284), the solid particulates are predominantly outside and the multiplier, $\xi$, is unity. The rest of the complementary conditions yields

$$
\begin{aligned}
& a_{L 1 P, L 2}=\xi a_{C M}-a_{L 1 P, S}-a_{L 1 P, G}^{P} \text { for } I_{M F}=0 \text { and } r_{G}<r_{L 2} \\
& \text { or for } I_{W F}=0 \text { and } r_{G} \geq r_{L 2} \text { and } B \leq 0.55 \\
& a_{L 1 P, L 2}=a_{C 1}-a_{L 1 P, S}-a_{L 1 P, G}^{P} \text { for } I_{M F}=1 \text { and }{ }_{{ }_{G}}<r_{L 2} \\
& \quad \text { or for } I_{M F}=1 \text { and } r_{G} \geq r_{L 2} \text { and } B \leq 0.55
\end{aligned}
$$


These two equations use $a^{P}$ for the same reason as in Eq. (244). Finally,

$$
a_{L 1 L 2}=C^{+} C^{* *} a_{L 1 P, L 2}
$$

and

$$
a_{P L 2}=C^{\star} C^{++} a_{L 1 P, L 2}
$$

\section{SHFACE ANA CONECTION}

In Step 4 of the AFOM algorithm, the convectible surface areas are convected with end-cf-time-step velocities. As mentioned in Sec. 1, there are two compiler options for two models of area convection. Figure 1 shows the two models represented by the two parallel dotted lines, ARCV1 for area convection model 1 and ARCV2 for area convection model 2 . Model 1 is the base model and has been used frequently, whereas model 2 is more experimental and its complicated logic may have limited validity. Sumarizing major features and differ-ences of the two models, model 1 has a convectible surface area for each velocity component, thereas in model 2 , the convectible surface areas are used only in conjunction with the discontinuous components. In model 1, the areas are convected with the velocities of the velocity component they are associated to, and that is done by defining an area per unit mass which is convected similarly to the energy. In model 2, the areas are convected with the velocity of the discontimuous velocity components, and several limitations and assumptions have to be introduced. In model 1 , the differencing sctieme is the same as for the other convection equation, so all options are available. In model 2, only donor-cell differencing can be applied. Both models are coded in subroutine AFDus4.

This section is divided so that area convection model 1 is described first, with all necessary transformations in all AFDM steps, and then model 2 is described.

\section{A. Area Convection Model 1}

In Step 4 of the AFDM algorithm, the surface area convection Eq. (52) is solved with the PHS set to zero. However, for model 1 , the convectible surface 
area has to be transformed to comply with the conditions of the model, wich is to be explained in this section. Eventually. Eq. (58) of Vol. I is obtained with

$$
\frac{\partial}{\partial t}\left(\bar{p}_{q} a_{q}^{p}\right)+\sum_{m \in q} \nabla \cdot\left(\bar{p}_{m} a_{q}^{p} \vec{v}_{q}\right)=0,
$$

where ap is the interfacial area per unit mass for momentum field $q$, and $q=1$ does nol include the solid particulates. Equation (289) is similar to the energy convection equation. The difference equation is given in Vol. I Eq. (59). The interfacial area per unit mass, ap, is a function of the associated surface area, $a^{a}$, with

$$
a_{q}^{p}=\sum_{m \in q} \frac{\alpha_{q}^{a}}{\bar{p}_{m}} \quad \text { for } q=1,2,3 \text { and } q \in m
$$

where momentum field $q=1$ consists of density component $m=L 1$, momentum field $q=2$ consists of density component $m=L 2$, and momentum field $q=3$ consists of density components $m=G 1, G 2$, G3. If $\bar{p}_{m}$ vanishes so does $a_{q}^{p}$.

$$
a_{q}^{p}=0 \quad \text { for } \bar{p}_{m}=0, \quad q \notin m .
$$

Therefore, each momentum field that exists in a given cell has to have an interfacial area associated to it. The convectible surface areas, ac, of Eq. (52) are defined between the discontinuous and the continuous phases. Table 1 lists the convectible surface areas for a given topology. The number of convectible surface areas is equal to the number of discontimous momentum fields. The missing surface area for the continuous momentum field has to be defined. Here, we use the simple approach that the associated surface area of the continuous momentum field is equal to the sum of all convectible surface areas. Table 111 lists all associated surface areas as functions of the convectible surface areas for the 12 topologies. The terms $I_{b}$ and $I_{C L}$ are defined 
in Eqs. (27) through (30). The first column of the associated surface areas represents the value of the continuous momentum field. The non-listed areas are zero. With Table 111, the two necessary transformations can be performed:

$$
a_{f}^{c}+a_{q}^{a}
$$

and

$$
a_{q}^{a}+a_{f}^{c}
$$

where the subscript $i$ denotes the interface between two momentum fields. For Eq. (293), the first column of the associated surface areas of Table 111 is not used because it represents the continuous phase which is determined only in Eq. (292). The two transformations are used in the initiation, in Step 1, and Step 4 of the AFDM algorithm, as show in Fig. 1. For model 1, ARCV1, the left path of the two parallel dotted vertical lines is taken. As the vaiues of af are determined in subroutine INICEL, the first transformation is done for all fields using Eq. (292). For all liquid single phase cells, i.e., topologies 2 through 5, Eq. (293) is used to obtain the values for $\mathrm{ac}_{\mathrm{f}}$ which are used in conjunction with Eq. (52) for the source terms. There are two transformations necessary during the determination of the continuous phase in two-phase cells. To start calculation of the levitation criterion, a maximum of two values for ${ }_{a} C_{f}$ have to be knom, as there are the convectible surface areas between all liquid momentum fields present and the vapor momentum field. Equation (293) for $f=G L 1$ and $f=G 2$ is used. The levitation criterion results in the evaluation of the new continuous phase. Consequently, the new values for $a c_{f}$ of the two-phase cells have to be obtained using Eq. (293) for all $f$. If the topologies 10,11 , or 12 change, a correction needs to be applied, wich is described in Sec. V.A.1. Finally, all new ${ }^{a} C_{f}$ for two-phase cells are known and are updated in the subsequent part of subroutine INIIFA. To perform the calculation of the convection transport of interfacial areas in Step 4 , Eq. (289) is used and all $\mathrm{ac}_{\mathrm{f}}$ have to be transformed to $\mathrm{a}^{\mathrm{a}}{ }_{\mathrm{q}}$ using Eq. (292). When the topclogy switches from topology 10,11, or 12 there can be a step-wise change in convectible surface area $\mathrm{ac}_{\mathrm{f}}$ when using Table 111 for 
TALE III

TRMEFOMMATIOWS EETWEEN COMECTIELE MD

associated IMTERFACIAL MMEAS

Convectible

Surface

Topology $1 \$$ ICL Areas Associated Surface Areas

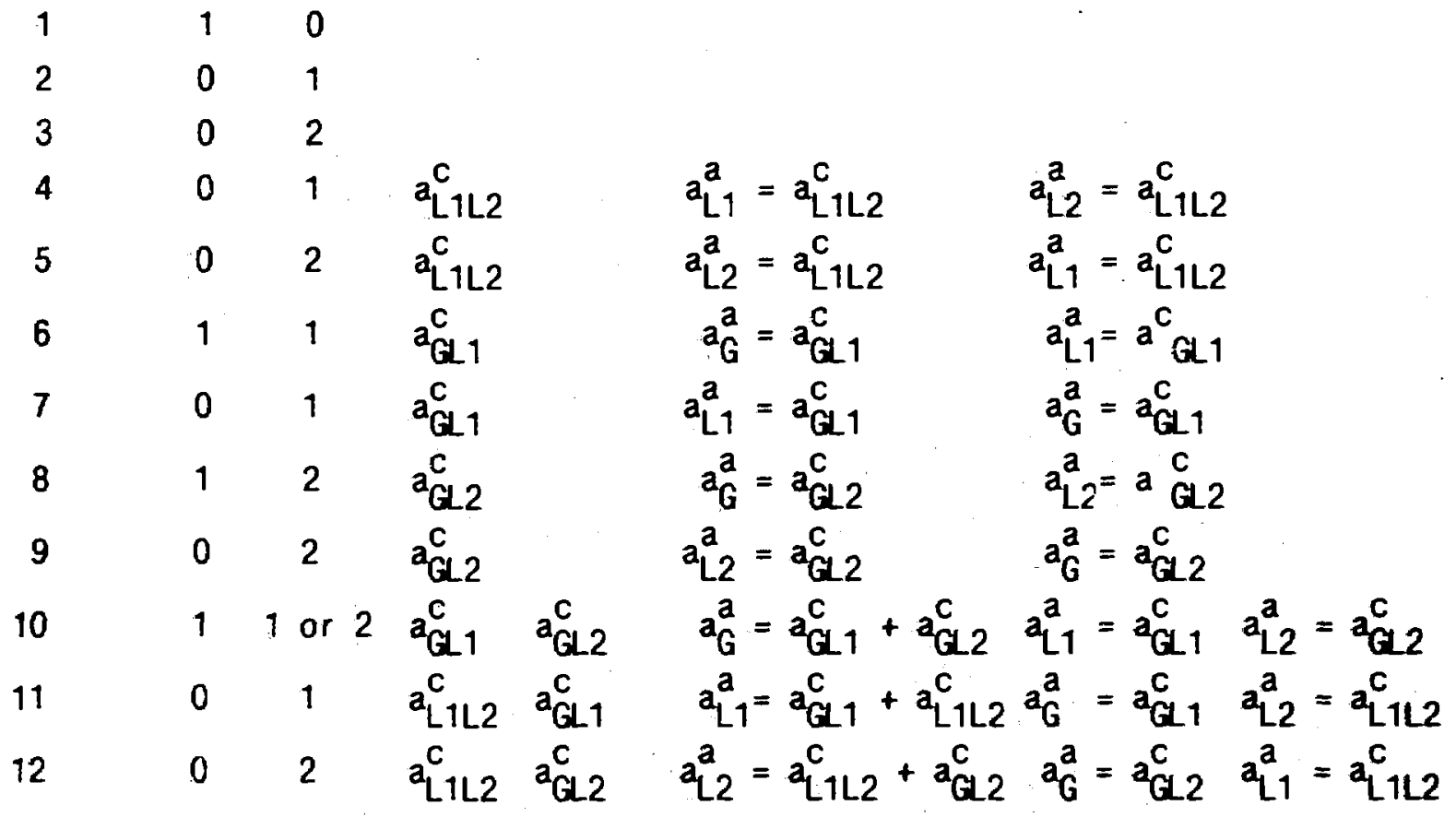

transformation. The assumption that the continuous momentum field has as associated surface area consisting of the sum of both convectible surface areas is irrelevant as long as for the same topology, the retransformation with Eq. (293) does not use this value. At the switch however, the value is used and a different transformation must be provided. According to Ref. 17, Table IV lists the convectible surface areas, $\mathrm{ac}_{\mathrm{f}}$, as functions of the associated surface areas, $\mathrm{a}^{\mathrm{a}} \mathrm{q}$, and the previous and current topologies, which establishes the transformation 


$$
\begin{aligned}
\text { for } I_{T}^{n+1}=10 \text { and } q=G \\
a_{q}^{a}+a_{f}^{c} \quad \text { for } I_{T}^{n+1}=11 \text { and } q=L 1 \\
\text { for } I_{T}^{n+1}=12 \text { and } q=L 2 .
\end{aligned}
$$

where $I_{T}^{n+1}$ is the topology of the current time step. If a dash is listed in Table IV, the convectible surface area is that from Table III, so no correction needs to be performed. Even with the correction, step-wise changes of convectible surface areas at the topology switch are inevitable. For example, if liquid 2 formed droplets in topology 10 of the previous time steps but consists of droplets in topology 11 of the current time step, the value of $\mathrm{aL}_{\mathrm{L} 1 \mathrm{~L} 2}$ is taken from $a_{G L 2}^{c}$ of the previous time step, irrespective of what the value of a\& 2 was before.

\section{B. Area Convection Model 2}

As for area convection model 1, the surface area convection Eq. (52) is solved with the RAS set to zero. However, only the convectible surface areas, $a^{c}$, as listed in Table 1 , are convected with the velocities of the discontinuous momentum fields. There is no need for a transformation, so

TALE IV

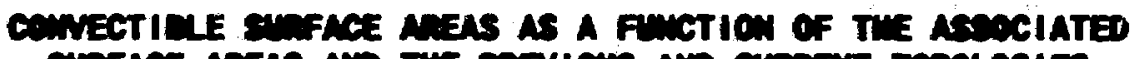

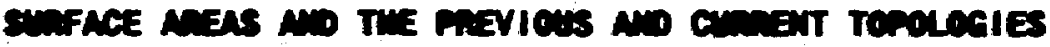

$\begin{array}{llllllllll}\text { Current Topology } & 10 & 10 & 10 & 11 & 11 & 11 & 12 & 12 & 12\end{array}$

\begin{tabular}{cccccccccc}
\hline Previous Topology & 10 & 11 & 12 & 10 & 11 & 12 & 10 & 11 & 12 \\
\hline$a_{L 1 L 2}^{c}$ & -- & -- & - & -- & - & $a_{L 1}^{a}$ & - & $a_{L 2}^{a}$ & - \\
$a_{G 1}^{c}$ & -- & $a_{G}^{a}$ & -- & $a_{L 1}^{a}$ & -- & - & -- & - & - \\
$a_{G 2}^{c}$ & -- & -- & $a_{G}^{a}$ & -- & -- & -- & $a_{L 2}^{a}$ & -- & -
\end{tabular}




$$
\frac{\partial a_{f}^{c}}{\partial t}+\nabla \cdot\left(a_{f}^{c} \vec{v}_{q}\right)=0
$$

where the subscript $f$ stands for L1L2, GL1, or $G L 2$, and $q$ is the subscript for the morrentum field. There are only as many momentum fields to account for as there are discontinuous components. To associate the subscript $f$ to $q$, references will be defined in Secs. V.B.1 and V.B.2. The problem with this approach arises when the difference equation has to be written for two neighboring cells with different topologies.

Four adjacent cells can contribute to a given cell with four different sets of discontinuous components. This renders any discretization other than a simple donor-cell differencing incompatible. Omitting the superscript $c$ in $\mathrm{a}^{\mathrm{C}}$ for reason of simplification, the difference equation is

$$
\begin{aligned}
& a_{f, i j}^{n,+1}=a_{f, i j}^{n_{1}}-\Delta t\left[\frac{\left\langle a_{f}^{n} \tilde{v}_{q}^{n+1}\right\rangle_{i, j+\hbar z}}{\Delta z_{j}}-\frac{\left\langle a_{f}^{n} \tilde{v}_{q}^{n+1}\right\rangle_{i, j-k_{z}}}{\Delta z_{j}}\right. \\
& \left.+\frac{\left\langle a_{f}^{n} r \tilde{u}_{q}^{n+1}\right\rangle_{i+k_{,}}}{r_{i}^{\Delta r_{i}}}-\frac{\left\langle z_{f}^{n} r \tilde{u}_{q}^{n+1}\right\rangle_{i-k_{,}, i}}{r_{i}^{\Delta r_{i}}}\right]
\end{aligned}
$$

Note that $f^{\prime}$ and $f$ may represent different subscripts. The first term inside the brackets is the convection term at the top of the cell under investigation; the second, third, and fourth are the terms at the bottom, right, and left, respectively. Depending on the direction of the velocities, the four terms can be "outflow" or "inflow" terms.

1. Outflow Terms. The outflow terms depend only on the convectible surface areas of the cell under investigation and the four velocity components on each side of the cell. To associate the subscript $f$ to subscript $q$ of Eq. (296). Table $V$ is constructed $w i$ th the current topology of the cell under investigation. However, the logic of the code is based on vector-merge functions. Therefore, dumy q-values are calculated for topology 1 , but are multiplied with zero convectible surface areas. All other listed q-values are used. 


\section{TABLE $Y$}

\section{SLACARIPT RELATIOHSHIPS FOR OUTFLOW TERHS \\ USIMG AREA COMNECTIOH MOLEL 2}

Topology

Velocity Subscript, $q$, as a Function of the Convectible Area Subscript, $f$

$$
f=L 1 L 2 \quad f=G L 1 . f=G L 2
$$

\begin{tabular}{rrrr}
\hline 1 & $\star$ & $*$ & $*$ \\
2 & & & \\
3 & $L 2$ & & \\
4 & $L 1$ & & \\
5 & & L1 & \\
6 & & $G$ & L2 \\
7 & & & G \\
8 & & & L2 \\
9 & & L1 & G
\end{tabular}

* Calculated in the code, but multiplied with a zero area.

Where there is a blank in Tatle $V$, the velocities are set to zero. Having determined the associated subscripts $f$ and $q$, the outflow terms are set to zero if the velocity component points into the cell.

$$
\begin{array}{ll}
\left\langle a_{f}^{n} v_{q}^{n+1}\right\rangle_{i, j+\hbar k}=0 & \text { for } v_{q, i, j+\psi_{2}}^{n+1} \leqq 0 \\
\left\langle a_{f}^{n} v_{q}^{n+1}\right\rangle_{i, j-z_{2}}=0 & \text { for } v_{q, i, j-y_{2}}^{n+1} \geqq 0 \\
\left\langle a_{f}^{n} \tilde{u}_{q}^{n+1}\right\rangle_{i+y_{, j}}=0 & \text { for } \tilde{u}_{q, i+\hbar, j}^{n+1} \leqq 0
\end{array}
$$




$$
\left\langle z_{f}^{n} \tilde{u}_{q}^{n+1}\right\rangle_{i-y_{2}, j}=0 \quad \text { for } \tilde{u}_{q, i-z_{2}, j}^{n+1} \geq 0 .
$$

Equation (296) is then used to update $\tilde{a}_{f}, i_{j}$ with the outflow terms inside the brackets, where $f=f^{\prime}$.

2. Inflow Terms. The inflow terms depend on the condition in both the cell under investigation and the neighboring cell where the flow comes from. If the topologies of the two neighboring cells are different, the contribution to the convectible surface area, $a_{f}{ }^{\prime}, i j$, of the cell under investigation can be from a neighboring convectible surface area with a different subscript, f. So, two different subscripts. $f^{\prime}$ and $f$, are associated. The velocity of the inflow surface area is always the velocity of the discontinuous momentum field. There are four basic principles. First, if both cells have the same discontinuous component, their convectible surface areas are associated. Second, if a discontinuous component switches to a continuous component and vice versa and if both components are present in the two neighboring cells, the convectible surface areas with the same subscript are associated. Third, if a discontinuous component is convected into a cell where this component did not exist yet, the convectible inflow area contributes to the convectible surface area betwen the new discontinuous component and the prevailing continuous phase. Fourth, if a cont inuous component is convected into a cell where it did not exist yet, it does not contribute to any convectible surface area.

Looking at each of the convectible surface areas in the cell under investigation, those contributions have to be accounted for that represent non-zero outflows from the neighboring cells. We therefore rewrite Table $V$ in terms of the outflow convectible area fluxes, ok, where

$$
o_{k}=z_{f}^{n} \tilde{v}_{q}^{n+1} \quad k=1,2,3
$$

where $v$ represents either the axial or radial velocity component, and with $k$ as the subscript for the combination out of three possible combinations. Table $V I$ lists the subscripts of the convectible surface area, $f$ of $a f$, and the subscript of the momentum field, $q$ of $\tilde{v}_{q}^{n+1}$, for the subscripts of the outflow 


\section{TABLE VI}

\section{SUBSCRIPT RELATIOUSHIPS AS A FUNCTION OF THE SUBSCRIPTS USED IN OUTFLOW FROM A MEICHBORIMG TOPOLOCY}

Neighboring

Topology
Subscripts of the Convectible Surface Area and Momentum Field $(f, q)$ for the Subscript $k$ of the Outflow Convectible Area Flux
$k=1$
$k=2$
$k=3$

$\begin{array}{rlll}1 & & & \\ 2 & & & \\ 3 & (L 1 L 2, L 2) & & \\ 4 & (L 1 L 2, L 1) & (G L 1, L 1) & \\ 5 & & (G L 1, G) & (G L 2, L 2) \\ 6 & & (G L 1, L 1) & (G L 2, G) \\ 7 & & (G L 1, G) & \\ 8 & & & (G L 2, G) \\ 10 & (L 1 L 2, L 2) & & \end{array}$

convectible area fluxes, $k$ of $o_{k}$, as a function of the neighboring topology. The values of ok are then used to determine the contributions due to inflow to the convectible surface areas. All information is listed in Table vil. The topologies are gathered in three groups: topologies $1,6,8$, and 10 into the group with vapor continuous flow, topologies $2,4,7$, and 11 into the group with liquid 1 continuous flow, and topologies 3, 5, 9, and 12 into the group with liquid 2 continuous flow. Depending on the groups of the donor and acceptor cells, the contributing outflow convectible area fluxes are lisied as they contribute to the convertible surface areas $a_{f}{ }^{\prime}, f^{\prime}=L 1 L 2, G L 1$, and GL2, of the acceptor cell. If a dash is listed in Table VIl, the contribution is zero. The contributions have to be evaluated for the top, bottom, left and right neighboring cells. Equation (296) is then used to update $\tilde{a}^{n}$ ', with all outflow contribution ok $\left(a_{f}^{n}, \tilde{v}_{q}^{n+1}\right)$. 


\section{TMEL VII}

INFLOW COWTRIEUTIONS USIMG AFDM NREA CONECTION MODEL 2

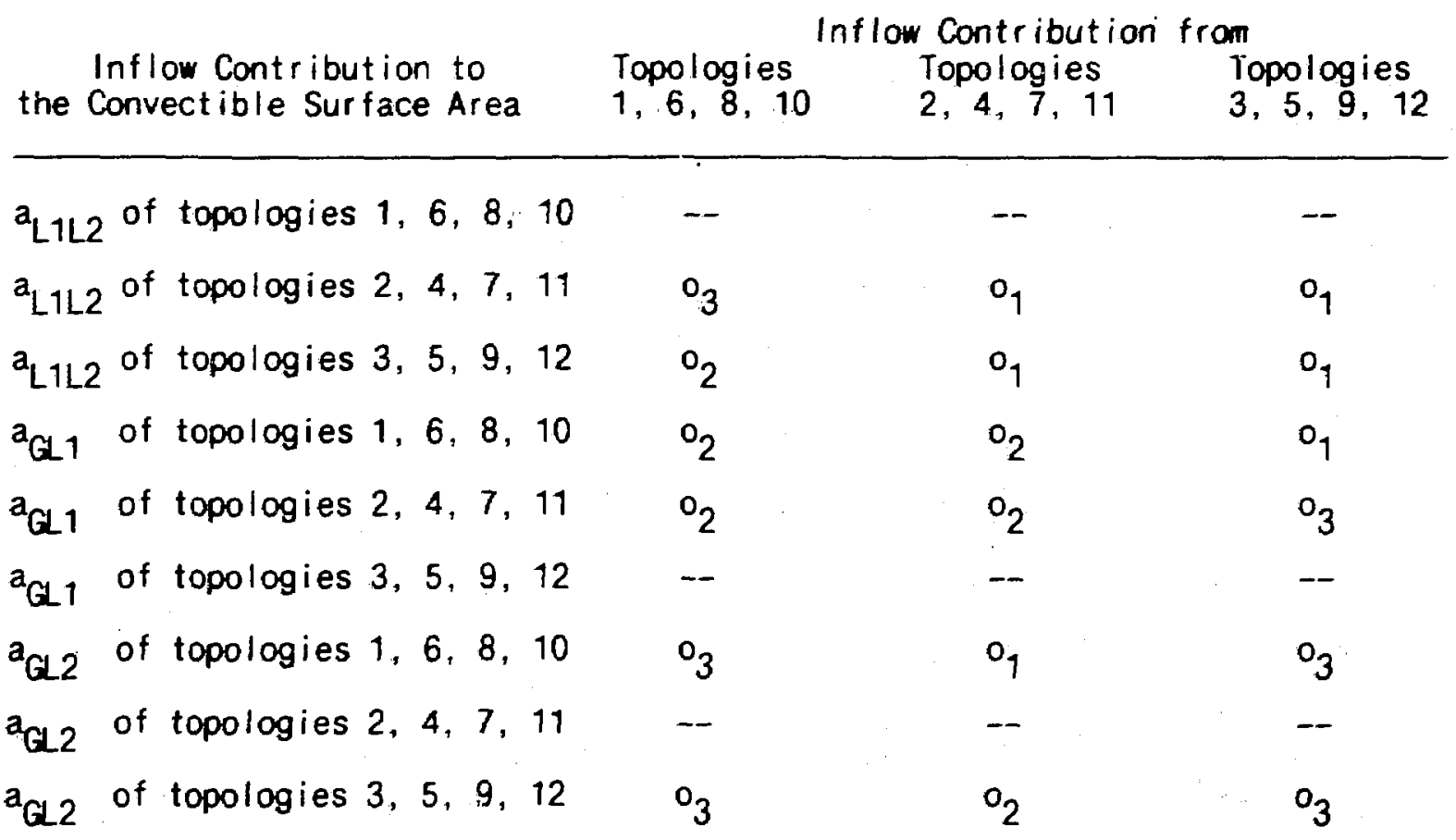

\section{IMITIAL CONDITIOUS AND USER IIMUT}

Before transient calculations can be started, the convectible surface ar eas need to be initiated. Figure 1 shows that the subrout ine INICEL is used to calculate either $a^{a}$ or $a^{C}$ for area convection model 1 or 2 , respectively. For most of the models of this volume, user-defined constants have been introduced to provide the user with a tool to change the values over the input file. Both features are described in this section.

\section{A. Initial Conditions}

The input for the convectible surface area is region wise. Two values contribute to the area calculation: the volume fraction and the ratius of the equivalent sphere. Equation (1) is used to calculate the convectible surface area, $a^{c}$. The radius of the equivalent sphere has to be provided for all momentum fields, even if it is anticipated that a momentum field becomes the continuous phase. For the momentum field of liquid 1 and solid particulates, only 
liquid 1 contributes to $a^{C}$. The solid particulates are given their own radius, ${ }^{\prime} p$. Which stays constant. In case that their interfacial surface area has to be accounted for, it is simply $a_{p}=3 \alpha_{p} / r_{p}$. As the input is region wise, all cells of a region are given the same values for $a^{C}$ and $r_{p}$.

For each region, subroutine INICEL calculates the topology using the volune fractions and the regime flag which may be provided in the input by the name REGFL. Therefore, the levitation criterion need not be applied. If the regime flag is zero, no levitation is anticipated, liquid is the continuous phase, and the phase indicator, $\left.\right|_{\phi}$, is zero, see Eq. (27). If the regime flag is unity, levitation is anticipated, vapor is the continuous phase, and $l_{\phi}$ is unity. The levitation criterion is valid only for vapor volume fractions aCC $<\alpha_{G} /\left(1-\alpha_{S}\right)<\alpha_{C D}[$ see Eqs. (23) and (24)]. In subroutine INICEL, these I iriits are taken care of by

$$
I_{\phi}=0 \quad \text { for } \frac{\alpha_{G}}{1-\alpha_{S}} \leqq \alpha_{C C}
$$

and

$$
I_{1}=1 \text { for } \frac{\alpha_{G}}{1-\alpha_{S}} \geq \alpha_{C D} \text {. }
$$

Therefore, Eqs. (292) and (293) overrule the regime-flag selection by the user.

\section{B. User Inout}

The user-defined constants for this volume are described in Vol. VII, Appendix E. With one exception, they are listed in Sec. IV of Appendix E, with the card identifier being wxC. The exception is the invariant gravitational acceleration, G, which is explained in conjunction with Eq. (44), and is given the value 9.8 in subroutine INICON. In the code, $G$ is represented by the name GRAVIT.

The following list refers the user-defined constants of this volume to the variable 10 of the code. It gives a short description of the constant, the present default value, and the equation number mere it is first referenced. 
It should be noted that most default values may not represent a balanced fluiddynamics approach. They may also differ over the whole range of fluid dynamics, as for example between the flow of cryogenic fluids and liquid reactor materials. Dimensionless quantities are usually less susceptive to changes than quantities with dimensions.

All user-defined constants listed hereafter can be changed through the input under the card identifier MXC.

Variable

\begin{tabular}{|c|c|c|c|c|}
\hline Constant & Equation & ID & Default & Description \\
\hline$\alpha C C$ & 20 & ALPCC & 0.50 & $\begin{array}{l}\text { The vapor-volume fraction at } \\
\text { the transition between churn } \\
\text { and cellular flow. }\end{array}$ \\
\hline$\propto C D$ & 19 & ALPCD & 0.65 & $\begin{array}{l}\text { The vapor-volume fraction at } \\
\text { the transition between droplet } \\
\text { and cellular flow. }\end{array}$ \\
\hline वLMP & 124 & ALPLMP & 0.01 & $\begin{array}{l}\text { The volume fraction of liquid } 1 \\
\text { or particles at which CPCP or } \\
\text { CLCP start to decrease linear- } \\
\text { ly to zero. }\end{array}$ \\
\hline aNC & 45 & ALPNC & 0.1 & $\begin{array}{l}\text { The maximum vapor-volume frac- } \\
t \text { tion for the noncoalescing bub- } \\
\text { bly regime. }\end{array}$ \\
\hline aNF & 31 & ALPNF & 0.95 & The no-flow volume fraction. \\
\hline$\alpha \mathrm{PF}$ & 30 & ALPPF & 0.7 & $\begin{array}{l}\text { The volume fraction multiplier } \\
\text { defining the influence of } \alpha_{p} \\
\text { on the cont inuous liquid defi- } \\
\text { nition. }\end{array}$ \\
\hline
\end{tabular}




\begin{tabular}{|c|c|c|c|c|}
\hline $\mathrm{C}_{\mathrm{AM}}$ & 152 & CAM & 0.0 & $\begin{array}{l}\text { The multiplier of the linear } \\
\text { variation of the surface area } \\
\text { multiplier, not to exceed } 3 \text {. }\end{array}$ \\
\hline $\mathrm{C}_{\mathrm{BC}}$ & 46 & $\mathrm{CBC}$ & 0.2 & $\begin{array}{l}\text { The multiplier of the particle } \\
\text { volume fraction to assess the } \\
\text { maximum vapor-volume fraction } \\
\text { for bubbly flow. }\end{array}$ \\
\hline $\mathrm{C}_{\mathrm{CLG}}$ & 142 & CCLG & 0.2 & $\begin{array}{l}\text { The minimum fraction of the } \\
\text { contact area between contin- } \\
\text { uous liquid and vapor. }\end{array}$ \\
\hline $\mathrm{C}_{\mathrm{COB}}$ & 105 & $\mathrm{CCOB}$ & 2.0 & $\begin{array}{l}\text { The multiplier for the coales- } \\
\text { cence rate of bubbles. }\end{array}$ \\
\hline $\mathrm{C}_{\mathrm{COD}}$ & 105 & $\mathrm{CCOD}$ & 0.01 & $\begin{array}{l}\text { The multiplier for the coales- } \\
\text { cence rate of droplets. }\end{array}$ \\
\hline CDLG & 142 & CDLG & 0.2 & $\begin{array}{l}\text { The minimum fraction of the } \\
\text { contact area between discon- } \\
\text { tinuous liquid and vapor. }\end{array}$ \\
\hline$C_{F D}$ & 81 & CFD & 0.5 & $\begin{array}{l}\text { The multiplier of the equilib- } \\
\text { rium radius reached by frag- } \\
\text { mentation of droplets by dynam- } \\
\text { ic forces. }\end{array}$ \\
\hline$C_{F L}$ & 101 & CFL & 1.0 & $\begin{array}{l}\text { The multiplier of the time con- } \\
\text { stant of the droplet fragment- } \\
\text { ation by flashing. }\end{array}$ \\
\hline$C_{F S}$ & 82 & CFS & 1.0 & $\begin{array}{l}\text { The multiplier of the time con- } \\
\text { stant of fragmentation of drop- } \\
\text { lets by dynamic forces. }\end{array}$ \\
\hline
\end{tabular}




\begin{tabular}{|c|c|c|c|c|}
\hline$C_{F T}$ & 77 & CFT & 1.0 & $\begin{array}{l}\text { The multiplier of the time con- } \\
\text { stant for fragmentation of bub- } \\
\text { bles by dynamic forces. }\end{array}$ \\
\hline$C_{F V}$ & 74 & CFV & 1.0 & $\begin{array}{l}\text { The multiplier of the viscos- } \\
\text { ity term for fragmentation by } \\
\text { dynamic forces. }\end{array}$ \\
\hline$c_{\mathrm{L} 1 \mathrm{P}}$ & 128 & CL1P & 0.5 & $\begin{array}{l}\text { The fraction of the common sur- } \\
\text { face of the liquid 1-particle } \\
\text { system at ILPC = } 0 \text { which repre- } \\
\text { sents the surface area between } \\
\text { liquid } 1 \text { and particles. }\end{array}$ \\
\hline$c_{\mathrm{LCP}}$ & 126 & CLCP & 0.2 & $\begin{array}{l}\text { The fraction of the liquid } 1 \\
\text { droplet surface that always } \\
\text { remains without a solid crust. }\end{array}$ \\
\hline$C_{M T}$ & 123 & CMT & 1.0 & $\begin{array}{l}\text { The multiplier of the liquid } 1 \\
\text { melting temperature to define } \\
\text { the melting/freezing threshold. }\end{array}$ \\
\hline$c_{N K C}$ & 70 & CNC & 10.0 & $\begin{array}{l}\text { The factor to define the gas } \\
\text { volume fraction at which the } \\
\text { nucleation model fades out. }\end{array}$ \\
\hline$c_{\mathrm{NL}}$ & 44 & CNL & 0.1 & $\begin{array}{l}\text { The multiplier of the square } \\
\text { of the threshold bubble radius } \\
\text { for the noncoalescing bubbly } \\
\text { regime. }\end{array}$ \\
\hline$c_{N U}$ & 152 & CNU & 0.9 & $\begin{array}{l}\text { The maximum value of the weight } \\
\text { factor defining the influence } \\
\text { of nucleation on the bubble } \\
\text { surface area. }\end{array}$ \\
\hline
\end{tabular}


CPCP

126

CPCP

0.2

The fraction of a particle surface area that always remains without a liquid $1 \mathrm{film}$.

$\mathrm{CPL1}_{1}$

128

CPL1

0.3

The multiplier for the effective contact area between liquid 1 and particles at ILPC = 1 , i.e., random collision.

$\mathrm{C}_{\mathrm{PM}}$

107

CPM

5.0

The ratio of the particle and liquid 1 volume fraction at which the droplet size source term becomes zero.

CPP

138

CPP

0.3

The multiplier of the effective contact area between discont inuous components at random collision.

CPV

150

CPV

1.0

The multiplier of the linear dependence of the weight factor for the combination of persisting and collision contact.

CPVM 150

CPVM

0.2

The lower limit of the weight factor for the combination of persisting and collision contact between discontinuous components.

$C_{R G}$

92

CRG

1.0

The multiplier of the equilibrium surface area reached by turbulence fragmentation. 


\begin{tabular}{|c|c|c|c|c|}
\hline $\mathrm{C}_{\mathrm{RTB}}$ & 105 & CRTB & 8.0 & $\begin{array}{l}\text { The multiplier of the exponent } \\
\text { of the probability for the coa- } \\
\text { lescence of bubbles. }\end{array}$ \\
\hline CRTD $_{\text {RT }}$ & 105 & CRTD & 1.0 & $\begin{array}{l}\text { The multiplier of the exponent } \\
\text { of the probability for the coa- } \\
\text { lescence of droplets. }\end{array}$ \\
\hline$C_{\theta}$ & 68 & CTHETA & $1.0^{+5}$ & $\begin{array}{l}\text { The multiplier of the exponent } \\
\text { to assess the number of nucle- } \\
\text { ating bubbles. }\end{array}$ \\
\hline $\mathrm{C}_{\mathrm{TT}}$ & 96 & CTT & 1.0 & $\begin{array}{l}\text { The multiplier of the time con- } \\
\text { stant for the fragmentation by } \\
\text { turbulence. }\end{array}$ \\
\hline$\triangle \mathrm{PHY}$ & 25 & DROHY & 100.0 & $\begin{array}{l}\text { The density difference to de- } \\
\text { fine the limits of the linear- } \\
\text { ized hysteresis at the } \\
\text { cellular-droplet regime's } \\
\text { transition }\left(\mathrm{kg} / \mathrm{m}^{3}\right) \text {. }\end{array}$ \\
\hline$\Delta p_{\min }$ & 44 & DROMIN & 10.0 & $\begin{array}{l}\text { The minimum density difference } \\
\text { between cont inuous and discon- } \\
\text { tinuous components }\left(\mathrm{kg} / \mathrm{m}^{3}\right) \text {. }\end{array}$ \\
\hline$\Delta V_{R T}$ & 68 & DVRT & 0.2 & $\begin{array}{l}\text { The reference velocity differ- } \\
\text { ence for the fragmentation of } \\
\text { bubbles by dynamic forces } \\
(\mathrm{m} / \mathrm{s}) \text {. }\end{array}$ \\
\hline 'LPC & 128 & ILPC & 0 & $\begin{array}{l}\text { The indicator for whether the } \\
\text { liquid 1-particle contact is } \\
\text { based on a collision model, }\end{array}$ \\
\hline
\end{tabular}


$I_{L P C}=1$, or on persisting contact, ILPC $=0$. for cont inuous vapor cells.

$\begin{array}{llll}\text { nDROP } 112 & \text { NDROP } & 1.0^{+7} & \begin{array}{l}\text { The number of newly created } \\ \text { droplets at condensation incep- } \\ \text { tion per fluid volume }\left(\mathrm{m}^{-3}\right)\end{array} \\ \mathrm{n}_{\max } 68 & \max & 1.0^{+11} & \begin{array}{l}\text { The maximum number of nucle- } \\ \text { at ing bubbles per fluid volume } \\ \left(\mathrm{m}^{-3}\right) .\end{array}\end{array}$

\begin{tabular}{|c|c|c|c|c|}
\hline$n_{\min }$ & 68 & NMIN & $1.0+5$ & $\begin{array}{l}\text { The minimum number of nucle- } \\
\text { ating bubbles per fluid volume } \\
\left(\mathrm{m}^{-3}\right) \text {. }\end{array}$ \\
\hline
\end{tabular}

$r_{m b} \quad 64$

RMB $\quad 1.0^{-6} \quad$ The minimum vapor bubble radius $(\mathrm{m})$.

\begin{tabular}{|c|c|c|c|c|}
\hline$r_{\mathrm{md}}$ & 63 & RMD & $1.0^{-6}$ & The minimum droplet radius (m) \\
\hline$r_{x b}$ & 64 & RXB & 0.1 & The maximum bubble radius $(\mathrm{m})$. \\
\hline$r_{x d}$ & 63 & RXD & 0.1 & The maximum droplet radius (m) \\
\hline$\theta_{0}$ & 68 & THETAO & $2.0^{-3}$ & $\begin{array}{l}\text { Threshold value for the non- } \\
\text { dimensional temperature differ } \\
\text { ence that defines the depar- } \\
\text { ture from saturation. }\end{array}$ \\
\hline ¿NuC & 70 & TCNUC & $1.0^{-4}$ & $\begin{array}{l}\text { The time constant for the nu- } \\
\text { cleation source term of the } \\
\text { convectible bubble surface } \\
\text { area }(s) \text {. }\end{array}$ \\
\hline
\end{tabular}




\begin{tabular}{|c|c|c|c|c|}
\hline$w_{\text {crit }}$ & 14 & WECR IT & 22.0 & $\begin{array}{l}\text { The critical Weber number at } \\
\text { the onset of droplet flow. }\end{array}$ \\
\hline WefB & 73 & WEFB & 10.0 & $\begin{array}{l}\text { The critical Weber number for } \\
\text { the fragmentation of bubbles. }\end{array}$ \\
\hline WeFD & 80 & WEFD & 13.0 & $\begin{array}{l}\text { The critical Weber number for } \\
\text { the fragmentation of droplets. }\end{array}$ \\
\hline
\end{tabular}

\section{MOMENCLATURE}

In this section, the nomenclature as given as a summary for the AFDM program in Vol. I is listed to facilitate the reader. In this volume we use only a part of the nomenclature. In addition, very limited use is made of local variables in the text that are not listed in the nomenclature.

\section{Variable Definition}

a Interfacial surface area per unit volume $\left[\mathrm{m}^{2} / \mathrm{m}^{3}\right]$

ap Convectible interfacial surface area per unit mass $\left[\mathrm{m}^{2} / \mathrm{kg}\right]$

a Viscous component of the interfield momentum-exchange coefficient $\left[\mathrm{N}-\mathrm{s} / \mathrm{m}^{4}\right]$

a Liquid/vapor velocity ratio [dimensionless]

[a] Three-by-three matrix representing the coefficients used to update the velocities based on mass-transfer results $\left[\mathrm{kg} / \mathrm{m}^{3}-\mathrm{s}\right]$

$a_{r} \quad$ Residual surface area in three-phase contact $\left[\mathrm{m}^{2} / \mathrm{m}^{3}\right]$ 
A Homogeneous condensation coefficient [w/m $\left.\mathrm{m}^{3}-\mathrm{K}^{2}\right]$

A Area, cell radial area $\left[\mathrm{m}^{2}\right]$

A Matrix of Jacobian derivatives used in the pressure iteration [variable dimensions]

$\vec{b} \quad$ Right-side vector in the equations to update the velocities based on mass transfer $\left[\mathrm{kg} / \mathrm{m}^{2}-\mathrm{s}^{2}\right]$

b Turbulent component of the interfield momentum-exchange coefficient $\left[N-s^{2} / m^{5}\right]$

B Fraction [dimensionless]

[B] Five-by-five Jacobian matrix used in the "two-phase" heat-and mass-transfer algorithm [variable dimensions]

[BS] Five-by-five Jacobian matrix used in the "single-phase" heat- and mass-transfer algorithm [variable dimensinns]

$\overrightarrow{\mathbf{B}}_{1}, \overrightarrow{\mathbf{B}}_{2}$, Vectors containing the pressure derivatives of the four $\vec{B}_{3}$, and $\vec{B}_{4}$ conservation relationships used Step 3 of the AFDM algorithm [variabie dimensions]

C Fraction [dimensionless]

C User-defined nondimensional or dimensional constant

$C^{\prime} \quad$ User-defined nondimensional or dimensional constant

$\vec{c} \quad$ Vector column representing the right sides of the equations used in the "two-phase" heat- and mass-transfer algorithm [variable dimensions ] 
$\overrightarrow{\mathbf{c}} \quad$ Right side of the relationship to maintain constant liquid/ particle internal energies in Step $3[\mathrm{~J} / \mathrm{kg}]$

$\mathrm{c}_{\mathrm{A}} \quad$ Total molar density $\left[\mathrm{kmol} / \mathrm{m}^{3}\right]$

$C_{D} \quad$ Drag coefficient [dimensionless]

$C_{G}$ Flow-regime-dependent part of the virtual mass coefficient [dimensionless ]

$c_{k} \quad$ Molar density of vapor species $k\left[\mathrm{kmol} / \mathrm{m}^{3}\right]$

$c_{m} \quad$ Velocity of sound for density component $m[\mathrm{~m} / \mathrm{s}]$

$c_{p} \quad$ Specific heat at constant pressure (heat capacity) [J/kg-K]

$\vec{c}_{S}$ Vector column representing the right sides of the equations used in the "single-phase" heat- and mass-transfer algorithm [variable dimensions]

$c_{t}$ Input fraction used for the Courant time-step control [dimensionless ]

$\mathrm{CT}_{\mathrm{r}}$ Convective term for the internal energy equation $\left[\mathrm{J} / \mathrm{m}^{3}\right]$

$\mathrm{CV}_{\mathrm{q}}$ Convective term for the momentum equation $\left[\mathrm{N} / \mathrm{m}^{3}\right]$

$c_{v}$ or $c$ Specific heat at constant volume (heat capacity) $[\mathrm{J} / \mathrm{kg}-\mathrm{K}]$ ( $\mathrm{c}_{v}$ preferred, unless used with other subscripts)

d Diameter [m]

D Error in one of the four conservation equations to be eliminated during the pressure iteration [variable dimensions] 

D Diffusion coefficient $\left[\mathrm{m}^{2} / \mathrm{s}\right]$
D Effective energy of vaporization/condensation [ $\mathrm{J} / \mathrm{kg}]$
$\vec{D} \quad$ Right-side vector used in solving the momentum equations $\left[\mathrm{kg} / \mathrm{m}^{2}-\mathrm{s}\right]$

$D_{h}$ or dh Hydraulic diameter [m] ( $D_{h}$ preferred)

e Specific internal energy $[\mathrm{J} / \mathrm{kg}]$

E A factor in the derivative of the homogeneous condensation term [K]

E The results of an energy sumation [J]

ef Internal energy change in fusion [ $/ \mathrm{kg}]$

elg Energy of vaporization $[\mathrm{J} / \mathrm{kg}]$

f Frequency $\left[s^{-1}\right]$

$f \quad$ Friction factor [dimensionless]

F View factor (Shape factor) [dimensionless]

F Viscosity function [dimensionless]

Fv Virtual mass force per unit volume $\left[\mathrm{N} / \mathrm{m}^{3}\right]$

$F_{12}$ View factor between liquid 1 and liquid 2 [dimensionless]

$\overrightarrow{\mathbf{g}} \quad$ Acceleration of gravity $\left[\mathrm{m} / \mathrm{s}^{2}\right]$

G General dependent variable function [arbitrary dimensions]

G Invariant acceleration $\left[+9.80 \mathrm{~m} / \mathrm{s}^{2}\right]$ 
Gr Grashof number $\left[8 r_{d}^{3} \rho_{G}\left(\rho_{L}-\rho_{G}\right) G / \mu_{G}^{2}\right.$ ] [dimensionless]

h Heat-transfer coefficient $\left[w / m^{2}-k\right]$

$h^{\prime} \quad$ Heat-transfer coefficient modified $t s$ treat $t$ ransfers between bulk vapor and another energy component $\left[\mathrm{W} / \mathrm{m}^{2}-\mathrm{K}\right]$

$h^{+} \quad$ Heat-transfer coefficient corrected for mass transfer $\left[\omega / m^{2}-K\right]$

For the following heat-transfer coefficients, usage without the commas is preferred.

$h_{G L m}$ or Heat-transfer coefficient between vapor and the liquid $m$ /vapor $h_{G, L m}$ interface $\left[W / m^{2}-K\right]$

$h_{G P}$ or Vapor-side heat-transfer coefficient for the particle-vapor $h_{G, P} \quad$ interface $\left[W / m^{2}-K\right]$

hGS or Vapor-side heat-transfer coefficient for the structure-vapor. $h_{G, S}$ interface $\left[w / m^{2}-K\right]$

$h_{G P}^{\prime} \quad$ Net heat-transfer coefficient between the vapor and the particles $\left[W / m^{2}-k\right]$

$h_{G S}^{\prime} \quad$ Net heat-transfer coefficient between the vapor and the structure $\left[W / m^{2}-K\right]$

$h_{\text {LmG }}$ or Heat-transfer coefficient between liquid $m$ and the liquid $m /$ vapor $h_{L m, G}$ interface $\left[w / m^{2}-K\right]$

$h_{P G}$ or Particle-side heat-transfer coefficient for the particle-vapor $h_{P, G}$ interface $\left[w / m^{2}-K\right]$ 
hSG or Structure-side heat-transfer coefficient for the structure-vapor hS, interface $\left[\mathrm{W} / \mathrm{m}^{2}-K\right]$

$h_{\ell g} \quad$ Heat of vaporization $[\mathrm{J} / \mathrm{kg}]$

H Heaviside function [dimensionless]

H Product of the interfacial area and the heat-transfer coefficient. Used with two energy component subscripts [W/m $\left.\mathrm{m}^{3}-K\right]$

$\bar{H}_{i} \quad$ Derivatives of the vapor energy equation with the vapor mole frac$t$ ion differentiated with respect to density $[\mathrm{W} / \mathrm{kg}-\mathrm{K}]$

$\tilde{H}_{i}^{(m)} \quad$ Component of the $[B]$ matrix containing terms of liquid energy equation $m$ differentiated with respect to independent variable $x_{i}$ [variable dimensions]

$\mathrm{H}_{\mathrm{i}}^{*} \quad$ Collection of derivatives of the vapor energy equation excluding the mole fraction derivatives [arbitrary dimensions]

$i \quad$ The imaginary urit such that $i^{2}=-1$.

i Specific enthalpy $[\mathrm{J} / \mathrm{kg}]$

$1 \quad$ integer value [dimensionless]

ICL Continuous liquid indicator, Eq. (47)

ILPC Collision-persisting model indicator (Sec. VI.B)

ImF Melting-freezing indicator, Eq. (119)

IT Topology indicator, Eq. (17)

Phase indicator, Eq. (44) 
IH Interfacial energy production over a time step [ $\mathrm{J} / \mathrm{kg}]$

IT FORTRAN iteration index [dimensionless]

$\vec{j}$ Molar diffusion flux relative to the average molar velocity [kmol/ $\left./ \mathrm{m}^{2}-\mathrm{s}\right]$

Ja Jacob number $\left(c_{p L} \Delta T_{\rho L}\right) /\left(h_{\ell g} \rho_{G}\right)$ [dimensionless]

$k \quad$ Thermal conductivity $[\mathrm{W} / \mathrm{m}-\mathrm{K}]$

$k$ Boltzmann constant [J/K-molecule]

$\vec{k} \quad$ Wave number $\left[\mathrm{m}^{-1}\right]$

K Interfield momentum-exchange coefficient $\left[\mathrm{N}-\mathrm{s} / \mathrm{m}^{4}\right]$

$L \quad$ Length $[\mathrm{m}]$

$m$ or $M$ The results of a mass surmation [kg]

M Molecular weight $[\mathrm{kg} / \mathrm{kmol}]$

n Number per unit volume $\left[\mathrm{m}^{-3}\right]$

$n^{\star} \quad$ Number per unit volume and unit time $\left[m^{-3} \mathrm{~s}^{-1}\right]$

N Integer variable [dimensionless]

$\mathrm{n}_{\mathrm{A}} \quad$ Number of gas atoms per molecule [dimensionless]

$\overrightarrow{\mathbf{N}}_{k} \quad$ Molar flux of species $k$ relative to a stationary coordinate reference frame $\left[\mathrm{kmol} / \mathrm{m}^{2}-\mathrm{s}\right]$

Nu Nusselt number $\left(d_{h} h / k\right.$ or $\left.2 r_{d} h / k\right)$ [dimensionless] 
o Outflow convectible area flux $\left[\mathrm{s}^{-1}\right]$

p Pressure $\left[\mathrm{N} / \mathrm{m}^{2}\right]$

Pcell Cell pressure $\left[\mathrm{N} / \mathrm{m}^{2}\right]$

$p_{G M}, p_{G m}$, Partial pressure of vapor material component $M$ (or $m$ ) $\left[N / m^{2}\right]$

or pgm ( $p G M$ preferred)

pM Input coefficient in the vapor pressure-temperature relationship for material $M\left[N / \mathrm{m}^{2}\right]$

$p^{\prime}, p^{+}, \quad$ Absolute value of pressure spatial gradients $\left[\mathrm{N} / \mathrm{m}^{3}\right]$

and $p^{\star}$

$p_{1 \phi} \quad$ Single-phase equilibrium pressure $\left[\mathrm{N} / \mathrm{m}^{2}\right]$

$P_{2 \phi} \quad$ Two-phase equilibrium pressure $\left[\mathrm{N} / \mathrm{m}^{2}\right]$

Pe Peclet number $\left(\operatorname{RePr}=D_{h}|\vec{v}| \rho c_{p} / k\right)$ [dimensionless]

$\operatorname{Pr}$ Prandt I number $\left(c_{p} \mu / k\right)$ [dimensionless]

Psf Single-phase component of the pressure in the AEOS option $\left[\mathrm{N} / \mathrm{m}^{2}\right]$

q Heat $f / u x\left[w / m^{2}\right]$

Q Volumetric energy source term $\left[w / m^{3}\right]$

$r$ Radius of bubble, droplet, or particle [in]

r Radial coordinate [m]

R Gas constant [J/kg-K] 
$\overrightarrow{\mathbf{R}} \quad$ Right-side vector used in obtaining derivatives of the velocities with respect to pressure $[\mathrm{s} / \mathrm{m}]$

Ro Universal gas constant $[\mathrm{J} / \mathrm{kmol}-\mathrm{K}]$

Re Reynolds number $\left(D_{h}|\vec{v}| \rho / \mu\right)$ [dimensionless]

$R_{r} \quad$ Intermediate term to maintain constant internal energies in Step 3 for energy component $r$ [K]

$R t_{r i}$ and Intermediate quantities for the Courant time-step control $\left[\mathrm{s}^{-1}\right]$ $\mathrm{Rt}_{\mathrm{zj}}$

s Specific entropy $[\mathrm{J} /(\mathrm{kg}-\mathrm{K})]$

$S \quad$ Sum of surface tensions $\left[\mathrm{kg} / \mathrm{s}^{2}\right]$

$S \quad$ Surface area source $\left[\mathrm{m}^{2} / \mathrm{m}^{3}-\mathrm{s}\right]$

$\overrightarrow{\mathbf{s}} \quad$ Vector containing the residual errors driving the pressure iteration [variable dimensions]

S Three-by-three coefficient matrix used in solving the momentum equations $\left[\mathrm{kg} / \mathrm{m}^{3}\right]$

$t \quad$ Time $[s]$

T Temperature [K]

$T_{M}^{\star} \quad$ Input exponent in the vapor pressure-temperature relationship for material $M[K]$

$T_{p}^{\prime} \quad$ Particle/vapor interface temperature with mass transfer absent [K] 
$T_{S}^{l} \quad$ Structure/vapor interface temperature with mass transfer absent [K]

u Radial velocity component $[\mathrm{m} / \mathrm{s}]$

$\vec{v} \quad$ velocity $[\mathrm{m} / \mathrm{s}]$

$\checkmark \quad$ Axial velocity component $[\mathrm{m} / \mathrm{s}]$

$\checkmark$ Representing either an axial or a radial velocity component [ $\mathrm{m} / \mathrm{s}$ ]

$\checkmark \quad$ Volume $\left[\mathrm{m}^{3}\right]$

$\mathrm{VC}_{G}$ Overall coefficient in the virtual mass equation $\left[\mathrm{kg} / \mathrm{m}^{3}\right]$

$\overrightarrow{\text { vin }} \quad$ Virtual mass term $\left[\mathrm{N} / \mathrm{m}^{3}\right]$

$V_{L 1, P}$ Volume around a particle affected by turbulence $\left[\mathrm{m}^{3}\right]$

$\mathrm{V}_{\mathrm{qq}}^{\star} \quad$ Velocity difference used in Step 2 for the turbulent interfield momentum coupl ing term $[\mathrm{m} / \mathrm{s}]$

$w_{m} \quad$ Mass fraction of gas material component $m$ [dimensionless]

Weighting factor [dimensionless]

W Pressure-volume or gravity work term $\left[\mathrm{J} / \mathrm{m}^{3}\right]$

I. Three-by-three coefficient matrix of energy derivatives with respect to temperature $[\mathrm{J} / \mathrm{kg}-\mathrm{K}]$

We Weber number $\left[\rho_{C P}\left(v_{C P}-v_{D}\right)^{2} d_{D} / \sigma_{D}, C P\right]$ [dimensionless]

$x \quad$ Vector component generally indicating a particular independent variable [variable dimensions] 
$x \quad$ Function argument [variable dimensions]

$x \quad(1 / 0)$ for values implying (nominal case/total vaporization) [dimensionless ]

$Y_{G m} \quad$ Mole fraction of vapor component $m$ [dimensioniess]

$z \quad$ Axial coordinate [m]

$\alpha \quad$ Volume fraction [dimensionless]

$\tilde{\alpha} \quad$ Minimum effective vapor volume fraction $\left[\tilde{\alpha}=\alpha_{0}\left(1-\alpha_{S}\right)\right]$ [dimensionless ]

$\alpha_{0} \quad$ Minimum input vapor volume fraction [dimensionless]

$\alpha_{G, \text { eff }}$ Effective gas volume fraction $\left[\alpha_{G, \text { eff }}=\alpha_{G}+\alpha_{0} \alpha_{L}\right.$ ] [dimensionless]

age Effective gas volume fraction for two-phase flow $\left[\alpha_{g e}=\max \right.$ $\left(\alpha_{G}, e f f, \tilde{\alpha}\right)$ ] [dimensionless]

$\alpha_{\text {Lo }}$ The total liquid volume fraction at the single-phase/two-phase transition [dimensionless]

$\alpha_{\text {mo }}$ Volume fraction for component in at the single-phase/two-phase transition [dimensionless]

${ }^{\alpha} \mathrm{S}, 10$ input volume fraction for infinite (large) structure drag [dimensionless ]

$\beta \quad$ Angle [dimensionless]

$\gamma \quad$ Heat capacity ratio [dimensionless] 
I Mass-transfer rate per unit volume. When subscripted, the positive rate is from the component corresponding to the first subscript to the component representing the second subscript $\left[\mathrm{kg} / \mathrm{m}^{3}-\mathrm{s}\right]$

$\mathrm{r}_{\mathrm{C}} \quad$ Coalescence rate $[\mathrm{m} / \mathrm{s}]$

$\delta \quad$ Boundary-layer thickness [m]

$\delta \quad$ Minimum stable film of vapor on a liquid 2 (coolant) droplet [m]

$\delta \quad$ Variation of a physical quantity [dimensionless]

$\delta_{i j}$ or Kronecker delta [dimensionless]

$\delta(i, j)$

$\Delta \quad$ Difference [dimensionless]

$\Delta r \quad$ Radial mesh spacing [m]

st Time-step size [s]

$\Delta T_{h} \quad$ Temperature range over which the homogeneous condensation term is quadratic in the temperature difference [K]

$\Delta \mathrm{T}$ Sup Superheat $[\mathrm{K}]$

$\Delta z \quad$ Axial mesh spacing $[\mathrm{m}]$

e Turbulent dissipation rate $\left[\mathrm{m}^{2} / \mathrm{s}^{3}\right]$

E Emissivity [dimensionless]

E Error in the cell pressure as compared with the EOS $\left[\mathrm{N} / \mathrm{m}^{2}\right]$ 
$\varepsilon \quad$ Characteristic energy of interaction between molecules (LennardJones parameter) [J]

$\varepsilon_{p} \quad$ Convergence criterion for the pressure calculation in the SESAME interface $\left[\mathrm{N} / \mathrm{m}^{2}\right]$

$\varepsilon_{v} \quad$ Convergence criterion used in solving the momentum equations [dimensionless]

$\varepsilon_{T} \quad$ Convergence criterion for the temperature calculation in the SESAME interface [dimensionless]

$\zeta \quad$ Thermal diffusivity $\left[\mathrm{m}^{2} / \mathrm{s}\right]$

$\theta \quad$ Nondimensional temperature [dimensionless]

$\lambda \quad$ Virtual mass parameter based on Drew's objectivity principle [dimensionless]

$\lambda \quad$ Matrix eigenvalue $\left[w / m^{3}-k\right]$

$\lambda \quad$ Wavelength $[\mathrm{m}]$

$\lambda_{C} \quad$ Critical wavelength $[\mathrm{m}]$

A Axial/radial discriminator being (1/0) for the (axial/radial) direction

$\Lambda$ Derivative in the Taylor expansion of the mass-transfer rates [variable dimensions]

$\Lambda_{\text {rq }}$ Intermediate matrix used to maintain constant internal energies in Step 3 for energy component $r$ and momentum field $q\left[\mathrm{~K}-\mathrm{m}^{3} / \mathrm{kg}\right]$

H Viscosity (dynamic) $[\mathrm{kg} / \mathrm{m}-\mathrm{s}]$ 
$\mu \quad$ Chemical potential $[\mathrm{J} / \mathrm{kmol}]$

$v \quad$ Viscosity (kinematic) $\left[\mathrm{m}^{2} / \mathrm{s}\right]$

$\xi \quad$ Surface area multiplier [dimensionless]

$\xi \quad$ Area coordinate, $\pi r^{2}\left[\mathrm{~m}^{2}\right]$

$\xi_{d} \quad$ Input interfield momentum-exchange function multiplier [dimensionless]

$\pi \quad$ Pi (3.1415926536 ... )

P Microscopic density $\left[\mathrm{kg} / \mathrm{m}^{3}\right]$

$\bar{\rho} \quad \alpha \rho$, Macroscopic (smear) density $\left[\mathrm{kg} / \mathrm{m}^{3}\right]$

$\rho_{L, e f f}$ Virtual (effective) density $\left[\mathrm{kg} / \mathrm{m}^{3}\right]$

Plmo or Microscopic density at the single-phase/two-phase transition PLmo $\left[\mathrm{kg} / \mathrm{m}^{3}\right]$

- Surface tension $\left[\mathrm{kg} / \mathrm{s}^{2}\right]$

- Stefan-Boltzmann constant $\left[W / m^{2}-k^{4}\right]$

$\sigma \quad$ Molecular diameter (Lennard-Jones parameter) [A]

oLG Surface tension between I iquid and gas $\left[\mathrm{kg} / \mathrm{s}^{2}\right]$

oL Surface tension between two liquids $\left[\mathrm{kg} / \mathrm{s}^{2}\right]$

$\tau$ Time constant [s]

\$ Heat flux $\left[\mathrm{W} / \mathrm{m}^{2}\right]$ 
* Flow specifier [dimensionless]

* Arbitrary function [arbitrary dimensions]

$\omega \quad$ Probability [dimensionless]

$\omega \quad$ Time-mixing parameter [dimensionless]

$\Omega_{\mu} \quad$ Collision integral for viscosity. [dimensionless]

SD Collision integral for mass diffusivity [dimensionless]

< Brackets to indicate a quantity evaluated for spatial differencing [dimensionless]

\section{Definition}

a Average or mixture

basic Unmodified interfield momentum-exchange coefficients

b Bubble

BO Burnout

BFR Bubbly flow regime

C or c Continuous (no preference)

CC Liquid-continuous churn flow

$C D$ or cd Continuous-discontinuous (no preference)

$\mathrm{CL}$ Continuous liquid 
CLDL Between the continuous and discontinuous I iquids

CM Cormon for the liquid 1 solid particulate system

Con Refers to condensate conditions at the local partial pressure of the vapor

$\mathrm{CP} \quad$ Cont inuous phase

CS Continuous structure

Crt, Crit, Critical (Crt preferred)

or crit

D or d Discontinuous sphere or droplet

dc Direct contact

Do Discont inuous-discont inuous

DL Discontinuous liquid

DP Discontinuous phase

DS Discontinuous structure

e Equilibrium

e Internal energy

EC Effective instantaneous contact

ECS Equivalent common surface

eff Effective 
ext External source

F Fragmentation

FB Film boiling

g,v Gravity and velocity

9 A vapor property

G Vapor energy or vapor momentum field

GCL Between vapor and the cont inuous phase

GOL Between vapor and the discont inuous phase

A comma is used to separate two energy component subscripts, whereas a slash is used to separate two momentum component subscripts. If no danger of confusion exists, the comma or slash may be omitted. When possible, omission of the separator is the preferred usage for reasons of economy.

G, Lm or GLm Between vapor and I iquid energy component $m$

G/Lm or GLm Between vapor and liquid momentum component $m$

gm Vapor density component m

Gm or GN Vapor material component m (Gm preferred)

G,P or GP Between vapor and the particle component

G,S or GS Between vapor and the structure energy components

G/S or GS Between the vapor and structure momentum components 
H Heat transfer

i Arbitrary species component

i Energy component subscript

i Radial spatial index

i Interface subscript

IC Instantaneous contact

j Matrix index

j Axial spatial index

k A second density, energy, or material component

L Liquid (generally referring to a mixture of all liquids present)

LE Leidenfrost

Liq Liquidus

em Liquid density component $m$

Lm Liquid energy/material $m$ or liquid momentum field $\mathrm{m}$

Lm, $G$ or LmG Between I iquid energy component $m$ and vapor

$L m / G$ or $L m G$ Between liquid momentum field $m$ and vapor

Lm, Lm' or LmLm' Between liquid energy field Lm and Lm'

Lm, $P$ or LmP Between liquid energy component $m$ and structure 
Lm, $S$ or LmS Between liquid energy component $m$ and structure

$\mathrm{Lm} / \mathrm{S}$ or $\mathrm{LmS}$ Between liquid momentum component $m$ and structure

LM Liquid mixture

LL Nonvapor flow

L1P Between liquid 1 and particulates

L1CP Between liquid 1 and the cont irruous phase

$m$ When used alone, $m$ refers to a density component or a summation index. In combination with other subscripts or independently defined symbols it also can refer to a energy or material component. [In the SESAME/AFDM interface theory, " $m$ " refers to the particle and I iquid components.]

M Material component

max Maximum

mb Minimum bubble

mo Minimum droplet

Min or min Minimum

MIt or melt Melting (MIt preferred)

MF Melting-freezing

$m \times b \quad$ Maximum bubble

mxd Maximum droplet 
n Vapor components in the SESAME/AFDM interface theory

Noncoalescing

- The single-phase/two-phase transition point

p Particle density component

p Pressure

P Particle energy or density component

PCP Between solid particulate and the continuous phase

$P, G$ or $P G$ Between the particle and the vapor energy components

P,Lm or PLm Between the particles and the energy component for liquid $m$

P,S or PS Between the particle and structure components

9 Arbitrary velocity component

$q^{\prime} \quad$ A second velocity component

L1, L2, and $G$ normally are used to identify momentum fields. However, when a confusion with energy components is possible, the following notation is used.

$q=L_{1}$ or $q^{1} \quad$ Signifying momentum field 1

$q=L 2$ or $q 2 \quad$ Signifying momentum field 2

q3 Signifying momentum field 3

$r \quad$ Energy component 
S Structure density component

S Spinodal value (see Spg and SpI)

S Structure energy or density component

Sat or sat Saturation based on vapor partial pressure [Sat, Gm indicates saturation conditions for vapor component $m$ ] (Sat is preferred)

S,G or SG Between the structure and vapor energy components

S/G or SG Between the structure and vapor momentum components

S,Lm or SLm Between the structure and liquid energy component $m$

$\mathrm{S} / \mathrm{Lm}$ or SLm Between the structure and I iquid momentum component $m$

SId Referring to values of pressure and internal energy from the solid EOS table

Sol Solidus

S,P or SP Between the structure and particle components

Spg Vapor spinodal limit

Spl Liquid spinodal limit

SS Solid structure

Sub Sublimation

Sup or sup Superheat (Sup preferred)

Sus Solid side of the sublimation curve 
T Topology

$T=0 \quad$ Signifies absolute zero

TR Threshold of levitation

TZ Hysteresis transition

$\vec{v} \quad$ Velocity

Van Referring to values of pressure and internal energy from the SESAME 301 table

Vap Referring to saturated vapor conditions at the local partial pressure of the vapor

x A component subscript defined based on local needs

$X, Y$ Between $X$ and $Y$

$\theta$ or $\theta+$ Spatial index, $(i, j+1 / 2)(\theta+i$ s to be used when $\theta-i$ possible $)$

$\theta-\quad$ Spatial index, $(i, j-4 / 2)$

$\lambda$ or $\lambda+$ Spatial index, $(i+3 / 2, j)(\lambda+i$ s to be used when $\lambda-i$ s possible)

$\lambda-\quad$ Spatial index, $(i-1 / 2, j)$

$\xi \quad$ Spatial index representing either $\lambda$ or $\theta$

p Thermodynamic density

Phase 


\section{Dofinition}
a Associated
c Convection
FB Film boiling
i Input value
I Interface
k Iteration index

mol Molar quantity

n Beginning-of-time-step value

$n+1$ End-of-time-step value

P Combination of persisting and collision contact

$\checkmark \quad$ Collision contact

* Iteration index

p Per unit mass

o Persisting contact

* Single-phase pressure equilibrium condition (used for the SESAME EOS interface)

t) Gradients or derivatives 


\section{Modifier Definition}

- Indicating a ce!l-averaged value

$\sim \quad$ Intermediate value

$\approx \quad$ Secondary intermediate value

- Vector

$\Lambda \quad$ Secondary (internal) index

\section{REFEREICES}

1. G. B. Wallis, One-Dimensional Two-Phase Flow, McGraw-Hill, New York (1969).

2. T. Ginsburg, O. C. Jones, C. E. Schwarz, and J. C. Chen, "Observations of Flow Characteristics of Volume-Heated Boiling Pools," Brookhaven National Laboratory report BNL-NNREG-24270 (1977).

3. Y. Taitel, D. Barnea, and A. E. Dukler, "Modelling Flow Pattern Transitions for Steady Upward Gas-Liquid Flow in Vertical Tubes," AlChE Journal, Vol. 26, No. 3, pp. 345-354 (1980).

4. M. Ishi i and K. Mishima, "Study of Two-Fluid Model and Interfacial Area," Argonne National Laboratory report NUREG/CR-1873 (1980).

5. Y. Taitel and D. Barnea, "Counter Current Gas-Liquid Vertical Flow, Model for Flow Pattern and Pressure Drop," Int. J. Multiphase Flow, Vol. 9, №. 6, pp. 637-647 (1983).

6. R. C. Darton and D. Harrison, "Gas and Liquid Hold-Up in Three-Phase Fluidisation," Chem. Engineering Science, Vol. 30, pp. 581-586 (1975).

7. N. Abuaf, B. J. C. Wu, G. A. Zimmer, and P. Saha, "A Study of Nonequilibrium Flashing of Water in a Converging-Diverging Nozzle," Brookhaven National Laboratory report BNL-NUREG-51317, NUREG/CR-1864, Vol. 2 (1980).

8. G. Kocarnustafaogullari, I. Y. Chen and M. Ishii, "Unified Theory for Predicting Maximum Fluid Particle Size for Drops and Bubbles," Argonne National Laboratory report ANL-84-67, NUREG/CR-4028 (1984). 
9. S. A. Krzeczkowski, "Measurement of Liquid Droplet Disintegration Mechanism," Int. J. Multiphase Flow, Vol. 6, pp. 227-239 (1980).

10. M. Pilch and C. A. Erdman, "Use of Breakup Time Data and Velocity History Data to Predict the Maximum Size of Stable Fragments for

Acceleration-Induced Breakup of a Liquid Drop," Int. J. Multiphase Flow, Vol. 13, No. 6, pp. 741-757 (1987).

11. M. Sevik and S. H. Park, "The Splitting of Drops and Bubbles by Turbulent Fluid Flow," J. Fluids Engng., Vol. 95, pp. 53-60 (1973).

12. J. 0. Hinze, "Fundamental of Splitting in Dispersion Processes," AlChE Journal, Vol. 1, pp. 289-295 (1955).

13. T. G. Theofanous and J. Sullivan "Turbulence in Two-Phase Dispersed Flows," J. Fluid Mech., Vol. 116, pp. 343-362 (1982).

14. A. Koestel, R. G. Gido, and D. E. Lamkin, "Drop-Size Estimates for a Loss-of-Coolant-Accident," Los Alamos National Laboratory report LA-8449-MS, NUREG/CR-1607 (1980).

15. L. L. Smith, "SIMMER-11: A Computer Program for LMFBR Disrupted Core Analysis," Los Alamos National Laboratory report LA-7515, NUREG/CR-0453, Rev. (1980).

16. Y. H. Mori, "Configurations of Gas-Liquid Two-Phase Bubbles in Immiscible Liquid Media," Int. J. Multiphase Flow, Vol. 4, pp. 383-396 (1978).

17. S. Kondo, PNC, 0-arai, Japan, private communication, February 29, 1988. 